\title{
Ligand-Controlled Regiodivergent Hydroformylation of Ynamides: A Stereospecific and Regioselective Access to 2- and 3-Aminoacroleins
}

Patrick Wagner, ${ }^{\dagger}$ Morgan Donnard, ${ }^{* *}$ Nicolas Girard ${ }^{\dagger *}$

$\dagger$ Université de Strasbourg, CNRS (LIT UMR 7200); Faculté de Pharmacie; F-67000 Strasbourg * Université de Strasbourg, CNRS, Université de Haute-Alsace (LIMA UMR 7042); École Européenne de

Chimie, Polymères et Matériaux (ECPM); F-67000 Strasbourg

Corresponding Authors:

* E-mail: donnard@unistra.fr

* E-mail: nicolas.girard@unistra.fr

\section{Supporting information}

Table of contents

1. General information

S2

2. Experimental procedures

S2

2.1. Synthesis of ynamides $1 \mathbf{a - q}$

$\mathrm{S} 2-\mathrm{S} 7$

2.2. Hydroformylations of ynamides

$\mathrm{S} 8-\mathrm{S} 16$

3. ${ }^{1} \mathrm{H} \&{ }^{13} \mathrm{C}$ NMR spectra

S17-S101 


\section{General information}

All reagents, chemicals, ligands (except BiPhePhos) and dry solvents were purchased from commercial sources and used without purification. (Acetylacetonato)dicarbonylrhodium(I) was purchased from Sigma-Aldrich Co. BiPhePhos was prepared as reported previously by Buchwald et al.. ${ }^{1}$ All experiments were performed under argon atmosphere except where otherwise noted. All hydroformylation reactions were performed in a high pressure reactor from Parr Instrument Company using gases supplied by Air Liquide. Reactions were monitored by TLC (Thin Layer silica gel Chromatography) using Merck silica gel $60 \mathrm{~F}_{254}$ on aluminium sheets. TLC plates were visualized under UV light and revealed with acidic $p$-anisaldehyde stain or $\mathrm{KMnO}_{4}$ stain. Crude products were purified by flash column chromatography on Merck silica gel Si 60 (40-63 $\mu \mathrm{m}$ ) or by using CombiFlashRf (Teledyne Isco). ${ }^{1_{\mathrm{H}}}$ and ${ }^{13} \mathrm{C}$ NMR spectra were recorded on a Bruker $(500 \mathrm{MHz} / 125 \mathrm{MHz}$ and $400 \mathrm{MHz} / 100 \mathrm{MHz}$ ) spectrometer. Conditions are specified for each spectrum (temperature $25{ }^{\circ} \mathrm{C}$ unless specified). Chemical shifts are reported in parts per million (ppm) relative to residual solvent and coupling constants $(J)$ are reported in hertz $(\mathrm{Hz})$. Signals are described as s (singlet), d (doublet), t (triplet), q (quartet), m (multiplet), dd (doublet of doublets), dt (doublet of triplets), ddt (doublet of doublet of triplets), br s (broad singlet), br d (broad doublet), br q (broad quadruplet) and br dd (broad doublet of doublets). Deutered solvents were purchased from Eurisotop $^{\circledR}$. HRMS were obtained on an Agilent Technologie 6520 Accurate-Mass Q.Tof LC/MC apparatus using electrospray ionization mode and time-of flight analyzer (ESI-TOF).

\section{Experimental procedures}

\subsection{General procedures for the synthesis of ynamides 1a-n}

Under argon in an oven-dried flask, carbamate or sullfonamide (1 equiv.), $\mathrm{CuSO}_{4} .5 \mathrm{H}_{2} \mathrm{O}(0.2$ equiv.), 1,10phenanthroline or $(1 R, 2 R)-N, N^{\prime}$-dimethyl-1,2-cyclohexanediamine ( 0.4 equiv.) and $\mathrm{K}_{3} \mathrm{PO}_{4}$ ( 2 equiv.) was added to a solution of alkynyl bromide (1.1 - 1.5 equiv.) in anhydrous toluene (0.8 to $1.0 \mathrm{M}$ ). The flask was backfilled 3 times with argon and stirred at $70^{\circ} \mathrm{C}$ except $1 \mathbf{1 f}\left(110^{\circ} \mathrm{C}\right)$ for $16 \mathrm{~h}$ except for $\mathbf{1 f}, \mathbf{1 g}, \mathbf{1 n}(72 \mathrm{~h})$. The mixture was diluted with chloroform, filtered through celite pad, washed with methylene chloride and concentrated under vacuum. The crude product was purified by flash column chromatography using a heptane / ethyl acetate mixture as eluant.

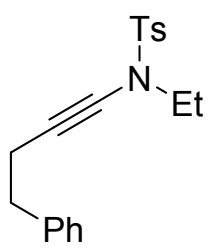

N-ethyl-4-methyl-N-(4-phenylbut-1-yn-1-yl)benzene-1-sulfonamide (1a)

Synthesized following the general procedure 2.1 using $N$-ethyl-4-methylbenzene-1-sulfonamide (900 mg, 4.52 mmol), (4-bromobut-3-yn-1-yl)benzene, 1,10-phenanthroline as ligand, and heptane/EtOAc (85:15) as chromatography eluent. Entitled product 1a was obtained in $85 \%$ yield (1.25 g) as pale yellow oil.

${ }^{1} \mathrm{H}$ NMR $(400 \mathrm{MHz}$, Chloroform- $d$ ) $\delta=1.11(\mathrm{t}, J=7.2 \mathrm{~Hz}, 3 \mathrm{H}), 2.44(\mathrm{~s}, 3 \mathrm{H}), 2.58(\mathrm{t}, J=7.3 \mathrm{~Hz}, 2 \mathrm{H}), 2.81(\mathrm{t}, J=7.3 \mathrm{~Hz}$, $2 \mathrm{H}), 3.30(\mathrm{q}, J=7.2 \mathrm{~Hz}, 2 \mathrm{H}), 7.18-7.22(\mathrm{~m}, 3 \mathrm{H}), 7.28-7.30(\mathrm{~m}, 4 \mathrm{H}), 7.70(\mathrm{~d}, J=8.0 \mathrm{~Hz}, 2 \mathrm{H}) ;{ }^{13} \mathrm{C} \mathrm{NMR}(101 \mathrm{MHz}$, Chloroform-d) $\delta=13.2,20.8,21.8,35.4,46.7,69.7,73.6,126.4,127.7,128.5,128.6,129.7,135.0,140.7,144.3$; HRMS (ESI) : calculated for: $\mathrm{C}_{19} \mathrm{H}_{22} \mathrm{NO}_{2} \mathrm{~S}[\mathrm{M}+\mathrm{H}]^{+}: 328,1366$, found 328.1359 .

\footnotetext{
${ }^{1}$ Cuny, G. D.; Buchwald, S. L. J. Am. Chem. Soc. 1993, 115, 2066.
} 


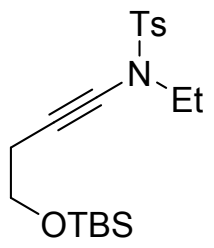

N-\{4-[(tert-butyldimethylsilyl)oxy]but-1-yn-1-yl\}-N-ethyl-4-methylbenzene-1-sulfonamide (1b)

Synthesized following the general procedure 2.1 using $N$-ethyl-4-methylbenzene-1-sulfonamide (199 mg, 1.00 mmol), [(4-bromobut-3-yn-1-yl)oxy](tert-butyl)dimethylsilane, 1,10-phenanthroline as ligand, and heptane/EtOAc (90:10) as chromatography eluent. Entitled product $\mathbf{1 b}$ was obtained in $78 \%$ yield (299 $\mathrm{mg})$ as colorless oil.

${ }^{1} \mathrm{H}$ NMR (400 MHz, Chloroform-d) $\delta=0.05(\mathrm{~s}, 6 \mathrm{H}), 0.88(\mathrm{~s}, 9 \mathrm{H}), 1.18(\mathrm{t}, \mathrm{J}=7.2 \mathrm{~Hz}, 3 \mathrm{H}), 2.44(\mathrm{~s}, 3 \mathrm{H}), 2.48(\mathrm{t}, \mathrm{J=7.2}$ $\mathrm{Hz}, 2 \mathrm{H}), 3.34(\mathrm{q}, J=7.2 \mathrm{~Hz}, 2 \mathrm{H}), 3.67(\mathrm{t}, J=7.2 \mathrm{~Hz}, 2 \mathrm{H}), 7.31-7.34(\mathrm{~m}, 2 \mathrm{H}), 7.76-7.79(\mathrm{~m}, 2 \mathrm{H}) ;{ }^{13} \mathrm{C} \mathrm{NMR}(101 \mathrm{MHz}$, Chloroform-d) $\delta=-5.3,13.2,18.3,21.6,22.9,25.9,46.5,62.2,67.4,73.7,127.6,129.6,134.8,144.3$; HRMS (ESI) : calculated for: $\mathrm{C}_{19} \mathrm{H}_{31} \mathrm{NO}_{3} \mathrm{SSi}[\mathrm{M}+\mathrm{H}]^{+}: 382,1867$, found 382.1873 .<smiles>CCN([12F])C#Cc1ccccc1</smiles>

$\mathrm{N}$-ethyl-4-methyl-N-(2-phenylethynyl)benzene-1-sulfonamide (1c) ${ }^{2}$

Synthesized following the general procedure 2.1 using $N$-ethyl-4-methylbenzene-1-sulfonamide $(200 \mathrm{mg}, 1.00$ mmol), (2-bromoethynyl)benzene, ( $1 R, 2 R)-N, N^{\prime}$-dimethyl-1,2-cyclohexanediamine as ligand, and heptane/EtOAc (90:10) as chromatography eluent. Entitled product $1 \mathrm{c}$ was obtained in $90 \%$ yield $(269 \mathrm{mg})$ as colorless oil.

${ }^{1} \mathrm{H}$ NMR (500 MHz, Chloroform-d) $\delta=1.28(\mathrm{t}, J=7.2 \mathrm{~Hz}, 3 \mathrm{H}), 2.45(\mathrm{~s}, 3 \mathrm{H}), 3.49$ (q, J=7.2 Hz, 2H), $7.27-7.31$ (m, 3H), $7.35-7.38(\mathrm{~m}, 4 \mathrm{H}), 7.83-7.85(\mathrm{~m}, 2 \mathrm{H}) ;{ }^{13} \mathrm{C}$ NMR $\left(126 \mathrm{MHz}, \mathrm{CDCl}_{3}\right) \delta=13.4,21.8,46.9,70.9,82.2,123.0$, $127.8,127.9,128.4,129.9,131.5,134.8,144.7$.<smiles>CCN(C)C#CS(C)(=O)=O</smiles>

\section{$\mathrm{N}$-ethyl-4-methyl-N-[2-(trimethylsilyl)ethynyl]benzene-1-sulfonamide (1d)}

Synthesized following the general procedure 2.1 using $N$-ethyl-4-methylbenzene-1-sulfonamide $(400 \mathrm{mg}, 2.01$ mmol), (2-bromoethynyl)trimethylsilane, $(1 R, 2 R)-N, N^{\prime}$-dimethyl-1,2-cyclohexanediamine as ligand, and heptane/EtOAc (90:10) as chromatography eluent. Entitled product $1 \mathrm{~d}$ was obtained in $29 \%$ yield $(174 \mathrm{mg})$ as colorless oil.

${ }^{1} \mathrm{H}$ NMR (400 MHz, Chloroform-d) $\delta=0.16(\mathrm{~s}, 9 \mathrm{H}), 1.20(\mathrm{t}, J=7.2 \mathrm{~Hz}, 3 \mathrm{H}), 2.45(\mathrm{~s}, 3 \mathrm{H}), 3.38$ (q, J=7.2 Hz, 2H), 7.32 $-7.35(\mathrm{~m}, 2 \mathrm{H}), 7.78-7.80(\mathrm{~m}, 2 \mathrm{H}) ;{ }^{13} \mathrm{C}$ NMR $\left(101 \mathrm{MHz}, \mathrm{CDCl}_{3}\right) \delta=0.3,13.2,21.8,46.6,73.5,94.9,127.9,129.7$, 134.9, 144.7 ; HRMS (ESI) : calculated for: $\mathrm{C}_{14} \mathrm{H}_{22} \mathrm{NO}_{2} \mathrm{SSI}[\mathrm{M}+\mathrm{H}]^{+}: 296,1135$, found 296.1136.<smiles>[3H]N(C#C)CC</smiles>

\section{$\mathrm{N}$-ethyl-N-ethynyl-4-methylbenzene-1-sulfonamide (1e) ${ }^{2}$}

Synthesized following the general procedure 2.1 using $N$-ethyl-4-methylbenzene-1-sulfonamide $(400 \mathrm{mg}, 2.01$ mmol), (2-bromoethynyl)tris(propan-2-yl)silane and 1,10-phenanthroline as ligand. Entitled product 1e was

\footnotetext{
2 Tu, Y.; Zheng, X.; Wang, H.; Zhao, J. Org. Lett. 2018, 20, 280.
} 
obtained after a deprotection procedure using TBAF (1.0 M in THF) at $-5{ }^{\circ} \mathrm{C}$ and a purification by flash column chromatography using heptane/EtOAc (90:10) as eluent, to give a yellow oil in $70 \%$ yield (138 mg).

${ }^{1} \mathrm{H}$ NMR (400 MHz, Chloroform-d) $\delta=1.20(\mathrm{t}, J=7.2 \mathrm{~Hz}, 3 \mathrm{H}), 2.44(\mathrm{~s}, 3 \mathrm{H}), 2.73(\mathrm{~s}, 1 \mathrm{H}), 3.38(\mathrm{q}, \mathrm{J}=7.2 \mathrm{~Hz}, 2 \mathrm{H}), 7.32$ - $7.36(\mathrm{~m}, 2 \mathrm{H}), 7.78-7.81(\mathrm{~m}, 2 \mathrm{H}) ;{ }^{13} \mathrm{C}$ NMR (101 MHz, Chloroform-d) $\delta=13.1,21.7,46.5,59.3,75.8,127.7$, $129.9,134.9,144.8$.

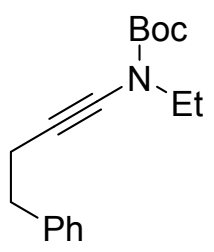

tert-butyl $\mathbf{N}$-ethyl-N-(4-phenylbut-1-yn-1-yl)carbamate (1f)

Synthesized following the general procedure 2.1 using tert-butyl $N$-ethylcarbamate $(127 \mathrm{mg}, 0.88 \mathrm{mmol}$ ), (4bromobut-3-yn-1-yl)benzene, 1,10-phenanthroline as ligand, reaction time $72 \mathrm{~h}$, heating $110{ }^{\circ} \mathrm{C}$, and heptane/EtOAc (98:2) as chromatography eluent. Entitled product 1f was obtained in $29 \%$ yield (70 mg) as colorless oil.

${ }^{1} \mathrm{H}$ NMR (400 MHz, Chloroform- $d$ ) $\delta=1.16(\mathrm{t}, J=7.2 \mathrm{~Hz}, 3 \mathrm{H}), 1.48(\mathrm{~s}, 9 \mathrm{H}), 2.60(\mathrm{t}, J=7.5 \mathrm{~Hz}, 2 \mathrm{H}), 2.84(\mathrm{t}, J=7.5 \mathrm{~Hz}$, $2 \mathrm{H}), 3.39$ (q, J=7.2 Hz, 2H), $7.18-7.31(\mathrm{~m}, 6 \mathrm{H}) .{ }^{13} \mathrm{C}$ NMR (126 MHz, Chloroform-d) $\delta=13.0,20.8,26.9,28.1,35.3$, 35.6, 44.3, 68.5, 74.9, 81.8, 82.6, 125.8, 126.2, 128.3, 128.5, 141.0, 142.0, 153.2, 154.5, 175.5; HRMS (ESI): calculated for: $\mathrm{C}_{17} \mathrm{H}_{23} \mathrm{NNaO}_{2}[\mathrm{M}+\mathrm{Na}]^{+}: 296,1621$, found 296.1622 .<smiles>CCN(C#CCCc1ccccc1)C(=O)OC</smiles>

methyl $\mathbf{N}$-ethyl-N-(4-phenylbut-1-yn-1-yl)carbamate (1g)

Synthesized following the general procedure 2.1 using methyl $N$-ethylcarbamate $(206 \mathrm{mg}, 2.00 \mathrm{mmol}$ ), (4bromobut-3-yn-1-yl)benzene, 1,10-phenanthroline as ligand, reaction time $72 \mathrm{~h}$, and heptane/EtOAc (95:5 to 90:10) as chromatography eluent. Entitled product $1 \mathrm{~h}$ was obtained in $46 \%$ yield $(213 \mathrm{mg})$ as colorless oil.

${ }^{1} \mathrm{H}$ NMR (400 MHz, Chloroform- $d$ ) $\delta=1.16(\mathrm{t}, J=7.2 \mathrm{~Hz}, 3 \mathrm{H}), 2.61(\mathrm{td}, J=7.4,0.6 \mathrm{~Hz}, 2 \mathrm{H}), 2.85(\mathrm{t}, J=7.4 \mathrm{~Hz}, 2 \mathrm{H}), 3.45$ (q, J=7.2 Hz, 2H), 3.78 (s, 3H), $7.19-7.31$ (m, 6H); ${ }^{13} \mathrm{C}$ NMR (126 MHz, Chloroform-d) $\delta=12.9,20.7,35.5,45.1$, 53.7, 69.2, 74.2, 126.2, 128.3, 128.5, 140.8, 156.0; HRMS (ESI): calculated for: $\mathrm{C}_{14} \mathrm{H}_{18} \mathrm{NO}_{2}[\mathrm{M}+\mathrm{H}]^{+}: 232,1332$, found 232.1327 .<smiles>O=C1OCCN1C#CCCc1ccccc1</smiles>

3-(4-phenylbut-1-yn-1-yl)-1,3-oxazolidin-2-one (1h) ${ }^{3}$

Synthesized following the general procedure 2.1 using 2-oxazolidone (131 mg, $1.50 \mathrm{mmol}$ ), (4-bromobut-3-yn-1yl)benzene, $(1 R, 2 R)-N, N^{\prime}$-dimethyl-1,2-cyclohexanediamine as ligand, and heptane/EtOAc (70:30) as chromatography eluent. Entitled product $1 \mathrm{~h}$ was obtained in $78 \%$ yield ( $253 \mathrm{mg}$ ) as colorless oil. ${ }^{1} \mathrm{H}$ NMR $(400 \mathrm{MHz}$, Chloroform- $d) \delta=2.58-2.62(\mathrm{~m}, 2 \mathrm{H}), 2.85(\mathrm{t}, \mathrm{J}=7.6 \mathrm{~Hz}, 2 \mathrm{H}), 3.81-3.85(\mathrm{~m}, 2 \mathrm{H}), 4.38-4.42$ $(\mathrm{m}, 2 \mathrm{H}), 7.21-7.23(\mathrm{~m}, 3 \mathrm{H}), 7.28-7.32(\mathrm{~m}, 2 \mathrm{H})$.

\footnotetext{
3 Jouvin, K.; Couty, F.; Evano, G. Org. Lett. 2010, 12, 3272.
} 


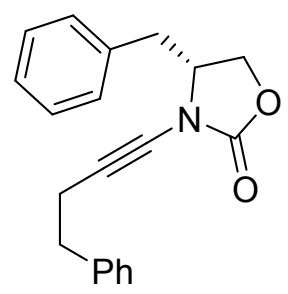

(4R)-4-benzyl-3-(4-phenylbut-1-yn-1-yl)-1,3-oxazolidin-2-one (1i) ${ }^{4}$

Synthesized following the general procedure 2.1 using $(R)$-4-benzyl-2-oxazolidinone (354 $\mathrm{mg}, 2.00 \mathrm{mmol}$ ), (4bromobut-3-yn-1-yl)benzene, $(1 R, 2 R)-N, N^{\prime}$-dimethyl-1,2-cyclohexanediamine as ligand, and heptane/EtOAc (80:20) as chromatography eluent. Entitled product $1 \mathbf{i}$ was obtained in $73 \%$ yield $(443 \mathrm{mg})$ as pale yellow oil.

${ }^{1} \mathrm{H}$ NMR (400 MHz, Chloroform- $d$ ) $\delta=2.68(\mathrm{t}, J=7.4 \mathrm{~Hz}, 2 \mathrm{H}), 2.80$ (dd, J=13.9, 8.3 Hz, 1H), 2.88 (t, J=7.4 Hz, 2H), 3.07 (dd, J=13.9, $3.9 \mathrm{~Hz}, 1 \mathrm{H}), 4.07$ (dd, J=8.4, $5.9 \mathrm{~Hz}, 1 \mathrm{H}), 4.10-4.17(\mathrm{~m}, 1 \mathrm{H}), 4.27$ (t, J=8.1 Hz, 1H), $7.13-7.15$ $(\mathrm{m}, 2 \mathrm{H}), 7.18-7.22(\mathrm{~m}, 1 \mathrm{H}), 7.24-7.26(\mathrm{~m}, 2 \mathrm{H}), 7.28-7.35(\mathrm{~m}, 5 \mathrm{H}) ;[\propto]_{D}^{18}=-116.0(c 2.00, \mathrm{DMF})$.

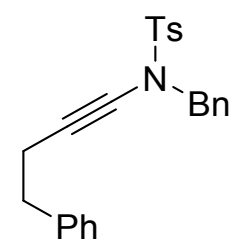

N-benzyl-4-methyl-N-(4-phenylbut-1-yn-1-yl)benzenesulfonamide (1j) ${ }^{5}$

Synthesized following the general procedure $\mathbf{2 . 1}$ using $N$-benzyl-4-methylbenzene-1-sulfonamide $(200 \mathrm{mg}, 0.77$ mmol), (4-bromobut-3-yn-1-yl)benzene, $(1 R, 2 R)-N, N^{\prime}$-dimethyl-1,2-cyclohexanediamine as ligand, and heptane/EtOAc (85:15) as chromatography eluent. Entitled product 1i was obtained in $86 \%$ yield $(256 \mathrm{mg})$ as colorless oil.

${ }^{1} \mathrm{H}$ NMR $(500 \mathrm{MHz}$, Chloroform-d) $\delta=2.44(\mathrm{~s}, 3 \mathrm{H}), 2.48(\mathrm{t}, \mathrm{J}=7.3 \mathrm{~Hz}, 2 \mathrm{H}), 2.69(\mathrm{t}, \mathrm{J}=7.3 \mathrm{~Hz}, 2 \mathrm{H}), 4.40$ (s, 2H), 7.08$7.10(\mathrm{~m}, 1.7 \mathrm{~Hz}, 2 \mathrm{H}), 7.18-7.21(\mathrm{~m}, 3 \mathrm{H}), 7.23-7.26(\mathrm{~m}, 3 \mathrm{H}), 7.27-7.29(\mathrm{~m}, 4 \mathrm{H}), 7.64-7.66(\mathrm{~m}, 2 \mathrm{H})$.<smiles>[Al-]N(C#CCCc1ccccc1)c1ccccc1</smiles>

4-methyl- $N$-phenyl- $N$-(4-phenylbut-1-yn-1-yl)benzenesulfonamide (1k) ${ }^{6}$

Synthesized following the general procedure 2.1 using 4-methyl- $N$-phenylbenzene-1-sulfonamide (150 mg, 0.61 mmol), (4-bromobut-3-yn-1-yl)benzene, (1R,2R)-N,N'-dimethyl-1,2-cyclohexanediamine as ligand, and heptane/EtOAc (90:10) as chromatography eluent. Entitled product 1k was obtained in $90 \%$ yield (206 mg) as colorless oil.

${ }^{1} \mathrm{H}$ NMR (400 MHz, Chloroform-d) $\delta=2.43(\mathrm{~s}, 3 \mathrm{H}), 2.61(\mathrm{t}, J=7.3 \mathrm{~Hz}, 2 \mathrm{H}), 2.82(\mathrm{t}, J=7.3 \mathrm{~Hz}, 2 \mathrm{H}), 7.13-7.17(\mathrm{~m}$, $4 \mathrm{H}), 7.21-7.27(\mathrm{~m}, 8 \mathrm{H}), 7.45-7.48(\mathrm{~m}, 2 \mathrm{H})$.<smiles>[3H-]N([As])C#CCCc1ccccc1</smiles>

$N$-(tert-butyl)-4-methyl-N-(4-phenylbut-1-yn-1-yl)benzenesulfonamide (1I) ${ }^{5}$

\footnotetext{
${ }^{4}$ Takimoto, M.; Gholap, S. S.; Hou, Z. Chem. Eur. J., 2015, 21, 15218.

${ }^{5}$ Harkat, H.; Borghèse, S.; De Nigris, M.; Kiselev, S.; Bénéteau, V.; Pale, P. Adv. Synth. Catal. 2014, 356, 3842.

${ }^{6}$ Xu, W.; Wang, G.; Sun, N.; Liu, Y. Org. Lett. 2017, 19, 3307.
} 
Synthesized following the general procedure 2.1 using 4-methyl- $N$-phenylbenzene-1-sulfonamide (200 mg, 0.88 mmol), (4-bromobut-3-yn-1-yl)benzene, (1R,2R)- $N, N^{\prime}$-dimethyl-1,2-cyclohexanediamine as ligand, and heptane/EtOAc (90:10) as chromatography eluent. Entitled product 1 l was obtained in $74 \%$ yield (232 mg) as colorless oil.

${ }^{1} \mathrm{H}$ NMR (400 MHz, Chloroform-d) $\delta=1.34(\mathrm{~m}, 9 \mathrm{H}), 2.42(\mathrm{~s}, 3 \mathrm{H}), 2.61(\mathrm{t}, \mathrm{J}=7.3 \mathrm{~Hz}, 2 \mathrm{H}), 2.81(\mathrm{t}, \mathrm{J}=7.4 \mathrm{~Hz}, 2 \mathrm{H}), 7.18$ $-7.21(\mathrm{~m}, 3 \mathrm{H}), 7.25-7.30(\mathrm{~m}, 4 \mathrm{H}), 7.73-7.76(\mathrm{~m}, 2 \mathrm{H}) .{ }^{13} \mathrm{C} \mathrm{NMR}\left(101 \mathrm{MHz}, \mathrm{CDCl}_{3}\right) \delta=21.7,29.3,30.0,35.4,63.4$, $71.2,74.0,126.3,127.7,128.5,128.6,129.4,138.0,140.9,143.8$.

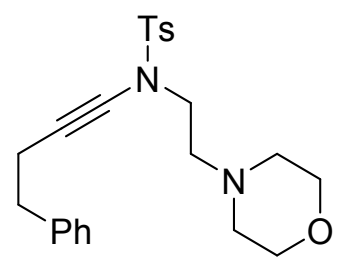

\section{4-methyl-N-(2-morpholinoethyl)-N-(4-phenylbut-1-yn-1-yl)benzenesulfonamide (1m)}

Synthesized following the general procedure 2.1 using 4-methyl- $N$-[2-(morpholin-4-yl)ethyl]benzene-1sulfonamide (142 mg, $0.50 \mathrm{mmol})$, (4-bromobut-3-yn-1-yl)benzene, $(1 R, 2 R)-N, N^{\prime}$-dimethyl-1,2cyclohexanediamine as ligand, and heptane/EtOAc (40:60) as chromatography eluent. Entitled product 1m was obtained in $87 \%$ yield (180 $\mathrm{mg}$ ) as colorless oil.

${ }^{1} \mathrm{H}$ NMR $(400 \mathrm{MHz}$, Chloroform-d) $\delta=2.38-2.40(\mathrm{~m}, 4 \mathrm{H}), 2.44(\mathrm{~s}, 3 \mathrm{H}), 2.49(\mathrm{t}, J=6.9 \mathrm{~Hz}, 2 \mathrm{H}), 2.58(\mathrm{t}, J=7.2 \mathrm{~Hz}$, $2 \mathrm{H}), 2.79(\mathrm{t}, J=7.3 \mathrm{~Hz}, 2 \mathrm{H}), 3.36(\mathrm{t}, J=6.9 \mathrm{~Hz}, 2 \mathrm{H}), 3.62-3.64(\mathrm{~m}, 4 \mathrm{H}), 7.16-7.23(\mathrm{~m}, 3 \mathrm{H}), 7.25-7.30(\mathrm{~m}, 4 \mathrm{H}), 7.71$ - $7.74(\mathrm{~m}, 2 \mathrm{H}) ;{ }^{13} \mathrm{C}$ NMR $\left(101 \mathrm{MHz}, \mathrm{CDCl}_{3}\right) \delta=20.6,21.8,35.3,48.7,53.7,56.1,67.0,69.6,74.0,126.4,127.8$, 128.5, 128.6, 129.7, 135.0, 140.7, 144.4.; HRMS (ESI): calculated for: $\mathrm{C}_{23} \mathrm{H}_{29} \mathrm{~N}_{2} \mathrm{O}_{3} \mathrm{~S}[\mathrm{M}+\mathrm{H}]^{+}: 413,1893$, found 413.1897.<smiles>COC(=O)CN([13CH3])C#CCCc1ccccc1</smiles>

methyl $\mathbf{N}$-(4-phenylbut-1-yn-1-yl)-N-tosylglycinate (1n)

Synthesized following the general procedure 2.1 using methyl 2-[(4-methylphenyl)sulfonamido]acetate (50 $\mathrm{mg}$, mmol), (4-bromobut-3-yn-1-yl)benzene, $(1 R, 2 R)-N, N^{\prime}$-dimethyl-1,2-cyclohexanediamine as ligand, and heptane/EtOAc (80:20) as chromatography eluent. Entitled product 1n was obtained in $50 \%$ yield (38 mg) as colorless oil.

${ }^{1} \mathrm{H}$ NMR (400 MHz, Chloroform-d) $\delta=2.43(\mathrm{~s}, 3 \mathrm{H}), 2.52(\mathrm{t}, \mathrm{J}=7.3 \mathrm{~Hz}, 2 \mathrm{H}), 2.76(\mathrm{t}, \mathrm{J}=7.4 \mathrm{~Hz}, 2 \mathrm{H}), 3.65(\mathrm{~s}, 3 \mathrm{H}), 4.14$ $(\mathrm{s}, 2 \mathrm{H}), 7.15-7.21(\mathrm{~m}, 3 \mathrm{H}), 7.24-7.29(\mathrm{~m}, 4 \mathrm{H}), 7.69-7.71(\mathrm{~m}, 2 \mathrm{H}) . ;{ }^{13} \mathrm{C}$ NMR $\left(101 \mathrm{MHz}, \mathrm{CDCl}_{3}\right) \delta=20.7,21.8$, 35.2, 52.4, 52.5, 69.5, 73.8, 76.8, 126.3, 128.1, 128.4, 128.6, 129.6, 134.7, 140.6, 144.7, 167.8; HRMS (ESI): calculated for $\mathrm{C}_{20} \mathrm{H}_{22} \mathrm{NO}_{4} \mathrm{~S}[\mathrm{M}+\mathrm{H}]^{+}: 372,1264$, found 372.1260 . 
3-(phenylethynyl)oxazolidin-2-one (10)<smiles>O=C1OCCN1C#Cc1ccccc1</smiles>

Synthesized following the general procedure 2.1 using oxazolidinone $(1.70 \mathrm{~g}, 19.52 \mathrm{mmol})$, (bromoethynyl)benzene, 1,10-phenanthroline as ligand, and heptane/EtOAc (80:20) as chromatography eluent. Entitled product 10 was obtained in $70 \%$ yield $(2.79 \mathrm{~g})$ as white solid.

${ }^{1} \mathrm{H}$ NMR $(400 \mathrm{MHz}$, Chloroform- $d) \delta=7.46-7.42(\mathrm{~m}, 2 \mathrm{H}), 7.32-7.29(\mathrm{~m}, 3 \mathrm{H})$, 4.51-4.47 (m, 2H), 4.03-3.99 (m, 2H).<smiles>O=C1OCCN1C#CC1CCCCC1</smiles>

3-(cyclohexylethynyl)oxazolidin-2-one (1p) ${ }^{8}$

Synthesized following the general procedure 2.1 using oxazolidinone $(1.16 \mathrm{~g}, 13.33 \mathrm{mmol})$, (bromoethynyl)cyclohexane, 1,10-phenanthroline as ligand and heptane/EtOAc (80:20) as chromatography eluent. Entitled product $1 \mathbf{p}$ was obtained in $73 \%$ yield $(1.88 \mathrm{~g})$ as colorless oil.

${ }^{1} \mathrm{H}$ NMR $(400 \mathrm{MHz}$, Chloroform- $d$ ) $\delta=\delta 4.35$ (dd, $J=8.8 \mathrm{~Hz}$ and $8.0 \mathrm{~Hz}, 2 \mathrm{H}), 3.80$ (dd, $J=8.8 \mathrm{~Hz}$ and $8.0 \mathrm{~Hz}, 2 \mathrm{H}$ ), 2.45-2.37 (m, 1H), 1.76-1.71 (m, 2H), 1.66-1.57 (m, 2H), 1.49-1.12 (m, 6H).

\section{3-(3,3-dimethylbut-1-yn-1-yl)oxazolidin-2-one (1q)}<smiles>CC(C)(C)C#CN1CCOC1=O</smiles>

Synthesized following an adapted version of Evano's synthesis of ynamides starting from dibromo-olefines. ${ }^{9}$

In a pressure tube, oxazolidinone (749 mg, $8.6 \mathrm{mmol}), 1,1$-dibromo-3,3-dimethylbut-1-ene (3.12 g, $12.9 \mathrm{mmol})$, $\mathrm{Cs}_{2} \mathrm{CO}_{3}(11.29 \mathrm{~g}, 34.4 \mathrm{mmol})$, and copper(l) iodide $(204 \mathrm{mg}, 1.075 \mathrm{mmol})$. The tube was then evacuated under high vacuum and backfilled with argon. Dry and degassed DMF (16 mL) and $N, N^{\prime}$ - dimethylethylenediamine (161 $\mu \mathrm{L}, 1.6 \mathrm{mmol}$ ) were added, and the tube was then sealed. The suspension was heated at $70^{\circ} \mathrm{C}$ for $48 \mathrm{~h}$. The resulting dark suspension was cooled down and the crude reaction mixture was diluted with water, extracted with diethyl ether and the combined organic layers were washed with brine, dried over $\mathrm{MgSO}_{4}$, filtered and concentrated. The crude residue was purified by flash chromatography over silica gel using heptane/EtOAc $(80: 20)$ as eluent. Entitled product $1 \mathrm{q}$ was obtained in $33 \%$ yield $(560 \mathrm{mg})$ as a white solid.

${ }^{1} \mathrm{H}$ NMR $(400 \mathrm{MHz}$, Chloroform-d) $\delta=4.37(\mathrm{dd}, J=8.8 \mathrm{~Hz}$ and $7.2 \mathrm{~Hz}, 2 \mathrm{H}), 3.83(\mathrm{dd}, J=8.8$ and $7.2 \mathrm{~Hz}, 2 \mathrm{H}), 1.22$ $(s, 9 H)$.

\footnotetext{
${ }^{7}$ Hamada, T.; Ye, X.; Stahl, S. S. J. Am. Chem. Soc. 2008, 130, 833.

8 Jouvin, K., ; Couty, F. ; Evano, E. Org. Lett. 2010, 121, 43272.

9 Jouvin, K. ; Coste, A. ; Bayle, A. ; Legrand, F. ; Karthikeyan, G.; Tadiparthi, K.; Evano, G. Organomet. 2012, 31, 7933.
} 


\subsection{General procedures for the hydroformylation reaction of ynamides}

BiPhePhos: In a dry Schlenk glassware under argon were introduced $\mathrm{Rh}(\mathrm{CO})_{2}(\mathrm{acac})(2 \mathrm{~mol} \%)$ and anhydrous degassed Toluene $(1 \mathrm{~mL})$. Biphephos $(8 \mathrm{~mol} \%)$ was added and $\mathrm{CO}$ evolution was observed. Subsequent addition of ynamide was performed. The mixture was transferred via a syringe in a dry stainless autoclave under argon. The glassware was rinsed with anhydrous degassed toluene $(3 \times 3 \mathrm{~mL})$ to reach a final concentration of $0.03 \mathrm{M}$. The autoclave was purged with $\mathrm{CO}$ before setting the pressure at 10 bars: $1 / 5: \mathrm{H}_{2} / \mathrm{CO}$. The autoclave was then heated at $70{ }^{\circ} \mathrm{C}$ for $16 \mathrm{~h}$. After cooling and degassing, the reaction was concentrated under vacuum. The crude product was purified by flash column chromatography on silica gel.

Xantphos: In a dry Schlenk glassware under argon were introduced $\mathrm{Rh}(\mathrm{CO})_{2}(\mathrm{acac})(2 \mathrm{~mol} \%)$ and anhydrous degassed Toluene $(1 \mathrm{~mL}$ ). Xantphos (8 mol\%) was added and CO evolution was observed. Subsequent addition of ynamide was performed. The mixture was transferred via a syringe in a dry stainless autoclave under argon. The glassware was rinsed with anhydrous degassed toluene $(3 \times 3 \mathrm{~mL})$ to reach a final concentration of $0.03 \mathrm{M}$. The autoclave was purged with $\mathrm{H}_{2} / \mathrm{CO}(1: 1)$ before setting the pressure at 5 bar. The autoclave was then heated at $70{ }^{\circ} \mathrm{C}$ for $16 \mathrm{~h}$. After cooling and degassing, the reaction was concentrated under vacuum. The crude product was purified by flash column chromatography on silica gel.

All the aldehyde overall yields are reported in the manuscript.<smiles>CCN([Te-])/C(C=O)=C/CCc1ccccc1</smiles>

(Z)-N-ethyl-4-methyl-N-(1-oxo-5-phenylpent-2-en-2-yl)benzenesulfonamide (2a)

Synthesized following the general procedure 2.2, using $N$-ethyl-4-methyl- $N$-(4-phenylbut-1-yn-1yl)benzene-1-sulfonamide $1 \mathrm{a}\left(100 \mathrm{mg}, 0.31 \mathrm{mmol}\right.$ ), BiPhePhos as ligand, and pentane $/ \mathrm{CH}_{2} \mathrm{Cl}_{2} / \mathrm{Et}_{2} \mathrm{O}(50: 47: 3)$ as chromatography eluent. Entitled product 2 a (43 mg, 53\%) was obtained as colorless oil.

Scale-up : In a dry Schlenk glassware under argon were introduced $6.2 \mathrm{mg}$ of $\mathrm{Rh}(\mathrm{CO})_{2}(\mathrm{acac})(2 \mathrm{~mol} \%, 0.024 \mathrm{mmol})$ and anhydrous degassed toluene $(10 \mathrm{~mL}) .77 \mathrm{mg}$ of BiPhePhos $(8 \mathrm{~mol} \%, 0.098 \mathrm{mmol})$ were added and CO evolution was observed. Subsequent addition of ynamide 1 a ( $400 \mathrm{mg}, 1.22 \mathrm{mmol}$ ) was performed. The mixture was transferred via a syringe in a dry stainless autoclave under argon. The glassware was rinsed with anhydrous degassed toluene $(3 \times 10 \mathrm{~mL})$ to reach a final concentration of $0.03 \mathrm{M}$. The autoclave was purged with CO before setting the pressure at 10 bars: $1 / 5: \mathrm{H}_{2} / \mathrm{CO}$. The autoclave was heated at $70{ }^{\circ} \mathrm{C}$ (internal temperature) overnight. After cooling and degassing, the reaction was concentrated under vacuum. The crude product was purified by flash column chromatography on silica gel (eluent pentane/ $\mathrm{CH}_{2} \mathrm{Cl}_{2} / \mathrm{Et}_{2} \mathrm{O}: 50: 47: 3$ ) to afford $2 \mathrm{a}$ (214 $\mathrm{mg}$, 49\%) as colorless oil.

${ }^{1} \mathbf{H}$ NMR $\left(400 \mathrm{MHz}, \mathrm{CDCl}_{3}\right): \delta=0.95(\mathrm{t}, J=7.2 \mathrm{~Hz}, 3 \mathrm{H}), 2.43(\mathrm{~s}, 3 \mathrm{H}), 2.94(\mathrm{bs}, 4 \mathrm{H}), 3.24(\mathrm{bs}, 1 \mathrm{H}), 3.56$ (bs, $\left.1 \mathrm{H}\right), 6.96$ $(\mathrm{m}, 1 \mathrm{H}), 7.22-7.36(\mathrm{~m}, 7 \mathrm{H}), 7.70(\mathrm{~m}, 2 \mathrm{H}), 9.25(\mathrm{~s}, 1 \mathrm{H}) ;{ }^{13} \mathrm{C} \mathrm{NMR}\left(101 \mathrm{MHz}, \mathrm{CDCl}_{3}\right): \delta=14.1,21.7,31.5,34.1,43.4$, 126.5, 127.8, 128.6, 128.7, 129.5, 136.8, 138.6, 140.4, 143.6, 159.8, 189.6; HRMS (ESI): calculated for $\mathrm{C}_{20} \mathrm{H}_{24} \mathrm{NO}_{3} \mathrm{~S}$ $[\mathrm{M}+\mathrm{H}]^{+}:$358.1471, found 358.1471 .<smiles>CCN([Te])/C=C/C(=O)[SiH3]</smiles>

(E)-N-ethyl- $N$-(2-formyl-4-phenylbut-1-en-1-yl)-4-methylbenzenesulfonamide (3a)

Synthesized following the general procedure 2.2, using $N$-ethyl-4-methyl- $N$-(4-phenylbut-1-yn-1yl)benzene-1-sulfonamide $1 \mathrm{a}(100 \mathrm{mg}, 0.31 \mathrm{mmol})$, Xantphos as ligand, and pentane $/ \mathrm{CH}_{2} \mathrm{Cl}_{2} / \mathrm{Et}_{2} \mathrm{O}(50: 47: 3)$ as chromatography eluent. Entitled product 3 a (75 mg, 69\%) was obtained as colorless oil. 
${ }^{1} \mathrm{H}$ NMR $\left(400 \mathrm{MHz}, \mathrm{CDCl}_{3}\right): \delta=1.11(\mathrm{t}, J=7.2 \mathrm{~Hz}, 3 \mathrm{H}), 2.47(\mathrm{~s}, 3 \mathrm{H}), 2.59(\mathrm{~m}, 4 \mathrm{H}), 3.48(\mathrm{q}, \mathrm{J}=7.2 \mathrm{~Hz}, 2 \mathrm{H}), 7.05-7.19$ $(\mathrm{m}, 5 \mathrm{H}), 7.37(\mathrm{~m}, 2 \mathrm{H}), 7.46(\mathrm{~s}, 1 \mathrm{H}), 7.69(\mathrm{~m}, 2 \mathrm{H}), 9.28(\mathrm{~s}, 1 \mathrm{H}) ;{ }^{13} \mathrm{C} \mathrm{NMR}\left(101 \mathrm{MHz}, \mathrm{CDCl}_{3}\right): \delta=14.7,21.8,25.7,35.6$, 41.8, 123.0, 126.3, 127.4, 128.4, 128.5, 130.4, 135.5, 141.1, 145.2, 146.8, 193.3; HRMS (ESI): calculated for $\mathrm{C}_{20} \mathrm{H}_{24} \mathrm{NO}_{3} \mathrm{~S}[\mathrm{M}+\mathrm{H}]^{+}: 358.1471$, found 358.1476 .<smiles>CCN([12F])/C(C=O)=C\CCO[R5](F)(F)F</smiles>

(Z)-N-(5-((tert-butyldimethylsilyl)oxy)-1-oxopent-2-en-2-yl)- $N$-ethyl-4-methylbenzenesulfonamide (2b)

Synthesized following the general procedure 2.2, using $\mathrm{N}$-\{4-[(tert-butyldimethy|silyl)oxy]but-1-yn-1-y|\}-N-ethyl-4-methylbenzene-1-sulfonamide $\mathbf{1 b}$ (100 $\mathrm{mg}, 0.26$ mmol), BiPhePhos as ligand, and $\mathrm{CH}_{2} \mathrm{Cl}_{2} / \mathrm{EtOAc}$ (99:1) as chromatography eluent. Entitled product $\mathbf{2 b}$ (50 $\mathrm{mg}$, 46\%) was obtained as colorless oil.

${ }^{1} \mathrm{H}$ NMR $(400 \mathrm{MHz}$, Chloroform-d) $\delta=0.07(\mathrm{~s}, 6 \mathrm{H}), 0.90(\mathrm{~s}, 9 \mathrm{H}), 1.04(\mathrm{t}, J=7.2 \mathrm{~Hz}, 3 \mathrm{H}), 2.41(\mathrm{~s}, 3 \mathrm{H}), 2.81(\mathrm{~d}, J=6.5$ $\mathrm{Hz}, 2 \mathrm{H}$ ), 3.26 (broad s, $1 \mathrm{H}$ ), 3.58 (broad s, $1 \mathrm{H}$ ), 3.83 (t, J=5.9 Hz, 2H), 7.13 (t, J=7.2 Hz, 1H), $7.25-7.28(\mathrm{~m}, 2 \mathrm{H}$ ), $7.66-7.69(\mathrm{~m}, 2 \mathrm{H}), 9.28(\mathrm{~s}, 1 \mathrm{H}) . ;{ }^{13} \mathrm{C}$ NMR $(101 \mathrm{MHz}$, Chloroform-d) $\delta=-5.2,14.3,18.4,21.7,26.0,33.4,43.5$, 61.1, 127.8, 129.5, 136.9, 139.1, 143.6, 158.9, 189.7; HRMS (ESI): calculated for $\mathrm{C}_{14} \mathrm{H}_{20} \mathrm{NO}_{4} \mathrm{~S}[\mathrm{M}-\mathrm{TBS}+2 \mathrm{H}]^{+}$: 298.1108 , found 298.1107.

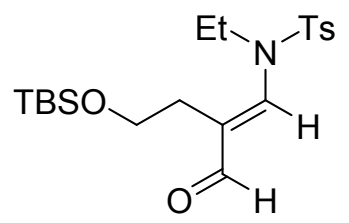

(E)-N-(4-((tert-butyldimethy|silyl)oxy)-2-formylbut-1-en-1-yl)-N-ethyl-4-methylbenzenesulfonamide (3b) Synthesized following the general procedure 2.2, using $N$-ethyl-4-methyl- $N$-(4-phenylbut-1-yn-1yl)benzene-1-sulfonamide $\mathbf{1 b}$ (100 mg, 0.26), Xantphos as ligand, and $\mathrm{CH}_{2} \mathrm{Cl}_{2} / \mathrm{EtOAc}$ (99:1) as chromatography eluent. Entitled product $\mathbf{3 b}(57 \mathrm{mg}, 54 \%)$ was obtained as colorless oil.

${ }^{1} \mathrm{H}$ NMR $(500 \mathrm{MHz}$, Chloroform-d) $\delta=-0.15(\mathrm{~s}, 6 \mathrm{H}), 0.72(\mathrm{~s}, 9 \mathrm{H}), 1.17(\mathrm{t}, \mathrm{J}=7.1 \mathrm{~Hz}, 3 \mathrm{H}), 2.45(\mathrm{~s}, 3 \mathrm{H}), 2.54(\mathrm{t}, J=5.9$ $\mathrm{Hz}, 2 \mathrm{H}), 3.59(\mathrm{t}, \mathrm{J}=5.9 \mathrm{~Hz}, 2 \mathrm{H}), 3.93(\mathrm{q}, J=7.1 \mathrm{~Hz}, 2 \mathrm{H}), 7.34-7.36(\mathrm{~m}, 2 \mathrm{H}), 7.60(\mathrm{~s}, 1 \mathrm{H}), 7.73-7.76(\mathrm{~m}, 2 \mathrm{H}), 9.25(\mathrm{~s}$, 1H); ${ }^{13}$ C NMR (126 MHz, Chloroform-d) $\delta=-5.5,14.8,18.3,21.7,26.0,26.7,41.7,61.9,119.3,127.5,130.3,135.5$, 145.1, 148.4, 193.6; HRMS (ESI): calculated for $\mathrm{C}_{20} \mathrm{H}_{34} \mathrm{NO}_{4} \mathrm{SSi}[\mathrm{M}+\mathrm{H}]^{+}:$: 412.1972, found 412.1971.<smiles>CCN([13CH3])/C(=C/C=O)c1ccccc1</smiles>

(Z)-N-ethyl-4-methyl-N-(3-oxo-1-phenylprop-1-en-2-yl)benzenesulfonamide (2c)

Synthesized following the general procedure 2.2, using $\mathrm{N}$-ethyl-4-methyl- $\mathrm{N}$-(2-phenylethynyl)benzene-1-sulfonamide $1 \mathrm{c}(100 \mathrm{mg}, 0.33 \mathrm{mmol})$, BiPhePhos as ligand, and $\mathrm{CH}_{2} \mathrm{Cl}_{2} /$ EtOAc $(97.5: 2.5)$ as chromatography eluent. Entitled product $2 \mathrm{c}(43 \mathrm{mg}, 39 \%)$ was obtained as colorless oil.

${ }^{1} \mathrm{H}_{\mathrm{NMR}}{ }^{1} \mathrm{H}$ NMR $(400 \mathrm{MHz}$, Chloroform- $d) \delta=1.01(\mathrm{t}, J=7.3 \mathrm{~Hz}, 3 \mathrm{H}), 2.43(\mathrm{~s}, 3 \mathrm{H}), 3.54(\mathrm{~s}, 2 \mathrm{H}), 7.28-7.31(\mathrm{~m}, 2 \mathrm{H})$, $7.41-7.48(\mathrm{~m}, 4 \mathrm{H}), 7.73-7.76(\mathrm{~m}, 2 \mathrm{H}), 7.94-7.96(\mathrm{~m}, 2 \mathrm{H}), 9.43(\mathrm{~s}, 1 \mathrm{H}) ;{ }^{13} \mathrm{C}$ NMR $\left(101 \mathrm{MHz}, \mathrm{CDCl}_{3}\right) \delta=13.8$, 21.7, 43.7, 128.2, 129.0, 129.5, 131.5, 132.1, 132.3, 135.5, 136.8, 143.8, 152.0, 190.8.; HRMS (ESI): calculated for $\mathrm{C}_{18} \mathrm{H}_{20} \mathrm{NO}_{3} \mathrm{~S}[\mathrm{M}+\mathrm{H}]^{+}: 330.1158$, found 330.1154 . 
<smiles>CCN(C)/C=C/C=O</smiles>

(E)-N-ethyl-4-methyl-N-(3-oxo-2-phenylprop-1-en-1-yl)benzenesulfonamide (3c)

Synthesized following the general procedure $N$-ethyl-4-methyl- $N$-(2-phenylethynyl)benzene-1-sulfonamide $1 \mathrm{c}(100 \mathrm{mg}, 0.33 \mathrm{mmol})$, Xantphos as ligand, and $\mathrm{CH}_{2} \mathrm{Cl}_{2}$ /EtOAc (97.5:2.5) as chromatography eluent. Entitled product $3 \mathrm{c}(87 \mathrm{mg}, 79 \%)$ was obtained as amorphous white solid.

${ }^{1} \mathbf{H}$ NMR (400 MHz, Chloroform- $d$ ) $\delta=0.64(\mathrm{t}, J=7.0 \mathrm{~Hz}, 3 \mathrm{H}), 2.47(\mathrm{~s}, 3 \mathrm{H}), 3.25$ (q, J=7.0 Hz, 2H), $7.05-7.07$ (m, $2 \mathrm{H}), 7.30-7.33(\mathrm{~m}, 3 \mathrm{H}), 7.39(\mathrm{~d}, \mathrm{~J}=8.0 \mathrm{~Hz}, 2 \mathrm{H}), 7.75-7.78(\mathrm{~m}, 3 \mathrm{H}), 9.48(\mathrm{~s}, 1 \mathrm{H}) ;{ }^{13} \mathrm{C} \mathrm{NMR}\left(101 \mathrm{MHz}, \mathrm{CDCl}_{3}\right) \delta=$ 13.6, 21.8, 41.9, 124.4, 127.4, 128.3, 128.4, 130.3, 130.4, 131.9, 135.5, 145.3, 146.1, 191.8.; HRMS (ESI): calculated for $\mathrm{C}_{18} \mathrm{H}_{20} \mathrm{NO}_{3} \mathrm{~S}[\mathrm{M}+\mathrm{H}]^{+}: 330.1158$, found 330.1157 .<smiles>CCN([13CH3])/C=C/C=O</smiles>

(E)-N-ethyl-4-methyl-N-(3-oxoprop-1-en-1-yl)benzenesulfonamide (3e)

Synthesized following the general procedure 2.2, using $\mathrm{N}$-ethyl- $\mathrm{N}$-ethynyl-4-methylbenzene-1-sulfonamide $\mathbf{1 e}$ (72 $\mathrm{mg}, 0.32 \mathrm{mmol})$, Xantphos as ligand, and heptane/EtOAc (75:25) as chromatography eluent. Entitled product 3e (44 $\mathrm{mg}, 54 \%)$ was obtained as amorphous white solid.

${ }^{1} \mathrm{H}$ NMR (400 MHz, Chloroform-d) $\delta=1.14(\mathrm{t}, \mathrm{J}=7.1 \mathrm{~Hz}, 3 \mathrm{H}), 2.46$ (s, 3H), 3.51 (d, J=7.1 Hz, 2H), 5.48 (dd, J=14.0, $7.8 \mathrm{~Hz}, 1 \mathrm{H}), 7.36-7.38(\mathrm{~m}, 2 \mathrm{H}), 7.71-7.73(\mathrm{~m}, 2 \mathrm{H}), 7.89(\mathrm{~d}, J=14.1,1 \mathrm{H}), 9.41(\mathrm{~d}, J=7.8 \mathrm{~Hz}, 1 \mathrm{H}) ;{ }^{13} \mathrm{C}$ NMR $(101$ $\mathrm{MHz}$, Chloroform-d) $\delta=12.2,21.8,41.5,110.2,127.4,130.5,135.1,145.5,149.2,191.2 ;$ HRMS (ESI): calculated for $\mathrm{C}_{12} \mathrm{H}_{16} \mathrm{NO}_{3} \mathrm{~S}[\mathrm{M}+\mathrm{H}]^{+}: 254.0845$, found 254.0843 .<smiles>CCN(C(=O)OCc1ccccc1)/C(C=O)=C\CCc1ccccc1</smiles>

tert-butyl (Z)-ethyl(1-oxo-5-phenylpent-2-en-2-yl)carbamate (2f)

Synthesized following the general procedure 2.2, using tert-butyl $N$-ethyl- $N$-(4-phenylbut-1-yn-1-yl)carbamate 1 if ( $35 \mathrm{mg}, 0.13 \mathrm{mmol}$ ), BiPhePhos as ligand, and $\mathrm{CH}_{2} \mathrm{Cl}_{2} / \mathrm{EtOAc}$ (99:1 to 90:10) as chromatography eluent. Entitled product $\mathbf{2 f}(16 \mathrm{mg}, 41 \%)$ was obtained as amorphous white solid.

${ }^{1} \mathrm{H}$ NMR $(400 \mathrm{MHz}$, Chloroform-d) $\delta=0.99(\mathrm{t}, J=7.2 \mathrm{~Hz}, 3 \mathrm{H}), 1.36-1.49(\mathrm{~m}, 9 \mathrm{H}), 2.57-2.69(\mathrm{~m}, 2 \mathrm{H}), 2.83(\mathrm{t}, J=7.2$ $\mathrm{Hz}, 2 \mathrm{H}), 3.24-3.43(\mathrm{~m}, 2 \mathrm{H}), 5.30(\mathrm{~s}, 1 \mathrm{H}), 6.51-6.66(\mathrm{dt}, J=45.1,7.2 \mathrm{~Hz}, 1 \mathrm{H}), 7.19-7.23(\mathrm{~m}, 3 \mathrm{H}), 7.30-7.34(\mathrm{~m}$, 2H), $9.32-9.34(\mathrm{~m}, 1 \mathrm{H}) ;{ }^{13} \mathrm{C}$ NMR (101 MHz, Chloroform-d) $\delta=13.5,14.2,28.3,28.5,30.1,30.6,34.0,34.2,42.8$, 43.6, 80.4, 126.5, 126.6, 128.5, 128.5, 128.8, 140.3, 150.4, 152.2, 154.2, 190.0, 190.2; HRMS (ESI): calculated for $\mathrm{C}_{18} \mathrm{H}_{25} \mathrm{NNaO}_{3}[\mathrm{M}+\mathrm{Na}]^{+}: 326.1732$, found 326.1716 .<smiles>CCN(C=CC(=O)N(CC)C(=O)OCc1ccccc1)C(=O)c1ccccc1</smiles>

tert-butyl (E)-ethyl(2-formyl-4-phenylbut-1-en-1-yl)carbamate (3f)

Synthesized following the general procedure 2.2, using tert-butyl $N$-ethyl- $N$-(4-phenylbut-1-yn-1-yl)carbamate $\mathbf{1 f}$ (52 mg, $0.19 \mathrm{mmol}$ ), Xantphos as ligand, and $\mathrm{CH}_{2} \mathrm{Cl}_{2} / \mathrm{EtOAc}$ (99:1 to $90: 10$ ) as chromatography eluent. Entitled product $\mathbf{3 f}$ (35 mg, 61\%) was obtained as colorless oil. 
${ }^{1} \mathrm{H}$ NMR (400 MHz, Chloroform-d) $\delta=1.18(\mathrm{t}, J=7.0 \mathrm{~Hz}, 3 \mathrm{H}), 1.54(\mathrm{~s}, 9 \mathrm{H}), 2.61-2.72(\mathrm{~m}, 4 \mathrm{H}), 3.69(\mathrm{q}, J=7.1 \mathrm{~Hz}$, $2 \mathrm{H}), 7.17-7.21(\mathrm{~m}, 3 \mathrm{H}), 7.25-7.30(\mathrm{~m}, 2 \mathrm{H}), 7.56(\mathrm{~s}, 1 \mathrm{H}), 9.24(\mathrm{~s}, 1 \mathrm{H}) ;{ }^{13} \mathrm{C}$ NMR $(101 \mathrm{MHz}$, Chloroform-d) $\delta=$ 14.5, 26.0, 28.2, 35.7, 41.0, 83.5, 122.3, 126.3, 128.5, 128.6, 141.6, 148.8, 152.7, 194.2; HRMS (ESI): calculated for $\mathrm{C}_{18} \mathrm{H}_{25} \mathrm{NNaO}_{3}[\mathrm{M}+\mathrm{Na}]^{+}$: 326.1732, found 326.1719.<smiles>CCN(C(=O)OC)/C(C=O)=C\CCc1ccccc1</smiles>

methyl (Z)-ethyl(1-oxo-5-phenylpent-2-en-2-yl)carbamate (2g)

Synthesized following the general procedure 2.2, using methyl $N$-ethyl- $N$-(4-phenylbut-1-yn-1-yl)carbamate $\mathbf{1 g}$ (83 mg, $0.36 \mathrm{mmol}$ ), BiPhePhos as ligand, and $\mathrm{CH}_{2} \mathrm{Cl}_{2} / \mathrm{EtOAc}$ (99:1 to 90:10) as chromatography eluent. Entitled product $2 \mathrm{~g}$ (43 $\mathrm{mg}, 46 \%$ ) was obtained as thick yellow oil.

${ }^{1} \mathbf{H}$ NMR (400 MHz, Chloroform-d) $\delta=0.98-1.03(\mathrm{~m}, 3 \mathrm{H}), 2.61-2.67(\mathrm{~m}, 2 \mathrm{H}), 2.81-2.87(\mathrm{~m}, 2 \mathrm{H}), 3.33-3.41$ $(\mathrm{m}, 2 \mathrm{H}), 3.60-3.75(\mathrm{~m}, 3 \mathrm{H}), 6.58-6.71(\mathrm{~m}, 1 \mathrm{H}), 7.17-7.25(\mathrm{~m}, 3 \mathrm{H}), 7.29-7.33(\mathrm{~m}, 2 \mathrm{H}), 9.33-9.35(\mathrm{~m}, 1 \mathrm{H}) ;{ }^{13} \mathrm{C}$ NMR $(101 \mathrm{MHz}$, Chloroform-d) $\delta=13.4,14.1,30.3,30.5,34.1,43.4,43.6,53.0,53.1,126.7,128.5,128.5,128.8$, 151.6, 152.8, 189.8, 189.9; HRMS (ESI): calculated for $\mathrm{C}_{15} \mathrm{H}_{20} \mathrm{NO}_{3}[\mathrm{M}+\mathrm{H}]^{+}:$:262.1438, found 262.1429.<smiles>CCN(/C=C\C(=O)CCc1ccccc1)C(=O)OC</smiles>

methyl (E)-ethyl(2-formyl-4-phenylbut-1-en-1-yl)carbamate (3g)

Synthesized following the general procedure 2.2, using methyl $N$-ethyl- $N$-(4-phenylbut-1-yn-1-yl)carbamate $\mathbf{1 g}$ (100 mg, $0.43 \mathrm{mmol}$ ), Xantphos as ligand, and $\mathrm{CH}_{2} \mathrm{Cl}_{2} / \mathrm{EtOAc}$ (99:1 to 90:10) as chromatography eluent. Entitled product $3 \mathrm{~g}$ ( $86 \mathrm{mg}, 76 \%$ ) was obtained as amorphous white solid.

${ }^{1} \mathrm{H}$ NMR (400 MHz, Chloroform-d) $\delta=1.20(\mathrm{t}, \mathrm{J}=7.1 \mathrm{~Hz}, 3 \mathrm{H}), 2.63-2.73(\mathrm{~m}, 4 \mathrm{H}), 3.73(\mathrm{q}, \mathrm{J}=7.1 \mathrm{~Hz}, 2 \mathrm{H}), 3.88(\mathrm{~s}$, $3 \mathrm{H}), 7.18-7.22(\mathrm{~m}, 3 \mathrm{H}), 7.27-7.31(\mathrm{~m}, 2 \mathrm{H}), 7.54(\mathrm{~s}, 1 \mathrm{H}), 9.28(\mathrm{~s}, 1 \mathrm{H}) ;{ }^{13} \mathrm{C}$ NMR $(101 \mathrm{MHz}$, Chloroform-d) $\delta=$ 14.4, 26.0, 35.6, 41.3, 54.4, 123.6, 126.4, 128.5, 141.4, 148.3, 154.6, 194.0; HRMS (ESI): calculated for $\mathrm{C}_{15} \mathrm{H}_{20} \mathrm{NO}_{3}$ $[\mathrm{M}+\mathrm{H}]^{+}: 262.1438$, found 262.1433 .<smiles>O=C/C(=C/CCc1ccccc1)N1CCOC1=O</smiles>

(Z)-2-(2-oxooxazolidin-3-yl)-5-phenylpent-2-enal (2h)

Synthesized following the general procedure 2.2, using 3-(4-phenylbut-1-yn-1-yl)-1,3-oxazolidin-2-one $\mathbf{1 h}$ (100 $\mathrm{mg}, 0.46 \mathrm{mmol})$, BiPhePhos as ligand, and $\mathrm{CH}_{2} \mathrm{Cl}_{2} /$ EtOAc (92.5:7.5) as chromatography eluent. Entitled product 2h (54 mg, 47\%) was obtained as colorless oil.

${ }^{1} \mathrm{H}$ NMR (400 MHz, Chloroform-d) $\delta=2.74(\mathrm{qd}, J=7.3,0.9 \mathrm{~Hz}, 2 \mathrm{H}), 2.89(\mathrm{t}, J=7.3 \mathrm{~Hz}, 2 \mathrm{H}), 3.57-3.61(\mathrm{~m}, 2 \mathrm{H}), 4.38$ $-4.42(\mathrm{~m}, 2 \mathrm{H}), 6.74(\mathrm{t}, J=7.4 \mathrm{~Hz}, 1 \mathrm{H}), 7.19-7.24(\mathrm{~m}, 3 \mathrm{H}), 7.28-7.33(\mathrm{~m}, 2 \mathrm{H}), 9.34(\mathrm{~s}, 1 \mathrm{H}) ;{ }^{13} \mathrm{C} \mathrm{NMR}(101 \mathrm{MHz}$, Chloroform- $d$ ) $\delta=30.8$, 34.0, 45.2, 63.1, 126.7, 128.6, 128.8, 140.4, 153.7, 189.1; HRMS (ESI): calculated for $\mathrm{C}_{14} \mathrm{H}_{16} \mathrm{NO}_{3}[\mathrm{M}+\mathrm{H}]^{+}:$246.1125, found 246.1116. 
<smiles>O=C/C=C\N1CCOC1=O</smiles>

(E)-2-((2-oxooxazolidin-3-yl)methylene)-4-phenylbutanal (3h)

Synthesized following the general procedure 2.2, using 3-(4-phenylbut-1-yn-1-yl)-1,3-oxazolidin-2-one $1 \mathrm{~h}$ (100 $\mathrm{mg}, 0.46 \mathrm{mmol}$ ), Xantphos as ligand, and $\mathrm{CH}_{2} \mathrm{Cl}_{2} /$ EtOAc (92.5:7.5) as chromatography eluent. Entitled product $3 \mathrm{~h}$ (73 $\mathrm{mg}, 64 \%$ ) was obtained as amorphous white solid.

${ }^{1} \mathrm{H}$ NMR $(400 \mathrm{MHz}$, Chloroform-d) $\delta=2.62-2.66(\mathrm{~m}, 2 \mathrm{H}), 2.71-2.75(\mathrm{~m}, 2 \mathrm{H}), 3.61-3.65(\mathrm{~m}, 2 \mathrm{H}), 4.25-4.29$ $(\mathrm{m}, 2 \mathrm{H}), 7.12-7.15(\mathrm{~m}, 2 \mathrm{H}), 7.19-7.24(\mathrm{~m}, 1 \mathrm{H}), 7.26-7.30(\mathrm{~m}, 2 \mathrm{H}), 9.23(\mathrm{~s}, 1 \mathrm{H}) ;{ }^{13} \mathrm{C} \mathrm{NMR}(101 \mathrm{MHz}$, Chloroformd) $\delta=25.2,35.5,43.6,62.7,123.0,126.5,128.6,128.8,141.1,143.9,155.4,193.2 ;$ HRMS (ESI): calculated for $\mathrm{C}_{14} \mathrm{H}_{16} \mathrm{NO}_{3}[\mathrm{M}+\mathrm{H}]^{+}: 246.1125$, found 246.1117 .<smiles>O=C/C(=C/CCc1ccccc1)N1C(=O)OC[C@H]1Br</smiles>

(S,Z)-2-(4-benzyl-2-oxooxazolidin-3-yl)-5-phenylpent-2-enal (2i)

Synthesized following the general procedure 2.2, using (4S)-4-benzyl-3-(4-phenylbut-1-yn-1-yl)-1,3-oxazolidin-2-one $1 \mathbf{i}(100 \mathrm{mg}, 0.33 \mathrm{mmol})$, BiPhePhos as ligand, and heptane/EtOAc (80:20) as chromatography eluent. Entitled product $2 \mathbf{i}(47 \mathrm{mg}, 43 \%)$ was obtained as amorphous beige solid.

${ }^{1} \mathrm{H}$ NMR $(500 \mathrm{MHz}$, Chloroform-d) $\delta=2.45(\mathrm{dd}, J=13.5,9.5 \mathrm{~Hz}, 1 \mathrm{H}), 2.61-2.71(\mathrm{~m}, 2 \mathrm{H}), 2.80-2.88(\mathrm{~m}, 2 \mathrm{H}), 2.91$ $-2.98(\mathrm{~m}, 1 \mathrm{H}), 4.08(\mathrm{dd}, J=8.7,6.9 \mathrm{~Hz}, 1 \mathrm{H}), 4.36(\mathrm{t}, J=8.5 \mathrm{~Hz}, 1 \mathrm{H}), 4.67-4.73(\mathrm{~m}, 1 \mathrm{H}), 6.73-6.75(\mathrm{~m}, 1 \mathrm{H}), 7.02-$ $7.05(\mathrm{~m}, 2 \mathrm{H}), 7.19-7.33(\mathrm{~m}, 8 \mathrm{H}), 9.30(\mathrm{~s}, 1 \mathrm{H}) ;{ }^{13} \mathrm{C}$ NMR $(126 \mathrm{MHz}$, Chloroform-d) $\delta=31.2,34.0,39.7,56.5,68.4$, 126.7, 127.4, 128.6, 128.8, 128.9, 129.0, 13 5.3, 136.1, 140.3, 155.0, 155.8, 189.4; HRMS (ESI): calculated for $\mathrm{C}_{21} \mathrm{H}_{22} \mathrm{NO}_{3}[\mathrm{M}+\mathrm{H}]^{+}:$336.1594, found 336.1592; $[\propto]_{D}^{18}=-116.0$ (c 1.00, DMF).<smiles>O=C/C=C\N1C(=O)OC[C@H]1Br</smiles>

(S,E)-2-((4-benzyl-2-oxooxazolidin-3-yl)methylene)-4-phenylbutanal (3i)

Synthesized following the general procedure (4S)-4-benzyl-3-(4-phenylbut-1-yn-1-yl)-1,3-oxazolidin-2-one $1 \mathbf{i}(100 \mathrm{mg}, 0.33 \mathrm{mmol})$, Xantphos as ligand, and heptane/EtOAc (80:20) as chromatography eluent. Entitled product $3 \mathbf{i}(83 \mathrm{mg}, 76 \%)$ was obtained as amorphous off white solid.

${ }^{1} \mathrm{H}$ NMR $(400 \mathrm{MHz}$, Chloroform- $d) \delta=2.33-2.41(\mathrm{~m}, 1 \mathrm{H}), 2.57-2.66(\mathrm{~m}, 2 \mathrm{H}), 2.92-2.99(\mathrm{~m}, 2 \mathrm{H}), 3.16$ (ddd, $J=13.5,6.5,5.6 \mathrm{~Hz}, 1 \mathrm{H}), 3.77-3.81(\mathrm{~m}, 1 \mathrm{H}), 3.95-4.01(\mathrm{~m}, 1 \mathrm{H}), 4.08(\mathrm{dd}, J=8.8,1.7 \mathrm{~Hz}, 1 \mathrm{H}), 7.03-7.05(\mathrm{~m}, 2 \mathrm{H})$, $7.13-7.16(\mathrm{~m}, 2 \mathrm{H}), 7.18-7.22(\mathrm{~m}, 1 \mathrm{H}), 7.24-7.34(\mathrm{~m}, 6 \mathrm{H}), 7.40(\mathrm{~s}, 1 \mathrm{H}) ., 9.44(\mathrm{~d}, \mathrm{~J}=1.1 \mathrm{~Hz}, 1 \mathrm{H}) ;{ }^{13} \mathrm{C} \mathrm{NMR}(101$ $\mathrm{MHz}$, Chloroform-d) $\delta=25.9,35.0,37.6,54.9,66.4,123.1,126.7,127.9,128.8,129.0,129.2,129.3,134.1,141.0$, 143.1, 154.9, 193.3; HRMS (ESI): calculated for $\mathrm{C}_{21} \mathrm{H}_{22} \mathrm{NO}_{3}[\mathrm{M}+\mathrm{H}]^{+}: 336.1594$, found 336.1586; $[\propto]_{D}^{18}=-60.0(c$ $1.00, \mathrm{DMF})$. 
<smiles>CN(Br)/C(C=O)=C\CCc1ccccc1</smiles>

(Z)-N-benzyl-4-methyl-N-(1-oxo-5-phenylpent-2-en-2-yl)benzenesulfonamide (2j)

Synthesized following the general procedure 2.2, using $N$-benzyl-4-methyl- $N$-(4-phenylbut-1-yn-1$\mathrm{yl}$ )benzenesulfonamide $1 \mathrm{j}$ (41 mg, $0.11 \mathrm{mmol})$, BiPhePhos as ligand, and pentane/ $\mathrm{Et}_{2} \mathrm{O}(80: 20$ to $75 / 25)$ as chromatography eluent. Entitled product $\mathbf{2 j}$ ( $21 \mathrm{mg}, 47 \%)$ was obtained as amorphous off white solid.

${ }^{1} \mathrm{H}$ NMR $(400 \mathrm{MHz}$, Chloroform-d) $\delta=2.34-2.43(\mathrm{~m}, 5 \mathrm{H}), 2.58-2.62(\mathrm{~s}, 2 \mathrm{H}), 4.23$ (broad s, 1H), 4.87 (bs, 1H), $6.70(\mathrm{t}, J=7.3 \mathrm{~Hz}, 1 \mathrm{H}), 7.02-7.04(\mathrm{~m}, 2 \mathrm{H}), 7.17-7.30(\mathrm{~m}, 10 \mathrm{H}), 7.69-7.72(\mathrm{~m}, 2 \mathrm{H}), 9.08(\mathrm{~s}, 1 \mathrm{H}) ;{ }^{13} \mathrm{C}$ NMR $(101$ $\mathrm{MHz}$, Chloroform-d): $\delta=21.7,31.3,33.7,52.0,126.4,127.8,128.3,128.5,128.6,128.7,129.6,129.7,135.7$, 136.9, 137.9, 140.3, 143.8, 161.1, 189.6; HRMS (ESI): calculated for $\mathrm{C}_{25} \mathrm{H}_{25} \mathrm{NNaO}_{3} \mathrm{~S}[\mathrm{M}+\mathrm{Na}]^{+}: 442.1453$, found 442.1443 .<smiles>O=C/C=C\CCc1ccccc1</smiles>

(E)-N-benzyl- $N$-(2-formyl-4-phenylbut-1-en-1-yl)-4-methylbenzenesulfonamide (3j)

Synthesized following the general procedure 2.2, using $N$-benzyl-4-methyl- $N$-(4-phenylbut-1-yn-1$\mathrm{yl}$ )benzenesulfonamide $\mathbf{1 j}$ (100 mg, $0.26 \mathrm{mmol})$, Xantphos as ligand, and pentane/ $\mathrm{Et}_{2} \mathrm{O}(80: 20$ to $75 / 25)$ as chromatography eluent. Entitled product $\mathbf{3 j}$ ( $75 \mathrm{mg}, 70 \%)$ was obtained as colorless oil.

${ }^{1} \mathrm{H}$ NMR $(400 \mathrm{MHz}$, Chloroform- $d) \delta=2.33-2.37(\mathrm{~m}, 2 \mathrm{H}), 2.43-2.55(\mathrm{~m}, 5 \mathrm{H}), 4.57(\mathrm{~s}, 2 \mathrm{H}), 6.94-6.96(\mathrm{~m}, 2 \mathrm{H})$, $7.07-7.10(\mathrm{~m}, 2 \mathrm{H}), 7.13-7.18(\mathrm{~m}, 3 \mathrm{H}), 7.26-7.34(\mathrm{~m}, 3 \mathrm{H}), 7.38(\mathrm{~d}, \mathrm{~J}=8.1 \mathrm{~Hz}, 2 \mathrm{H}), 7.65(\mathrm{~s}, 1 \mathrm{H}), 7.68-7.71(\mathrm{~m}$, 2H), $9.34(\mathrm{~s}, 1 \mathrm{H}) ;{ }^{13} \mathrm{C}$ NMR ${ }^{13} \mathrm{C}$ NMR $(101 \mathrm{MHz}$, Chloroform-d) $\delta=21.8,25.4,35.3,50.0,123.9,125.9,126.2,127.5$, 127.8, 128.4, 128.6, 129.0, 130.3, 135.2, 135.5, 141.2, 145.3, 147.4, 193.4; HRMS (ESI): calculated for $\mathrm{C}_{25} \mathrm{H}_{26} \mathrm{NO}_{3} \mathrm{~S}$ $[\mathrm{M}+\mathrm{H}]^{+}:$420.1628, found 420.1624 .<smiles>O=C/C(=C/CCc1ccccc1)N([Te])c1ccccc1</smiles>

(Z)-4-methyl-N-(1-oxo-5-phenylpent-2-en-2-yl)- $N$-phenylbenzenesulfonamide (2k)

Synthesized following the general procedure 2.2, using 4-methyl- $N$-phenyl- $N$-(4-phenylbut-1-yn-1yl)benzenesulfonamide 1k (90 mg, $0.24 \mathrm{mmol}$ ), BiPhePhos as ligand, and heptane/EtOAc (90:10 to 80:20) as chromatography eluent. Entitled product $\mathbf{2 k}$ ( $44 \mathrm{mg}, 45 \%$ ) was obtained as amorphous yellowish solid.

${ }^{1} \mathrm{H}$ NMR $(500 \mathrm{MHz}$, Chloroform-d) $\delta=2.39(\mathrm{~s}, 3 \mathrm{H}), 2.78(\mathrm{t}, J=7.6 \mathrm{~Hz}, 2 \mathrm{H}), 2.94(\mathrm{q}, J=7.4 \mathrm{~Hz}, 2 \mathrm{H}), 6.89(\mathrm{t}, J=7.4 \mathrm{~Hz}$, $1 \mathrm{H}), 7.15-7.17(\mathrm{~m}, 2 \mathrm{H}), 7.19-7.25(\mathrm{~m}, 8 \mathrm{H}), 7.28-7.31(\mathrm{~m}, 2 \mathrm{H}), 7.65-7.66(\mathrm{~m}, 2 \mathrm{H}), 9.44(\mathrm{~s}, 1 \mathrm{H}) ;{ }^{13} \mathrm{C}$ NMR $(126$ $\mathrm{MHz}$, Chloroform-d) $\delta=21.7,30.9,33.8,126.6,127.0,127.5,128.5,128.6,128.8,129.3,129.3,136.5,139.8$, 140.2, 142.1, 143.9, 157.6, 190.2; HRMS (ESI): calculated for $\mathrm{C}_{24} \mathrm{H}_{23} \mathrm{NNaO}_{3} \mathrm{~S}[\mathrm{M}+\mathrm{Na}]^{+}$: 428.1296, found 428.1298.<smiles>C=C(/C=C\C=O)N([Te])c1ccccc1</smiles>

(E)-N-(2-formyl-4-phenylbut-1-en-1-yl)-4-methyl- $N$-phenylbenzenesulfonamide (3k)

Synthesized following the general procedure 2.2, using 4-methyl- $N$-phenyl- $N$-(4-phenylbut-1-yn-1yl)benzenesulfonamide $\mathbf{1 k}$ (90 mg, $0.24 \mathrm{mmol}$ ), Xantphos as ligand, and heptane/EtOAc (90:10 to 80:20) as chromatography eluent. Entitled product $\mathbf{3 k}$ (70 mg, 72\%) was obtained as amorphous white solid. 
${ }^{1} \mathrm{H}$ NMR $(500 \mathrm{MHz}$, Chloroform-d) $\delta=1.68-1.71(\mathrm{~m}, 2 \mathrm{H}), 2.19-2.22(\mathrm{~m}, 2 \mathrm{H}), 2.45(\mathrm{~s}, 3 \mathrm{H}), 6.74-6.76(\mathrm{~m}, 2 \mathrm{H})$, $6.99-7.01(\mathrm{~m}, 2 \mathrm{H}), 7.07-7.10(\mathrm{~m}, 1 \mathrm{H}), 7.12-7.15(\mathrm{~m}, 2 \mathrm{H}), 7.30(\mathrm{~d}, J=8.0 \mathrm{~Hz}, 2 \mathrm{H}), 7.34-7.34(\mathrm{~m}, 2 \mathrm{H}), 7.42-$ $7.46(\mathrm{~m}, 1 \mathrm{H}), 7.51-7.52(\mathrm{~m}, 2 \mathrm{H}), 7.74(\mathrm{~s}, 1 \mathrm{H}), 9.32(\mathrm{~s}, 1 \mathrm{H}) ;{ }^{13} \mathrm{C}$ NMR $(126 \mathrm{MHz}$, Chloroform-d) $\delta=21.9,24.8,34.1$, 124.7, 125.9, 128.1, 128.2, 128.4, 129.6, 130.0, 130.1, 130.2, 134.0, 136.7, 141.4, 145.5, 147.8, 193.2; HRMS (ESI): calculated for $\mathrm{C}_{24} \mathrm{H}_{24} \mathrm{NO}_{3} \mathrm{~S}[\mathrm{M}+\mathrm{H}]^{+}:$: 406.1471, found 406.1463.<smiles>[3H]N([13CH3])/C(C=O)=C\CCc1ccccc1</smiles>

(Z)-4-methyl-N-(2-morpholinoethyl)-N-(1-oxo-5-phenylpent-2-en-2-yl)benzenesulfonamide (2m)

Synthesized following the general procedure 2.2, using 4-methyl- $N$-(2-morpholinoethyl)- $N$-(4-phenylbut-1-yn-1yl)benzenesulfonamide $1 \mathrm{~m}$ (100 mg, $0.24 \mathrm{mmol}$ ), BiPhePhos as ligand, and $\mathrm{CH}_{2} \mathrm{Cl}_{2} / \mathrm{EtOAc}(80: 20)$ as chromatography eluent. Entitled product $2 \mathrm{~m} \mathrm{(31} \mathrm{mg,} \mathrm{29 \% )} \mathrm{was} \mathrm{obtained} \mathrm{as} \mathrm{yellowish} \mathrm{oil.}$

${ }^{1} \mathrm{H}$ NMR (400 MHz, Chloroform-d) $\delta=2.18-2.26(\mathrm{~m}, 5 \mathrm{H}), 2.37-2.41(\mathrm{~m}, 4 \mathrm{H}), 2.85$ (broad s, 4H), 3.36 (broad s, $1 \mathrm{H}), 3.54-3.57(\mathrm{~m}, 5 \mathrm{H}), 6.84-6.87(\mathrm{~m}, 1 \mathrm{H}), 7.18-7.24(\mathrm{~m}, 3 \mathrm{H}), 7.28-7.33(\mathrm{~m}, 3 \mathrm{H}), 7.65-7.68(\mathrm{~m}, 2 \mathrm{H}), 9.20(\mathrm{~s}$, $1 \mathrm{H}) ;{ }^{13} \mathrm{C}$ NMR (101 MHz, Chloroform-d) $\delta=21.7,31.3,34.2,45.1,53.4,57.4,66.9,126.6,127.8,128.6,128.8$, 129.6, 136.6, 138.8, 140.5, 143.9, 156.6, 189.4; HRMS (ESI): calculated for $\mathrm{C}_{24} \mathrm{H}_{31} \mathrm{~N}_{2} \mathrm{O}_{4} \mathrm{~S}[\mathrm{M}+\mathrm{H}]^{+}$: 443.1999, found 443.1989.<smiles>O=C/C=C\N([13F])CCN1CCOCC1</smiles>

(E)-N-(2-formyl-4-phenylbut-1-en-1-yl)-4-methyl-N-(2-morpholinoethyl)benzenesulfonamide (3m)

Synthesized following the general procedure 2.2, using 4-methyl- $N$-(2-morpholinoethyl)- $N$-(4-phenylbut-1-yn-1yl)benzenesulfonamide $1 \mathrm{~m}$ (93 $\mathrm{mg}, 0.23 \mathrm{mmol}$ ), Xantphos as ligand, and $\mathrm{CH}_{2} \mathrm{Cl}_{2} / \mathrm{EtOAc}$ (80:20) as chromatography eluent. Entitled product $3 \mathrm{~m}(73 \mathrm{mg}, 73 \%)$ was obtained as yellowish oil.

${ }^{1} \mathrm{H}$ NMR (400 MHz, Chloroform-d) $\delta=2.35-2.38(\mathrm{~m}, 4 \mathrm{H}), 2.46-2.49(\mathrm{~m}, 5 \mathrm{H}), 2.58-2.65(\mathrm{~m}, 4 \mathrm{H}), 3.52-3.56$ $(\mathrm{m}, 2 \mathrm{H}), 3.61-3.63(\mathrm{~m}, 4 \mathrm{H}), 7.09-7.18(\mathrm{~m}, 5 \mathrm{H}), 7.37-7.40(\mathrm{~m}, 3 \mathrm{H}), 7.69-7.71(\mathrm{~m}, 2 \mathrm{H}), 9.27(\mathrm{~s}, 1 \mathrm{H}) ;{ }^{13} \mathrm{C} \mathrm{NMR}$ (126 MHz, Chloroform-d) $\delta=21.8,25.2$, 35.5, 44.6, 53.8, 56.6, 66.9, 126.3, 127.4, 128.4, 128.6, 130.4, 135.1, 141.0, 145.4, 147.1, 193.3; HRMS (ESI): calculated for $\mathrm{C}_{24} \mathrm{H}_{31} \mathrm{~N}_{2} \mathrm{O}_{4} \mathrm{~S}[\mathrm{M}+\mathrm{H}]^{+}$: 443.1999, found 443.1993.<smiles>COC(=O)CN([Te])/C(C=O)=C\CCc1ccccc1</smiles>

methyl (Z)-N-(1-oxo-5-phenylpent-2-en-2-yl)- $N$-tosylglycinate (2n)

Synthesized following the general procedure 2.2, using methyl $N$-(4-phenylbut-1-yn-1-yl)- $N$-tosylglycinate 1n (70 $\mathrm{mg}, 0.19 \mathrm{mmol})$, BiPhePhos as ligand, and $\mathrm{CH}_{2} \mathrm{Cl}_{2} /$ EtOAc $(98: 2$ to $97: 3)$ as chromatography eluent. Entitled product $2 \mathrm{n}$ (37 $\mathrm{mg}, 49 \%$ ) was obtained as thick colorless oil.

${ }^{1} \mathrm{H}$ NMR (400 MHz, Chloroform-d) $\delta=2.41(\mathrm{~s}, 3 \mathrm{H}), 2.88(\mathrm{t}, \mathrm{J}=7.6 \mathrm{~Hz}, 2 \mathrm{H}), 3.10$ (large s, 2H), $3.70(\mathrm{~s}, 3 \mathrm{H}), 4.15$ (broad s, 3H), $6.93(\mathrm{t}, J=7.3 \mathrm{~Hz}, 1 \mathrm{H}), 7.21-7.25(\mathrm{~m}, 2 \mathrm{H}), 7.27-7.34(\mathrm{~m}, 5 \mathrm{H}), 7.62-7.64(\mathrm{~m}, 2 \mathrm{H}), 9.15(\mathrm{~s}, 1 \mathrm{H}) ;{ }^{13} \mathrm{C}$ NMR $(101 \mathrm{MHz}$, Chloroform-d) $\delta=21.8,29.9,31.7,33.9,52.4,126.5,127.8,128.7,129.7,136.1,140.6,144.3$, 160.8, 169.3, 189.4; HRMS (ESI): calculated for $\mathrm{C}_{21} \mathrm{H}_{24} \mathrm{NO}_{5} \mathrm{~S}[\mathrm{M}+\mathrm{H}]^{+}:$402.1370, found 402.1365 . 
<smiles>CC(=O)CN(C=CC(=O)c1ccccc1)CCc1ccccc1</smiles>

methyl (E)- $N$-(2-formyl-4-phenylbut-1-en-1-yl)- $N$-tosylglycinate (3n)

Synthesized following the general procedure 2.2, using methyl $N$-(4-phenylbut-1-yn-1-yl)- $N$-tosylglycinate 1 n (70 $\mathrm{mg}, 0.19 \mathrm{mmol})$, BiPhePhos as ligand, and $\mathrm{CH}_{2} \mathrm{Cl}_{2} / \mathrm{EtOAc}(98: 2$ to $97: 3)$ as chromatography eluent. Entitled product 3n (39 $\mathrm{mg}, 51 \%)$ was obtained as thick yellowish oil.

${ }^{1} \mathrm{H}$ NMR $(400 \mathrm{MHz}$, Chloroform-d) $\delta=2.37-2.41(\mathrm{~m}, 2 \mathrm{H}), 2.47(\mathrm{~s}, 3 \mathrm{H}), 2.58-2.62(\mathrm{~m}, 2 \mathrm{H}), 3.62(\mathrm{~s}, 3 \mathrm{H}), 4.16(\mathrm{~s}$, $2 \mathrm{H}), 7.05-7.08(\mathrm{~m}, 2 \mathrm{H}), 7.12-7.16(\mathrm{~m}, 1 \mathrm{H}), 7.17-7.21(\mathrm{~m}, 2 \mathrm{H}), 7.36-7.38(\mathrm{~m}, 2 \mathrm{H}), 7.45(\mathrm{~s}, 1 \mathrm{H}), 7.71-7.73$ $(\mathrm{m}, 2 \mathrm{H}), 9.26(\mathrm{~s}, 1 \mathrm{H}) ;{ }^{13} \mathrm{C}$ NMR $(101 \mathrm{MHz}$, Chlo roform-d) $\delta=21.8,25.5,35.2,47.7,52.9,123.7,126.4,127.8$, 128.5, 128.6, 130.3, 134.8, 140.9, 145.6, 146.9, 167.7, 193.1; HRMS (ESI): calculated for $\mathrm{C}_{21} \mathrm{H}_{24} \mathrm{NO}_{5} \mathrm{~S}[\mathrm{M}+\mathrm{H}]^{+}$: 402.1370, found 402.1363 .

(Z)-2-(2-oxooxazolidin-3-yl)-3-phenylacrylaldehyde (20)<smiles>O=C/C(=C/c1ccccc1)N1CCOC1=O</smiles>

Synthesized following the general procedure 2.2, 3-(phenylethynyl)oxazolidin-2-one 10 (88 $\mathrm{mg}, 0.47 \mathrm{mmol}$ ), Xantphos as ligand, and pentane $/ \mathrm{CH}_{2} \mathrm{Cl}_{2} / \mathrm{Et}_{2} \mathrm{O}$ (35:50:15) as chromatography eluent. Entitled product 20 (23 mg, $23 \%)$ was obtained as colorless oil.

${ }^{1} \mathrm{H}$ NMR (400 MHz, Chloroform-d) $\delta=3.78(\mathrm{~m}, 2 \mathrm{H}), 4.55(\mathrm{~m}, 2 \mathrm{H}), 7.41(\mathrm{~s}, 1 \mathrm{H}), 7.44-7.49(\mathrm{~m}, 3 \mathrm{H}), 7.62-7.67(\mathrm{~m}$, 2H), $9.54(\mathrm{~s}, 1 \mathrm{H}) ;{ }^{13} \mathrm{C}$ NMR (101 MHz, Chloroform-d) $\delta=45.2,63.3,129.3,130.4,131.8,132.2,134.8,148.2,157.5$, 189.1; HRMS (ESI): calculated for $\mathrm{C}_{12} \mathrm{H}_{11} \mathrm{KNO}_{3}[\mathrm{M}+\mathrm{K}]^{+}:$: 256.0376, found 256.0371 .<smiles></smiles>

(E)-3-(2-oxooxazolidin-3-yl)-2-phenylacrylaldehyde (3o)

Synthesized following the general procedure 2.2, 3-(phenylethynyl)oxazolidin-2-one 10 (70 $\mathrm{mg}, 0.37 \mathrm{mmol}$ ), Xantphos as ligand, and pentane $/ \mathrm{CH}_{2} \mathrm{Cl}_{2} / \mathrm{Et}_{2} \mathrm{O}$ (35:50:15) as chromatography eluent. Entitled product 30 (33 mg, $41 \%)$ was obtained as colorless oil.

${ }^{1} \mathrm{H}$ NMR $(400 \mathrm{MHz}$, Chloroform-d) $\delta=3.29(\mathrm{~m}, 2 \mathrm{H}), 4.29(\mathrm{~m}, 2 \mathrm{H}), 7.15-7.20(\mathrm{~m}, 2 \mathrm{H}) 7.36-7.41(\mathrm{~m}, 3 \mathrm{H}), 7.66(\mathrm{~s}$, 1H), 9.55 (s, 1H); ${ }^{13} \mathrm{C}$ NMR (101 MHz, Chloroform-d) $\delta=44.6,63.1,125.5,128.2,128.7,130.9,131.2,143.1,155.6$, 191.5; HRMS (ESI): calculated for $\mathrm{C}_{12} \mathrm{H}_{11} \mathrm{KNO}_{3}[\mathrm{M}+\mathrm{K}]^{+}: 256.0376$, found 256.0374 .<smiles>O=C/C(=C/C1CCCCC1)N1CCOC1=O</smiles>

(Z)-3-cyclohexyl-2-(2-oxooxazolidin-3-yl)acrylaldehyde (2p)

Synthesized following the general procedure 2.2, 3-(cyclohexylethynyl)oxazolidin-2-one 1p (120 mg, 0.62 $\mathrm{mmol}), \mathrm{BiPhePhos}$ as ligand, and pentane $/ \mathrm{CH}_{2} \mathrm{Cl}_{2} / \mathrm{Et}_{2} \mathrm{O}(40: 50: 10)$ as chromatography eluent. Entitled product $\mathbf{2 p}$ (65 mg, 47\%) was obtained as amorphous white solid. 
${ }^{1} \mathrm{H}$ NMR (400 MHz, Chloroform- $\left.d\right) \delta=1.16-1.38(\mathrm{~m}, 5 \mathrm{H}), 1.66-1.81(\mathrm{~m}, 5 \mathrm{H}), 2.54$ (qt, $\left.J=10.7,3.3 \mathrm{~Hz}, 1 \mathrm{H}\right), 3.79$ $(\mathrm{m}, 2 \mathrm{H}), 4.47(\mathrm{~m}, 2 \mathrm{H}), 6.59(\mathrm{~d}, J=10.2 \mathrm{~Hz}, 1 \mathrm{H}), 9.32(\mathrm{~s}, 1 \mathrm{H}) ;{ }^{13} \mathrm{C}$ NMR $\left(101 \mathrm{MHz}\right.$, Chloroform-d) $\delta=25.2\left(2^{*} \mathrm{C}\right)$, 25.7, $31.5(2 * \mathrm{C}), 38.0,46.0,62.9,135.5,156.9,160.5,189.3$; HRMS (ESI): calculated for $\mathrm{C}_{12} \mathrm{H}_{17} \mathrm{NNaO}_{3}[\mathrm{M}+\mathrm{Na}]^{+}$: 246.1106 , found 246.1101 .<smiles>O=C/C=C\N1CCOC1=O</smiles>

(E)-2-cyclohexyl-3-(2-oxooxazolidin-3-yl)acrylaldehyde (3p)

Synthesized following the general procedure 2.2, 3-(cyclohexylethynyl)oxazolidin-2-one 1p (120 mg, 0.62 $\mathrm{mmol})$, Xantphos as ligand, and pentane $/ \mathrm{CH}_{2} \mathrm{Cl}_{2} / \mathrm{Et}_{2} \mathrm{O}$ (40:50:10) as chromatography eluent. Entitled product 3p (41 mg, 29\%) was obtained as thick yellowish oil.

${ }^{1} \mathrm{H}$ NMR (400 MHz, Chloroform- $\left.d\right) \delta=1.12-1.35(\mathrm{~m}, 3 \mathrm{H}), 1.46(\mathrm{~m}, 2 \mathrm{H}), 1.66(\mathrm{~m}, 1 \mathrm{H}), 1.81(\mathrm{~m}, 2 \mathrm{H}), 2.09(\mathrm{qd}, J=$ 12.6, 3.4 Hz, 2H), $2.48-2.57(\mathrm{~m}, 1 \mathrm{H}), 4.21(\mathrm{~m}, 2 \mathrm{H}), 4.53(\mathrm{~m}, 2 \mathrm{H}), 7.23(\mathrm{~s}, 1 \mathrm{H}), 9.27(\mathrm{~d}, J=2.2 \mathrm{~Hz}, 1 \mathrm{H}) ;{ }^{13} \mathrm{C} \mathrm{NMR}$ (101 MHz, Chloroform-d) $\delta=25.7,27.1(2 * \mathrm{C}), 30.3(2 * \mathrm{C}), 37.1,44.7,62.7,129.3,143.5,155.9,193.9$; HRMS (ESI): calculated for $\mathrm{C}_{12} \mathrm{H}_{17} \mathrm{NNaO}_{3}[\mathrm{M}+\mathrm{Na}]^{+}:$246.1106, found 246.1106 .<smiles>CC(C)(C)/C=C\C=O</smiles>

(Z)-4,4-dimethyl-2-(2-oxooxazolidin-3-yl)pent-2-enal (2q)

Synthesized following the general procedure 2.2, 3-(3,3-dimethylbut-1-yn-1-yl)oxazolidin-2-one 1q (78 mg, 0.47 $\mathrm{mmol})$, Xantphos as ligand, and pentane/ $\mathrm{CH}_{2} \mathrm{Cl}_{2} / \mathrm{Et}_{2} \mathrm{O}$ (35:50:15) as chromatography eluent. Entitled product 2q (56 mg, 61\%) was obtained as amorphous white solid.

${ }^{1} \mathrm{H}$ NMR $(400 \mathrm{MHz}$, Chloroform-d) $\delta=1.23(\mathrm{~s}, 9 \mathrm{H}), 3.32-4.06(\mathrm{bm}, 2 \mathrm{H}), 4.43(\mathrm{~m}, 2 \mathrm{H}), 6.72(\mathrm{~s}, 1 \mathrm{H}), 9.28(\mathrm{~s}, 1 \mathrm{H})$; ${ }^{13} \mathrm{C}$ NMR $\left(101 \mathrm{MHz}\right.$, Chloroform-d) $\delta=29.1\left(3^{*} \mathrm{C}\right), 35.5,46.7,62.9,135.2,157.6,166.0,190.1$; HRMS (ESI): calculated for $\mathrm{C}_{10} \mathrm{H}_{15} \mathrm{KNO}_{3}[\mathrm{M}+\mathrm{K}]^{+}:$236.0689, found 236.0633 . 
3. ${ }^{1} \mathrm{H} \&{ }^{13} \mathrm{C}$ NMR spectra 
${ }^{1} \mathrm{H}$ NMR of compound $\mathbf{1 a}\left(400 \mathrm{MHz}, \mathrm{CDCl}_{3}\right)$ :

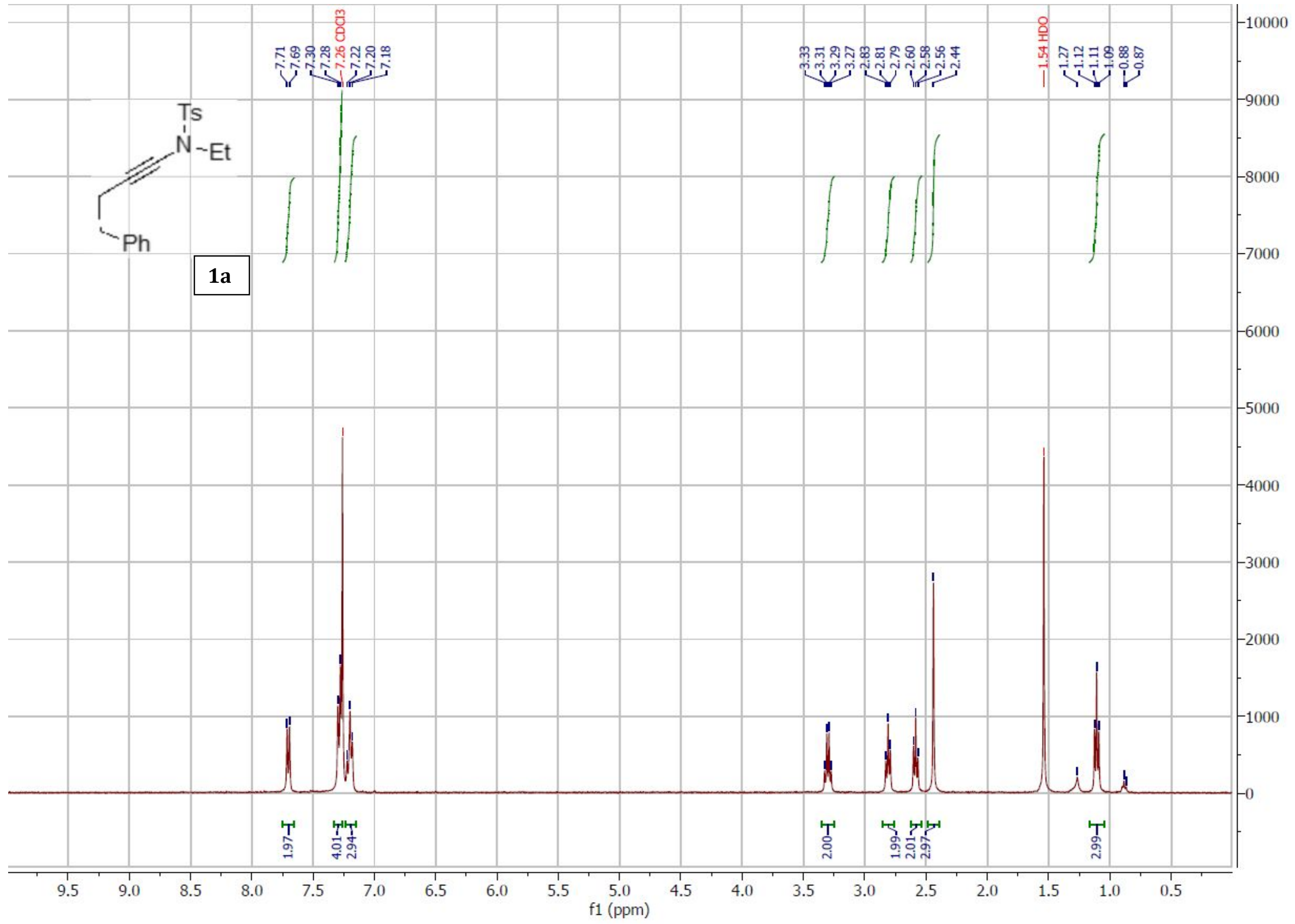


${ }^{13} \mathrm{C}$ NMR of compound $\mathbf{1 a}\left(101 \mathrm{MHz}, \mathrm{CDCl}_{3}\right)$ :

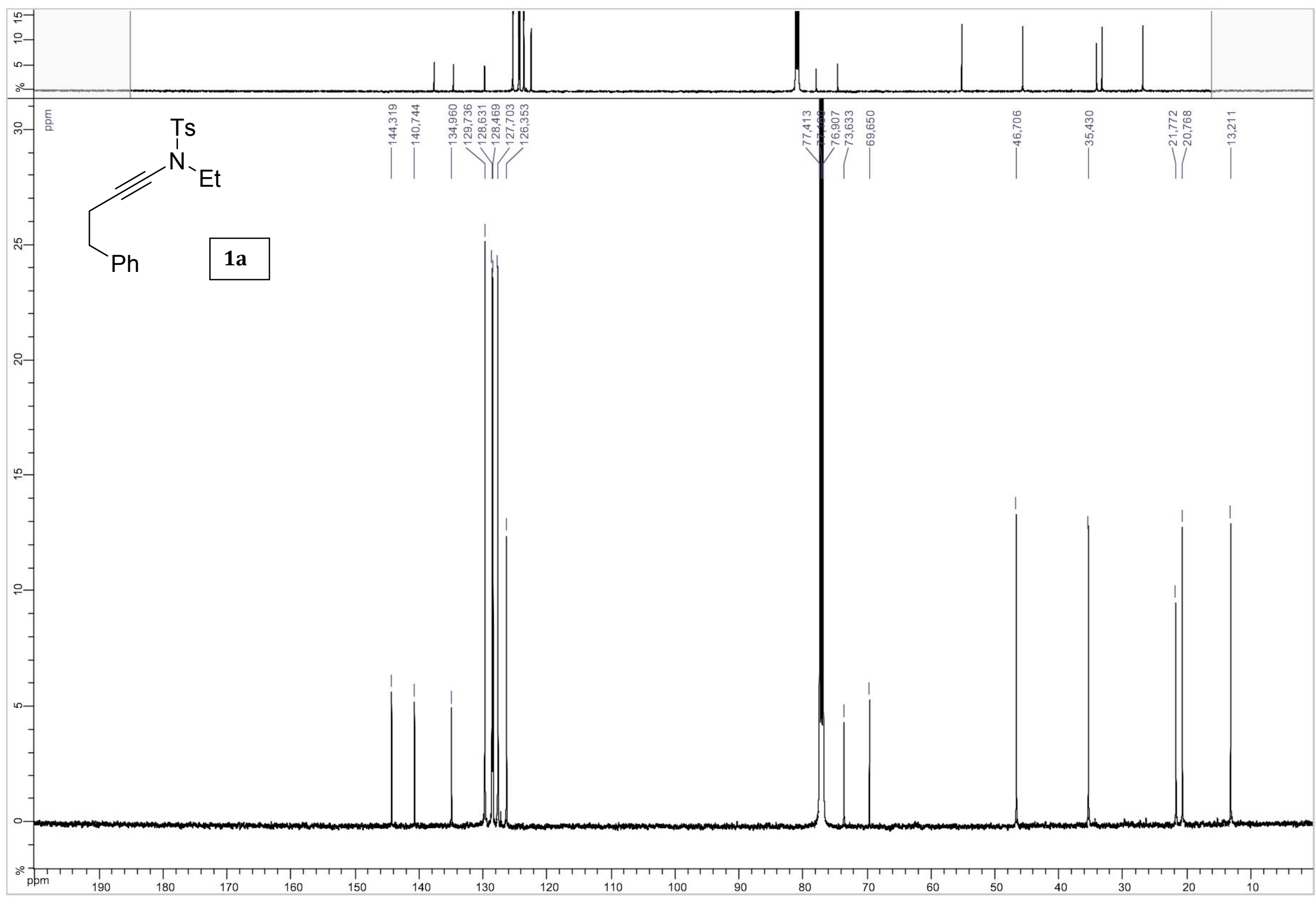


${ }^{1} \mathrm{H}$ NMR of compound $\mathbf{1 b}\left(400 \mathrm{MHz}, \mathrm{CDCl}_{3}\right)$ :

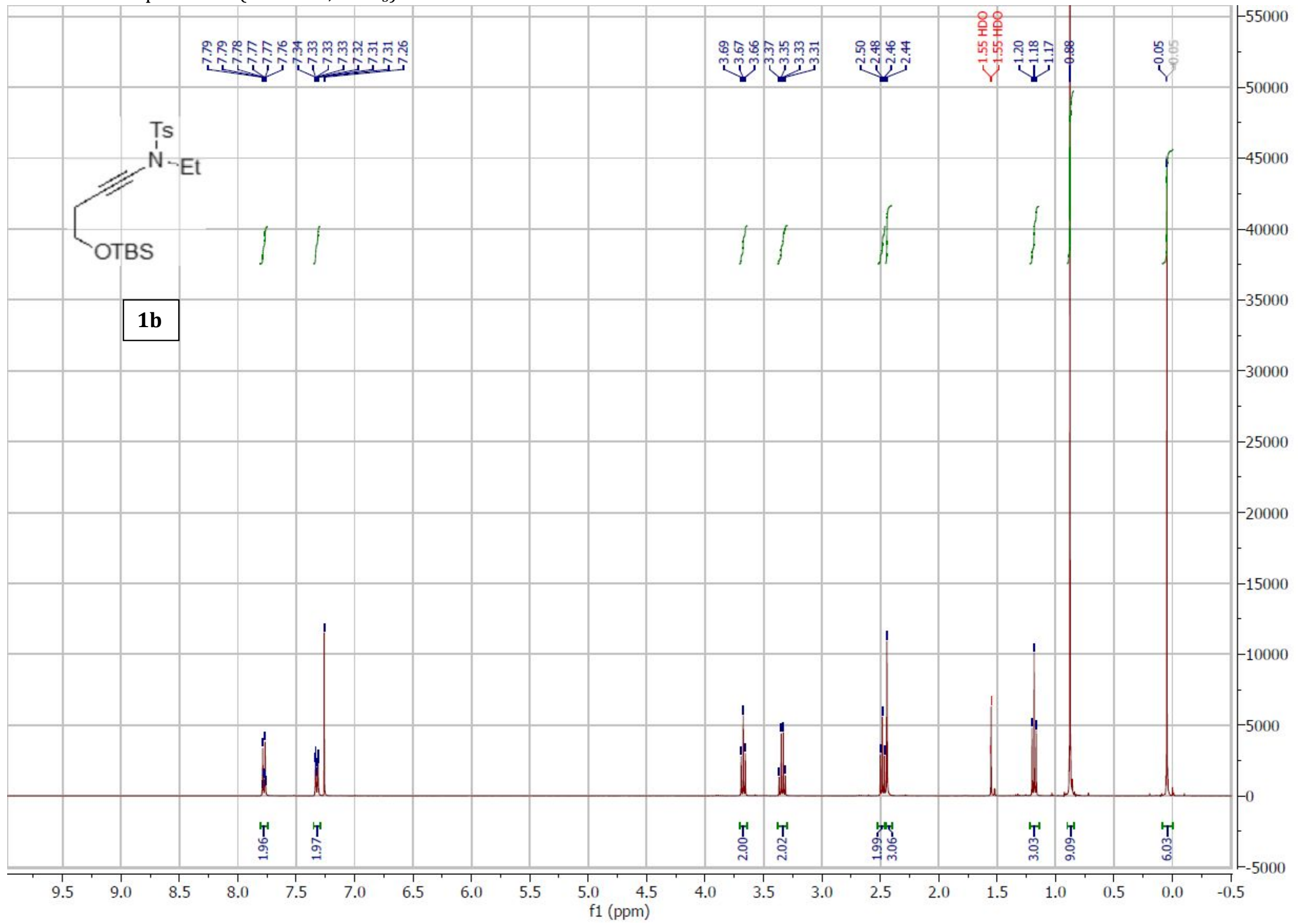


${ }^{13} \mathrm{C}$ NMR of compound $\mathbf{1 b}\left(101 \mathrm{MHz}, \mathrm{CDCl}_{3}\right)$ :

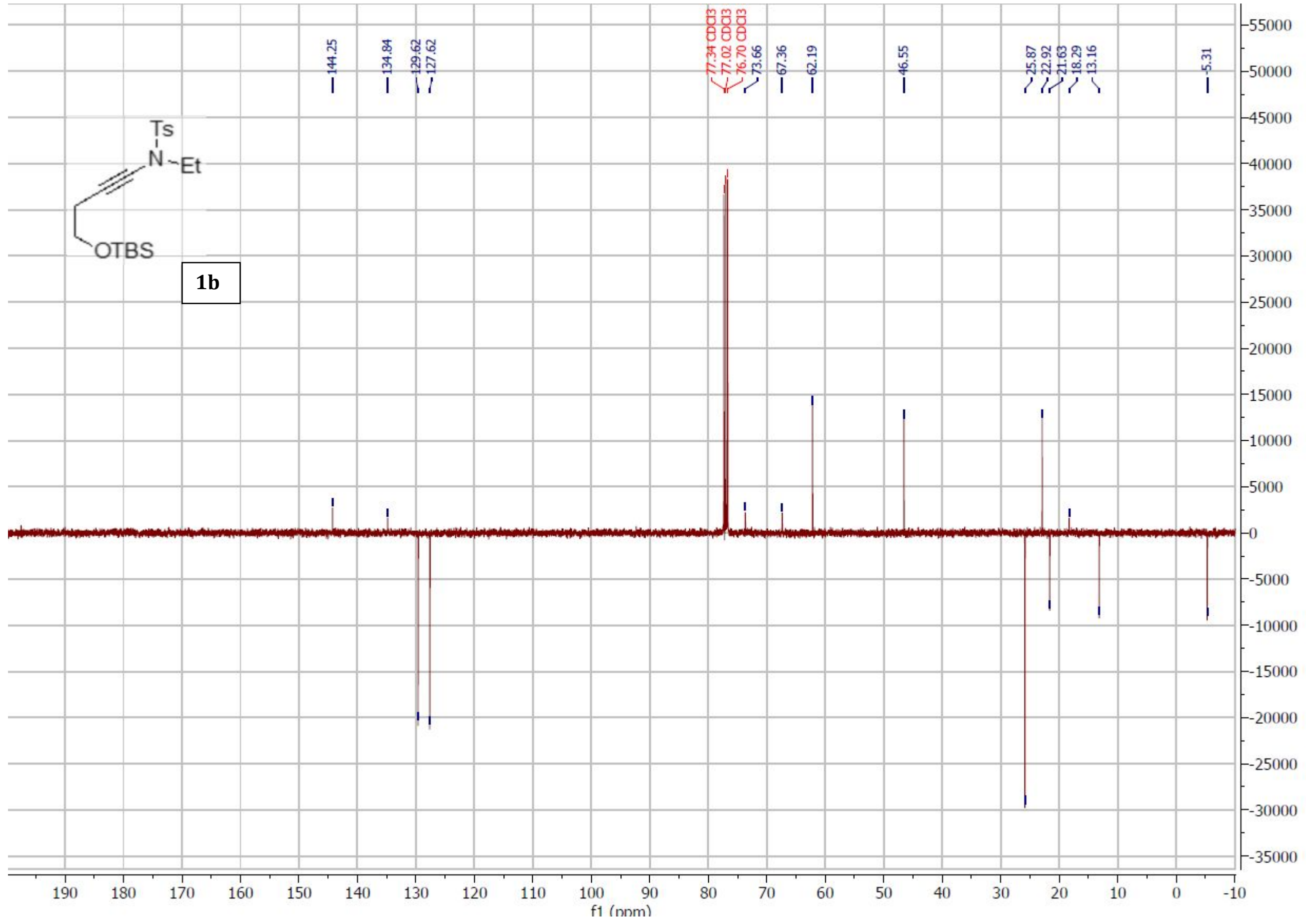


${ }^{1} \mathrm{H}$ NMR of compound $\mathbf{1 c}\left(500 \mathrm{MHz}, \mathrm{CDCl}_{3}\right)$ :

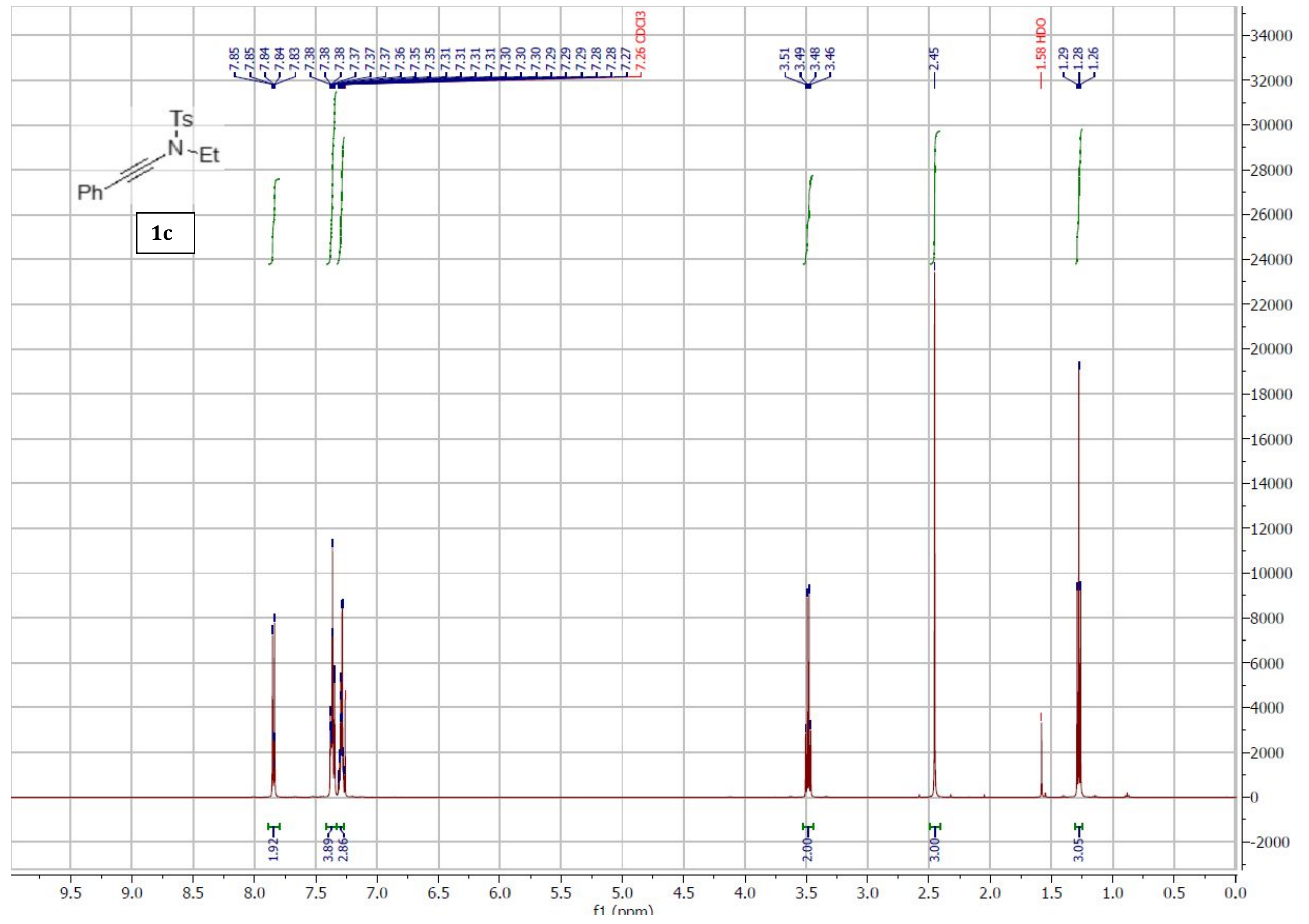


${ }^{13} \mathrm{C}$ NMR of compound $1 \mathrm{c}\left(126 \mathrm{MHz}, \mathrm{CDCl}_{3}\right)$ :

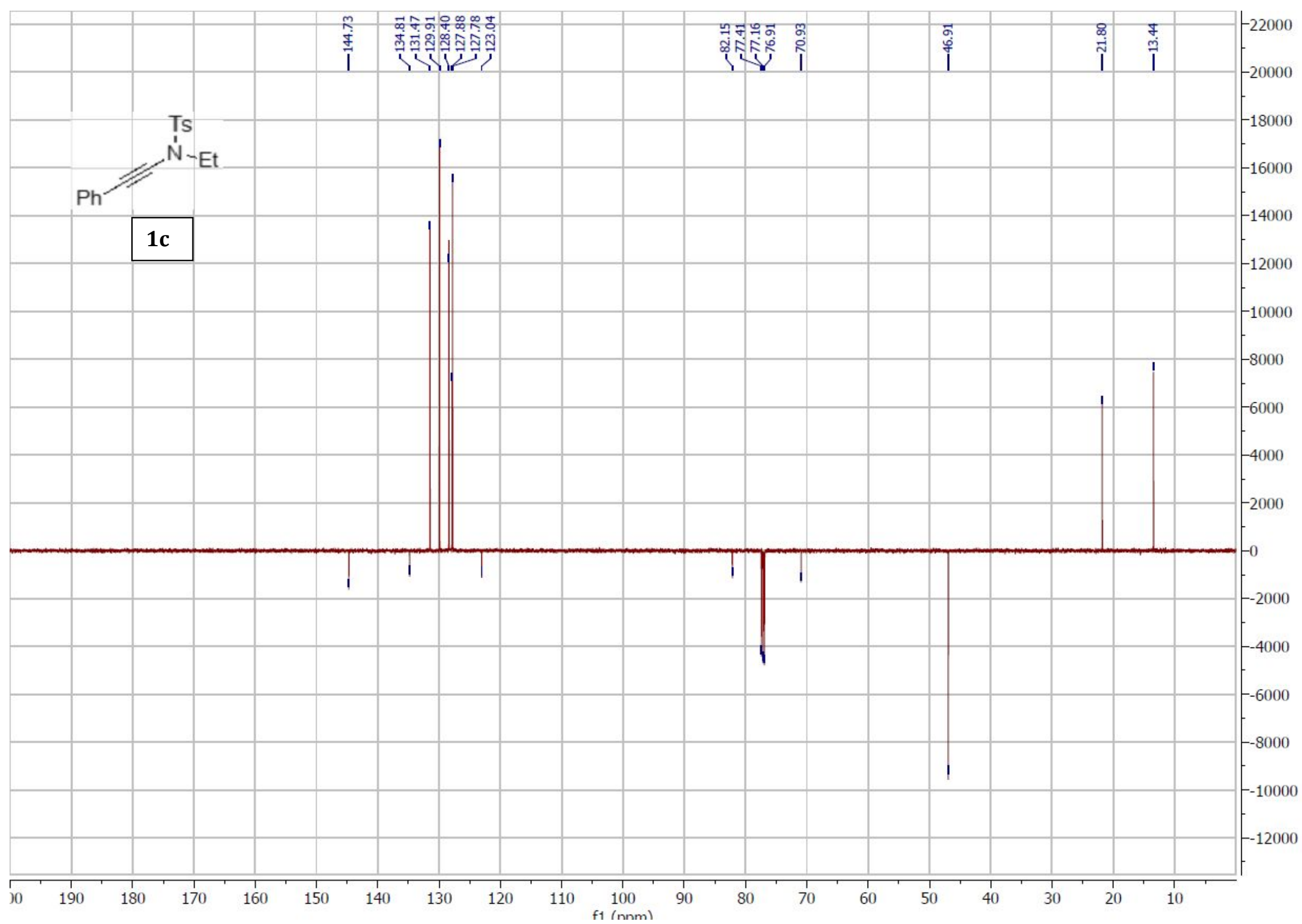


${ }^{1} \mathrm{H}$ NMR of compound $\mathbf{1 d}\left(400 \mathrm{MHz}, \mathrm{CDCl}_{3}\right)$ :

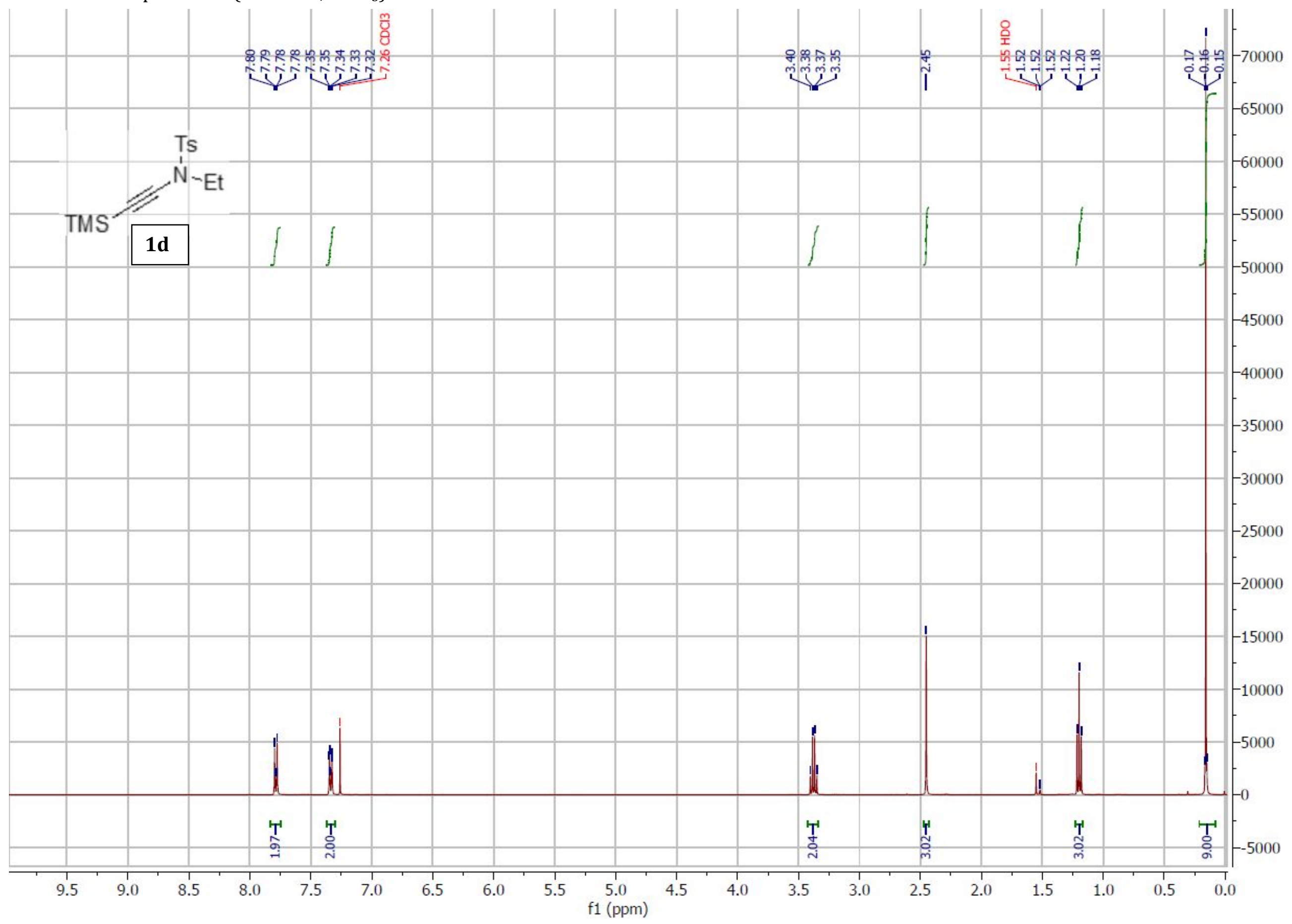


${ }^{13} \mathrm{C}$ NMR of compound $\mathbf{1 d}\left(101 \mathrm{MHz}, \mathrm{CDCl}_{3}\right)$ :

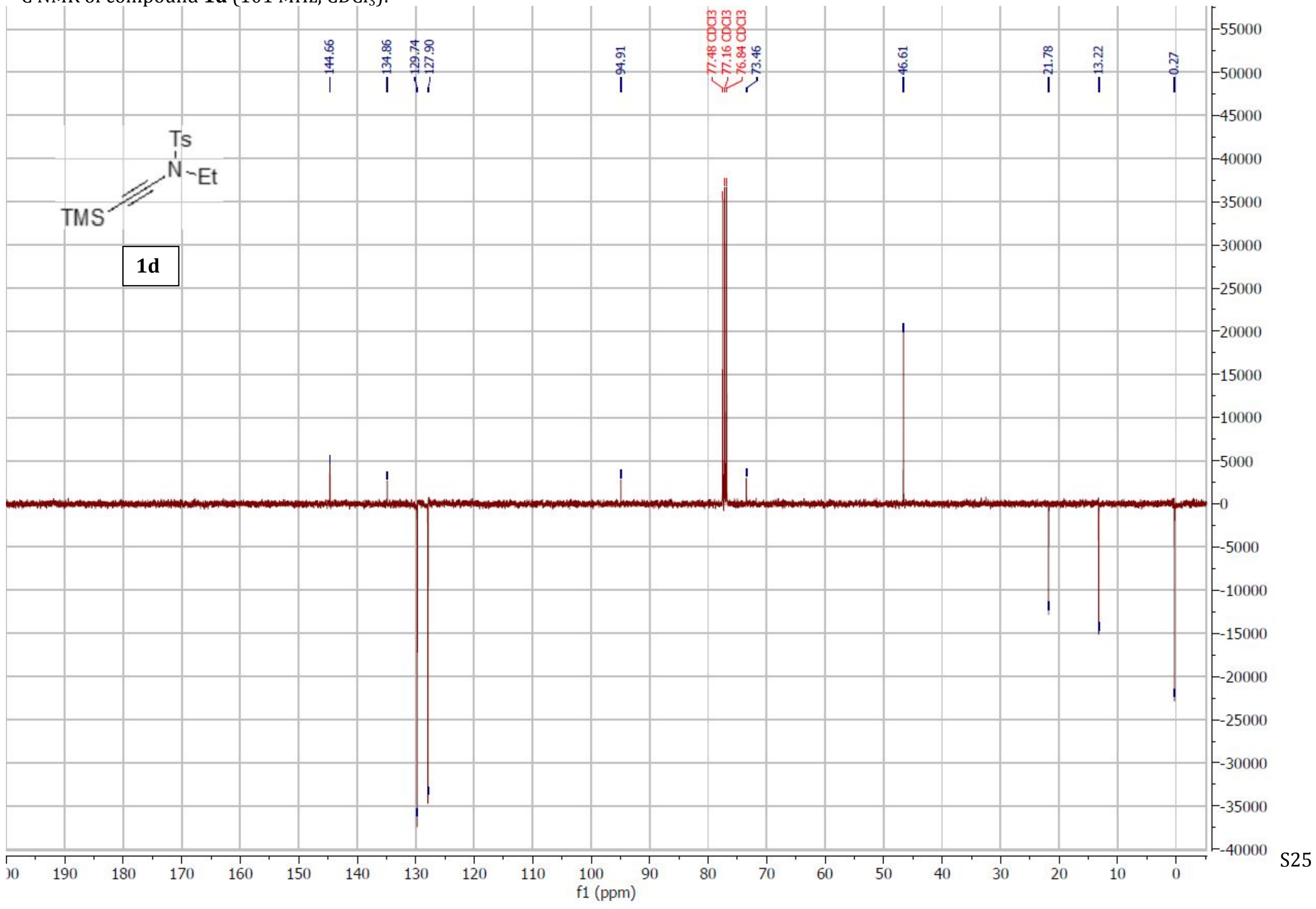


${ }^{1} \mathrm{H}$ NMR of compound $\mathbf{1 e}\left(400 \mathrm{MHz}, \mathrm{CDCl}_{3}\right)$ :

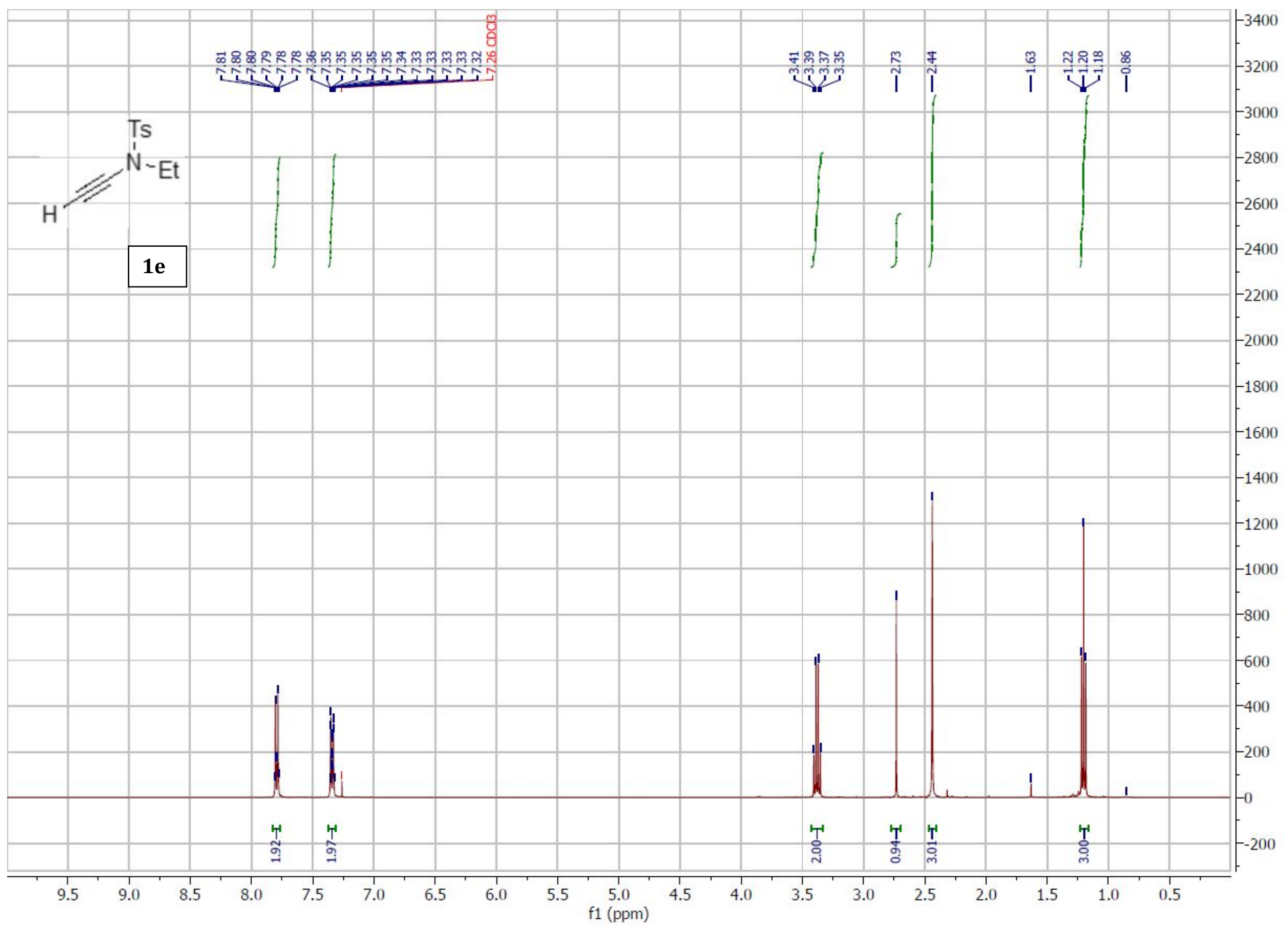


${ }^{13} \mathrm{C}$ NMR of compound $1 \mathbf{e}\left(101 \mathrm{MHz}, \mathrm{CDCl}_{3}\right)$ :

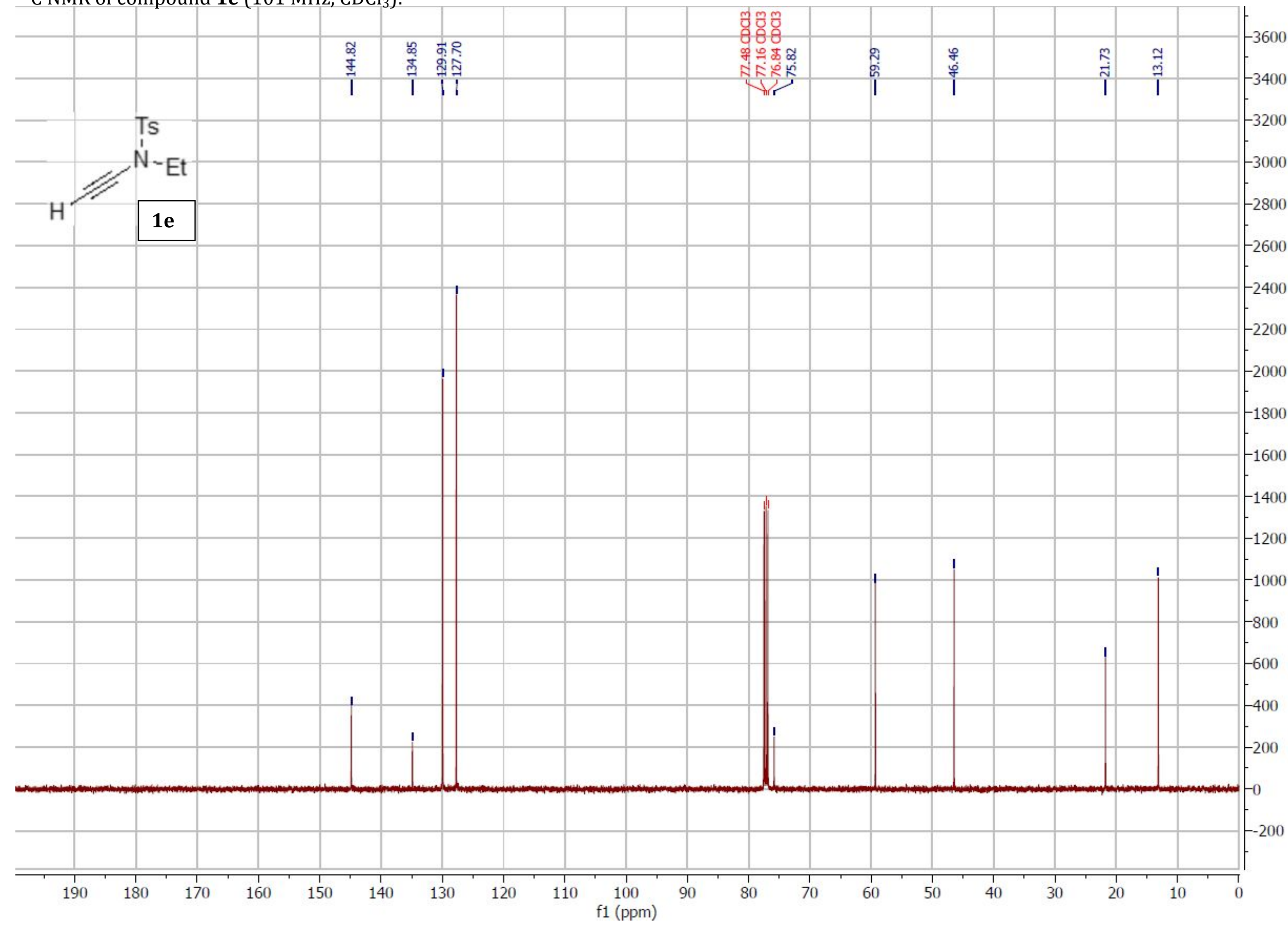


${ }^{1} \mathrm{H}$ NMR of compound $\mathbf{1 f}\left(400 \mathrm{MHz}, \mathrm{CDCl}_{3}\right)$ :

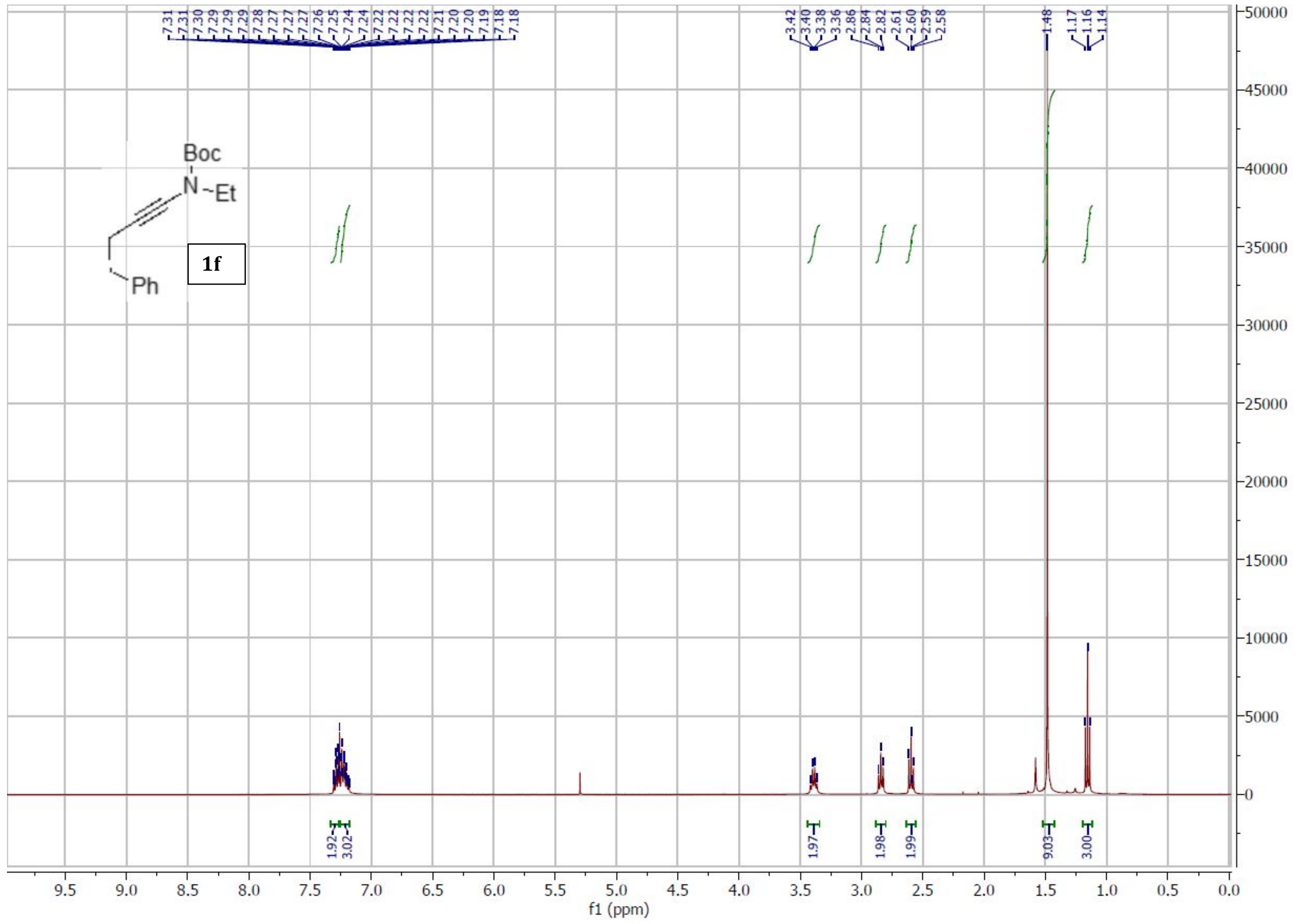


${ }^{13} \mathrm{C}$ NMR of compound $\mathbf{1 f}\left(126 \mathrm{MHz}, \mathrm{CDCl}_{3}\right)$ :

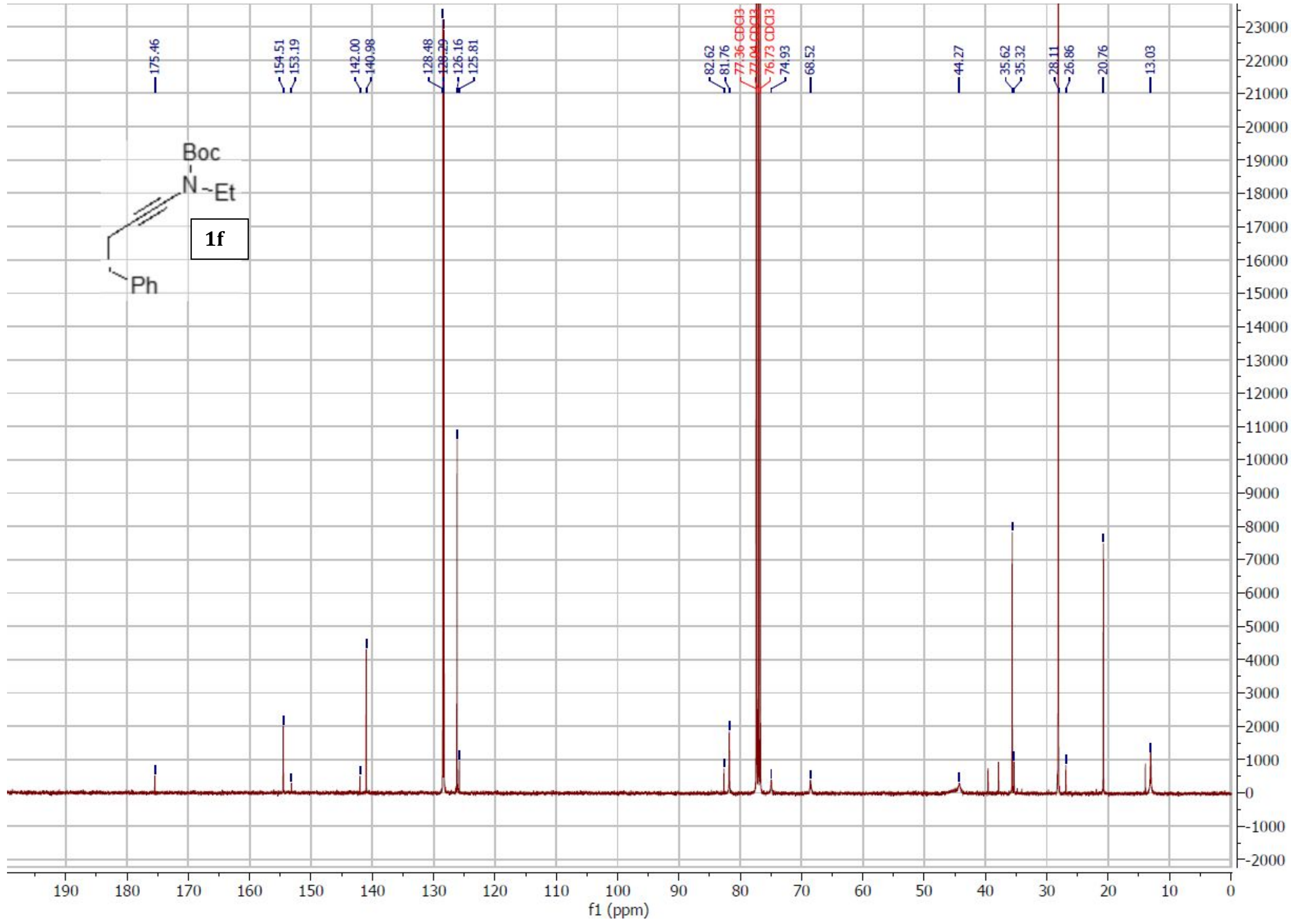


${ }^{1} \mathrm{H}$ NMR of compound $\mathbf{1 g}$ (400 MHz, $\mathrm{CDCl}_{3}$ ):

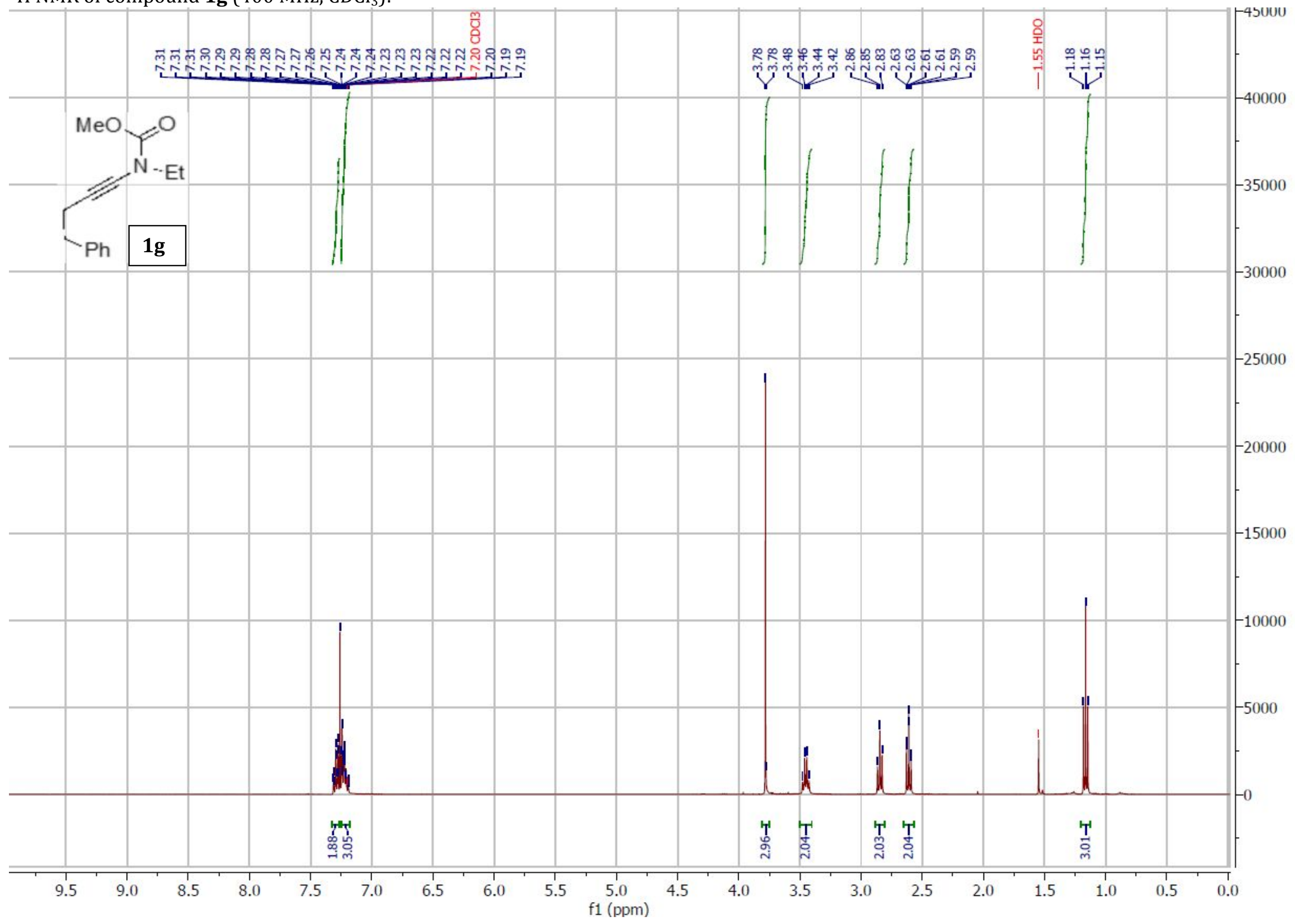


${ }^{13} \mathrm{C}$ NMR of compound 1 g (126 MHz, $\left.\mathrm{CDCl}_{3}\right)$ :

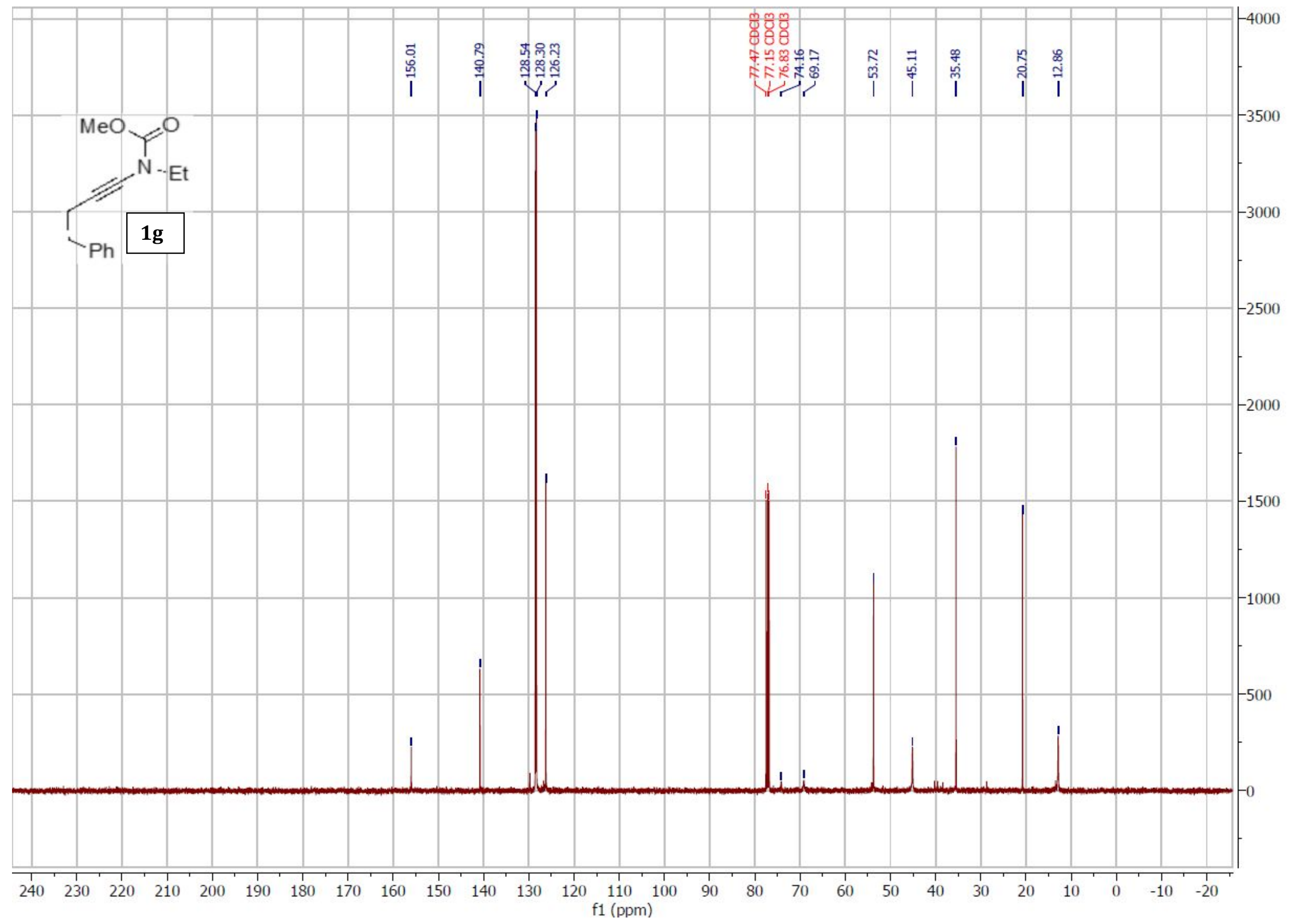


${ }^{1} \mathrm{H}$ NMR of compound $\mathbf{1 h}\left(400 \mathrm{MHz}, \mathrm{CDCl}_{3}\right)$ :

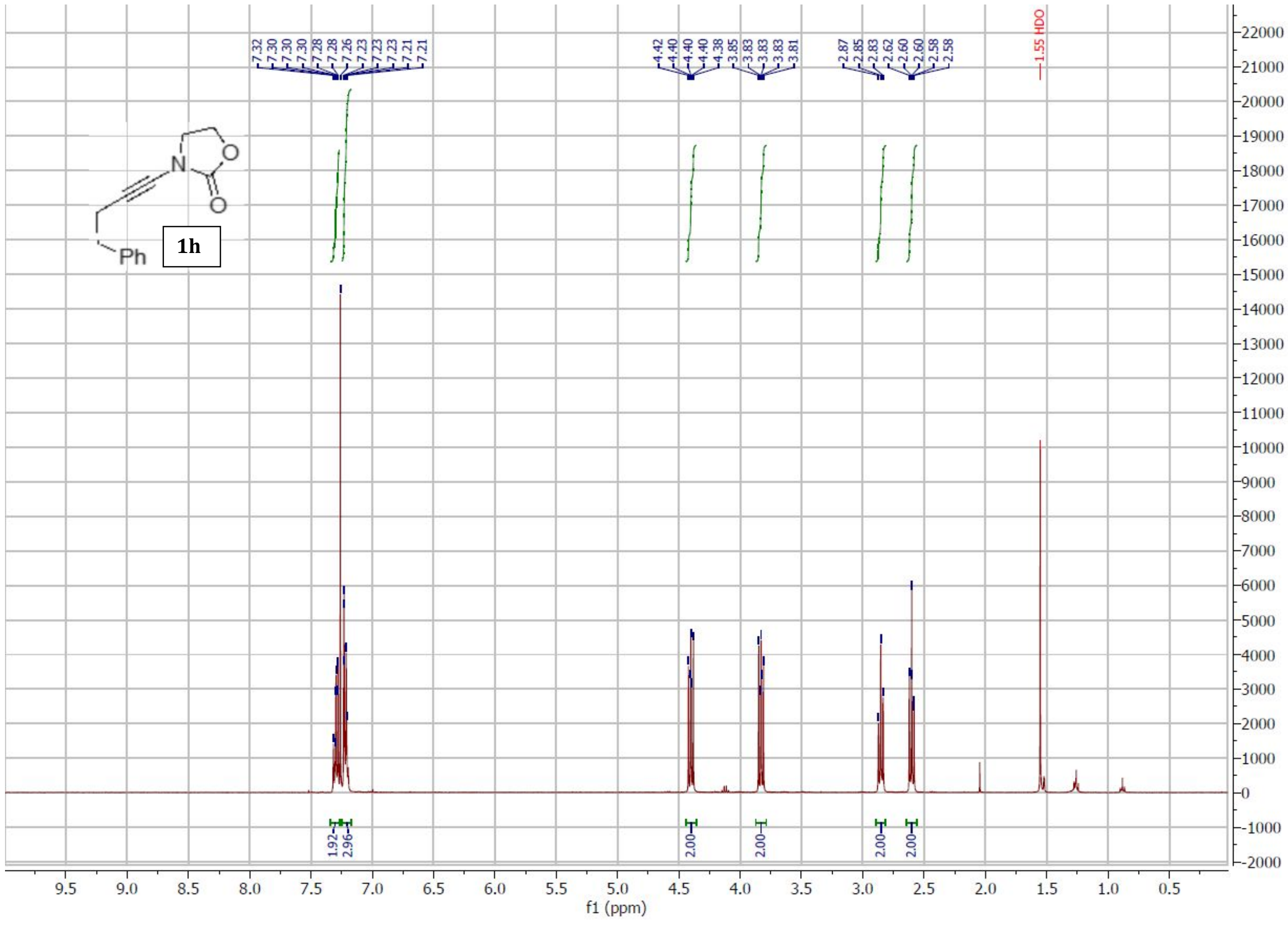


${ }^{1} \mathrm{H}$ NMR of compound $\mathbf{1 i}\left(400 \mathrm{MHz}, \mathrm{CDCl}_{3}\right.$ ):

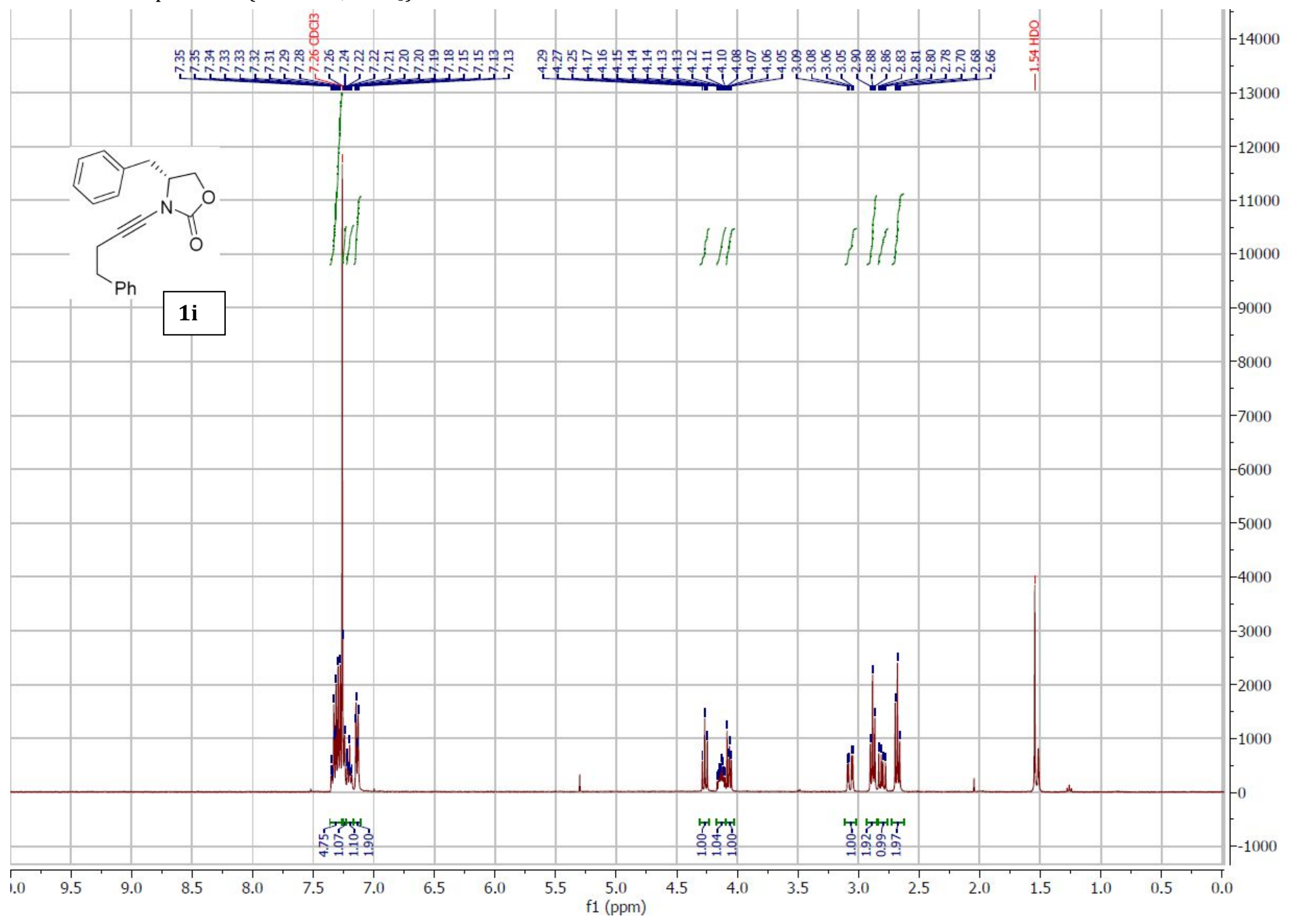


${ }^{1} \mathrm{H}$ NMR of compound $\mathbf{1 j}\left(500 \mathrm{MHz}, \mathrm{CDCl}_{3}\right)$ :

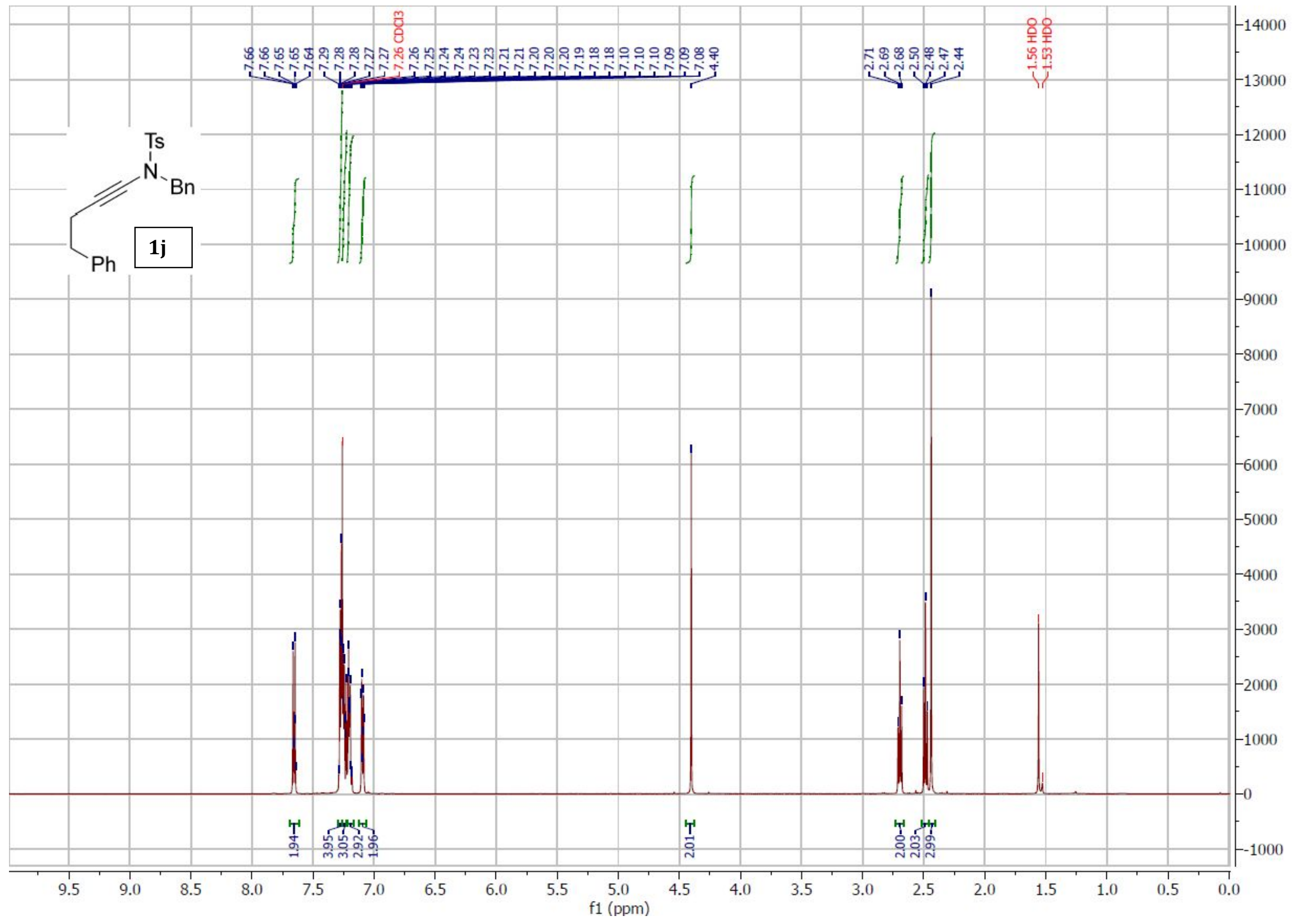


${ }^{1} \mathrm{H}$ NMR of compound $\mathbf{1 k}\left(400 \mathrm{MHz}, \mathrm{CDCl}_{3}\right)$ :

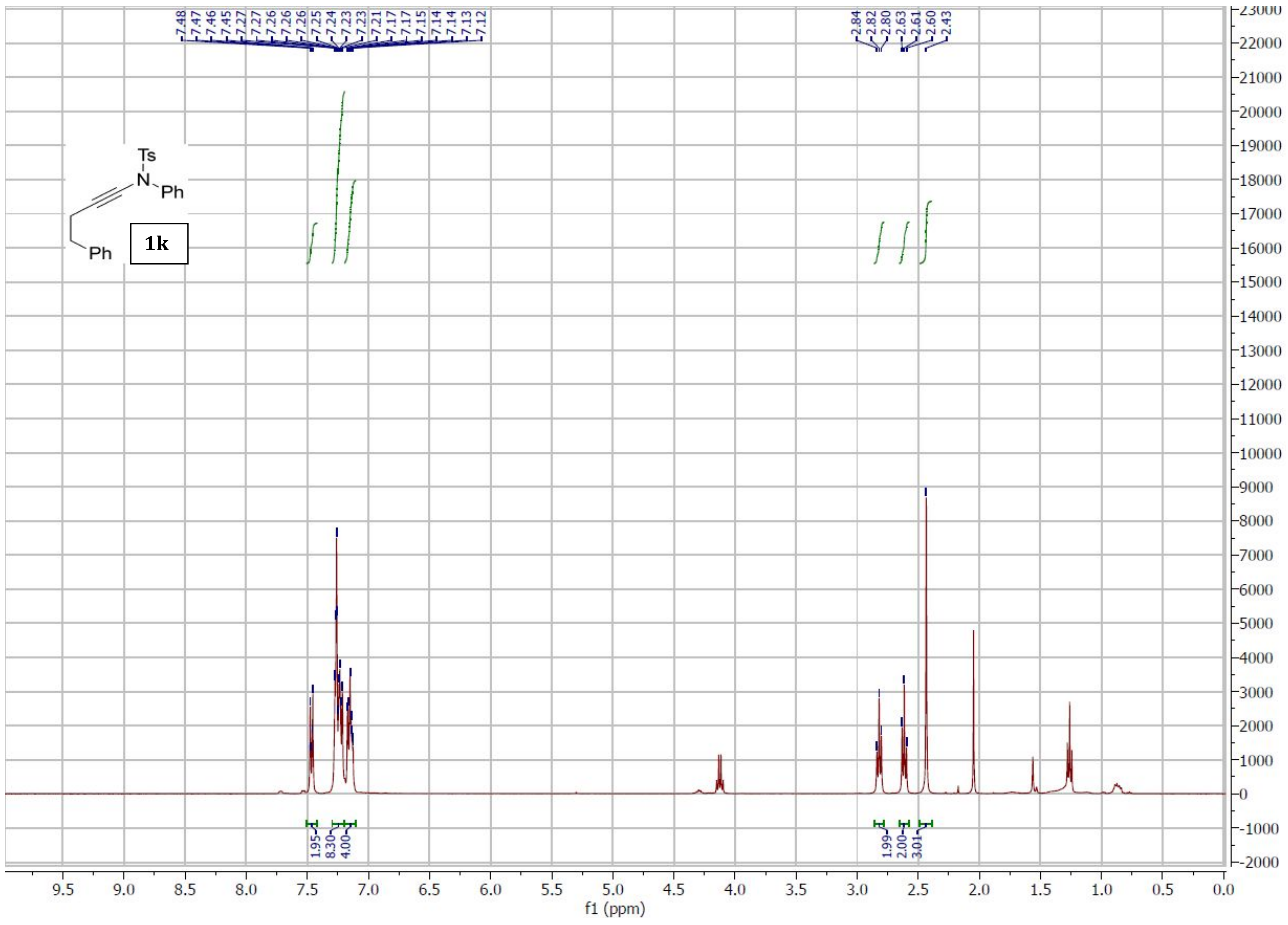


${ }^{1} \mathrm{H}$ NMR of compound 11 (400 MHz, $\mathrm{CDCl}_{3}$ ):

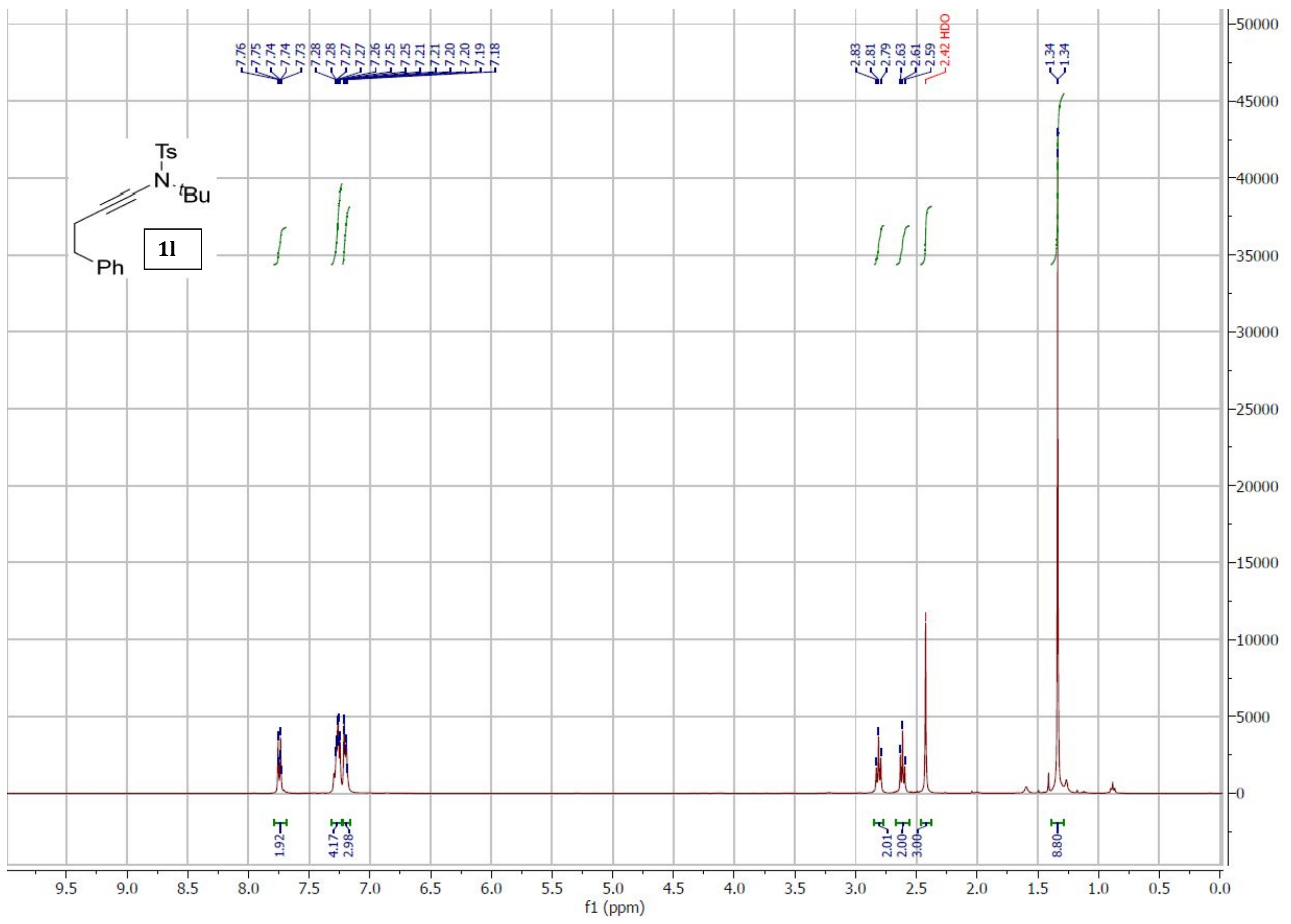


${ }^{13} \mathrm{C}$ NMR of compound 11 (101 MHz, $\left.\mathrm{CDCl}_{3}\right)$ :

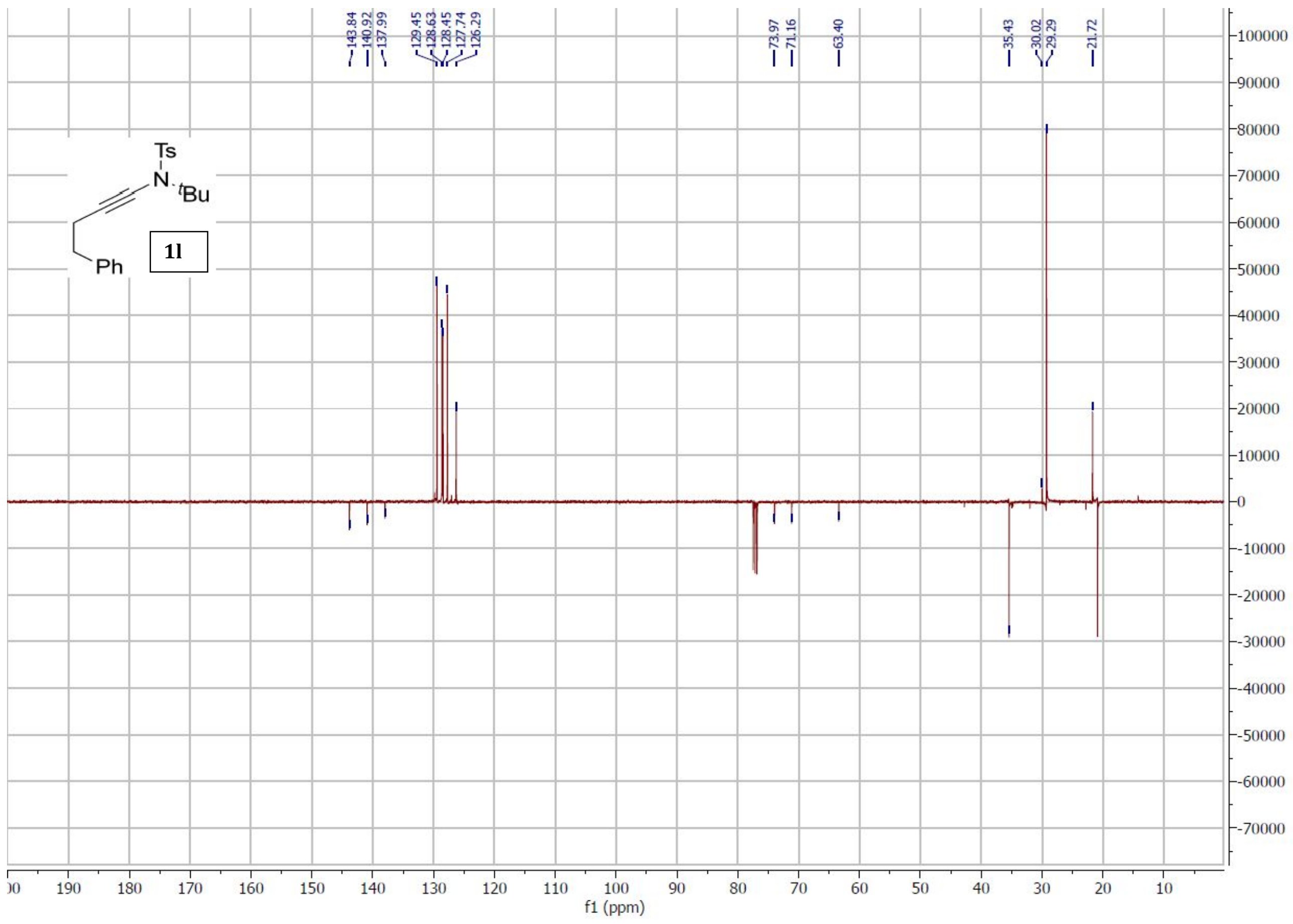


${ }^{1} \mathrm{H}$ NMR of compound $\mathbf{1 m}\left(400 \mathrm{MHz}, \mathrm{CDCl}_{3}\right)$ :

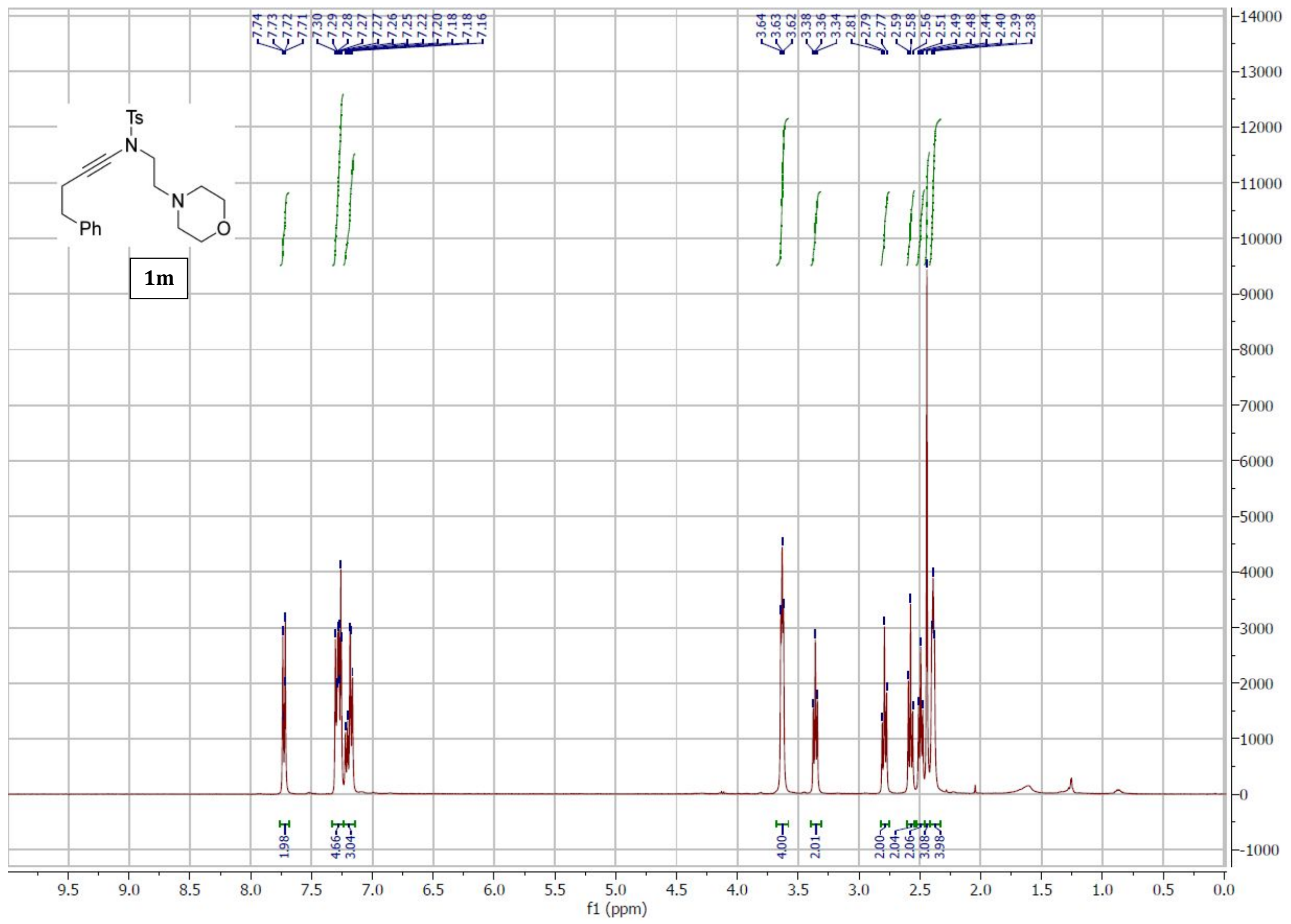


${ }^{13} \mathrm{C}$ NMR of compound $\mathbf{1 m}\left(101 \mathrm{MHz}, \mathrm{CDCl}_{3}\right)$ :

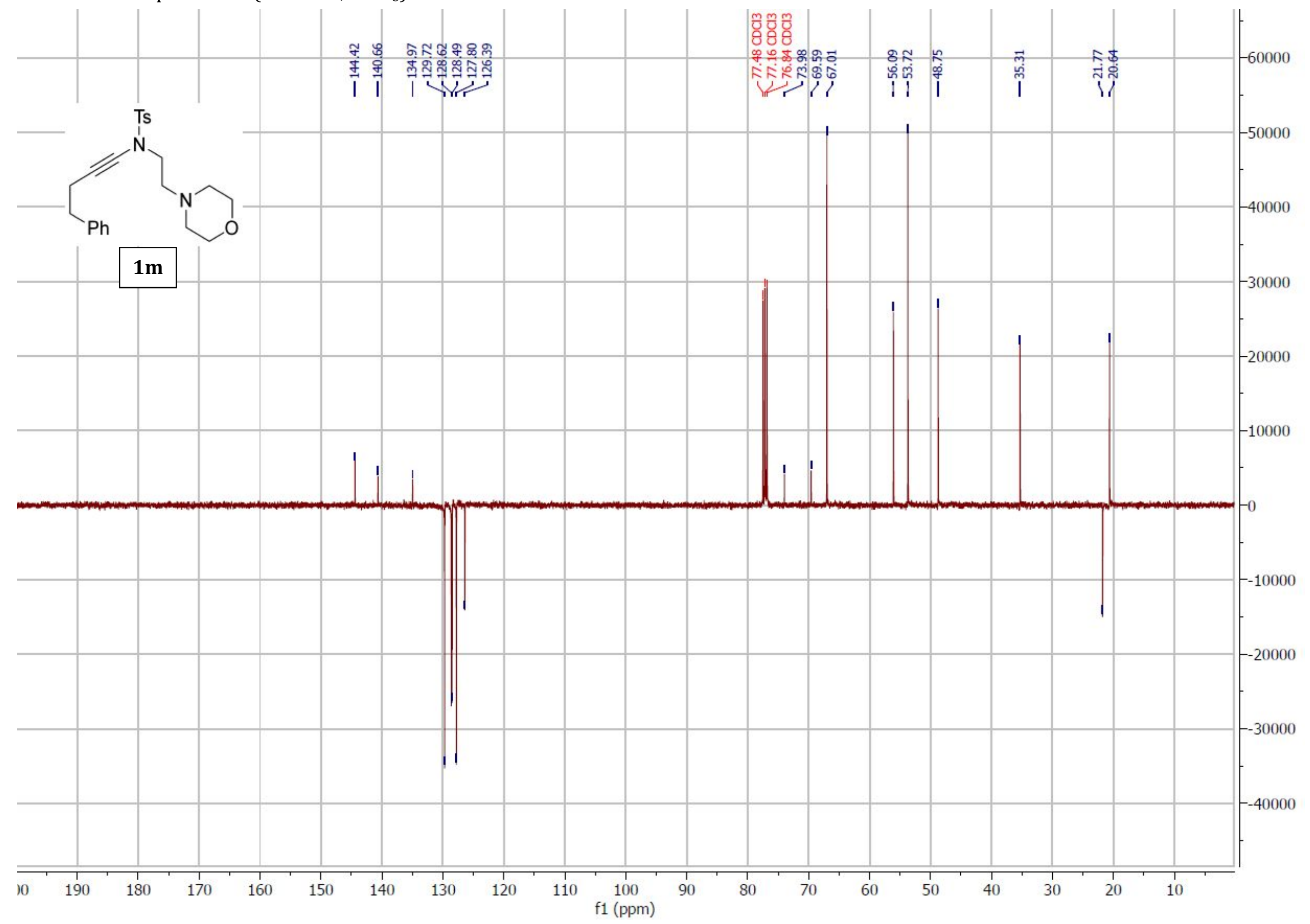


${ }^{1} \mathrm{H}$ NMR of compound $\mathbf{1 n}\left(400 \mathrm{MHz}, \mathrm{CDCl}_{3}\right)$ :

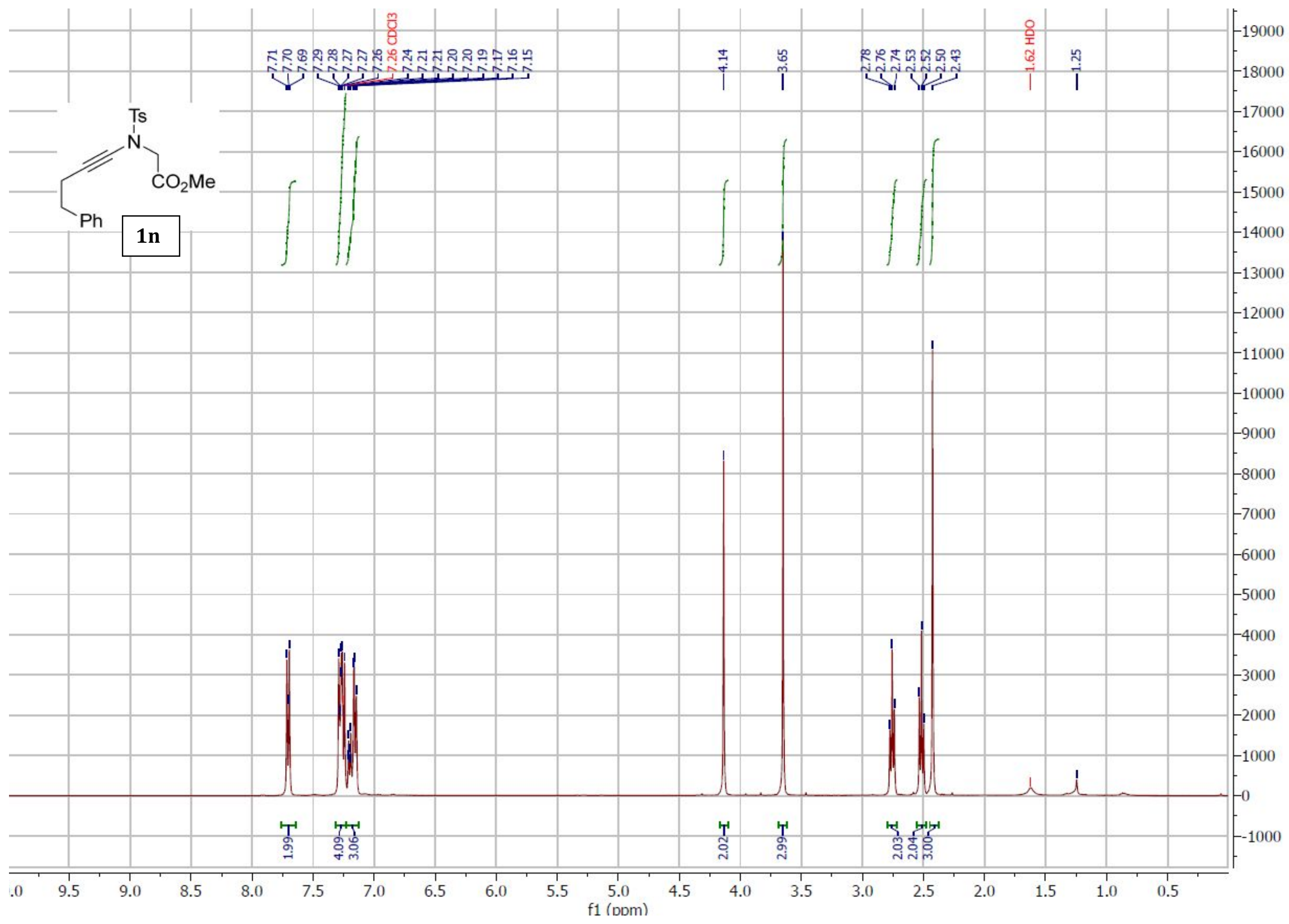


${ }^{13} \mathrm{C}$ NMR of compound $\mathbf{1 n}\left(101 \mathrm{MHz}, \mathrm{CDCl}_{3}\right)$ :

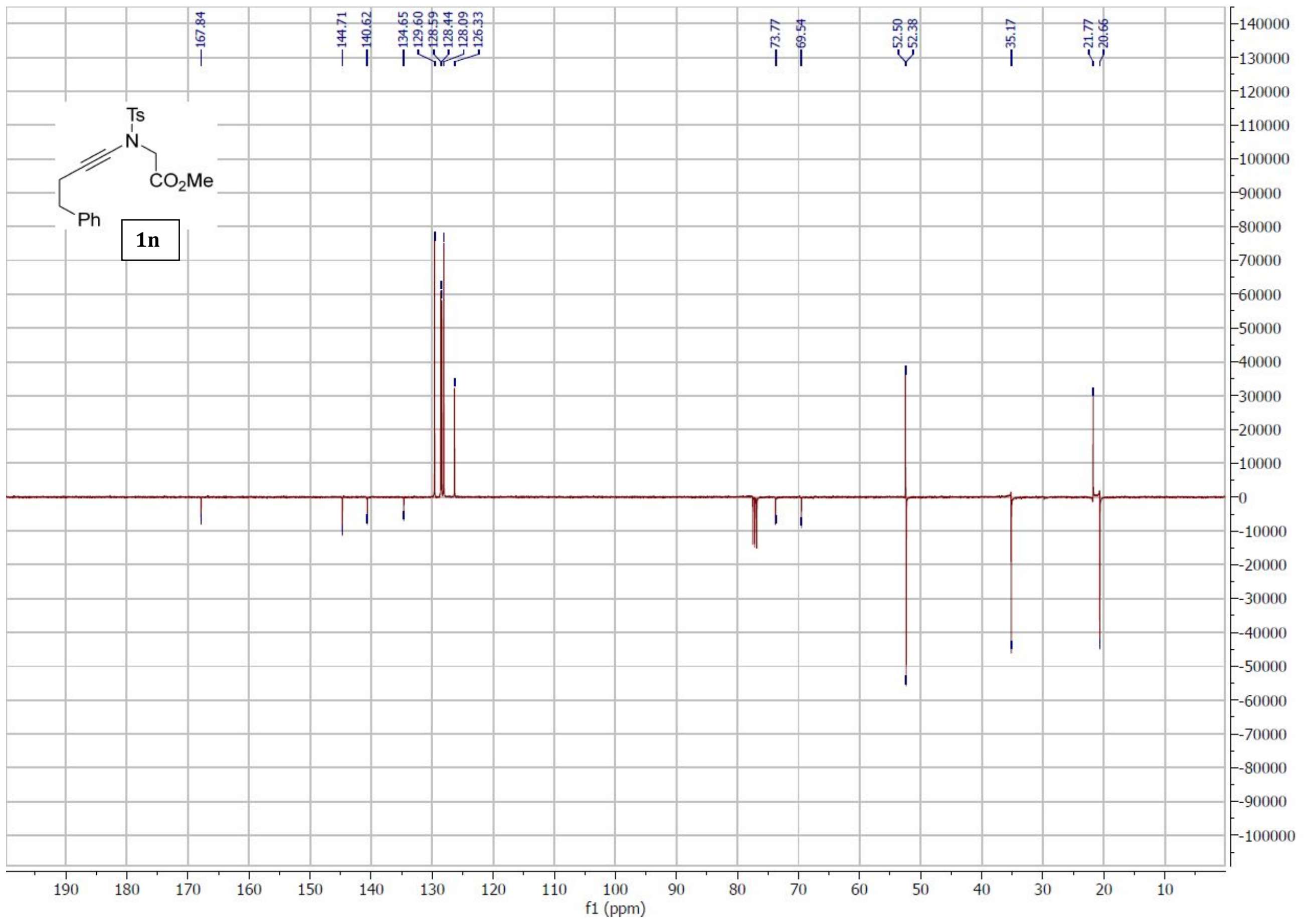


${ }^{1} \mathrm{H}$ NMR of compound $10\left(400 \mathrm{MHz}, \mathrm{CDCl}_{3}\right)$ :

RL-PH-AW-002-1-1905.1.fid

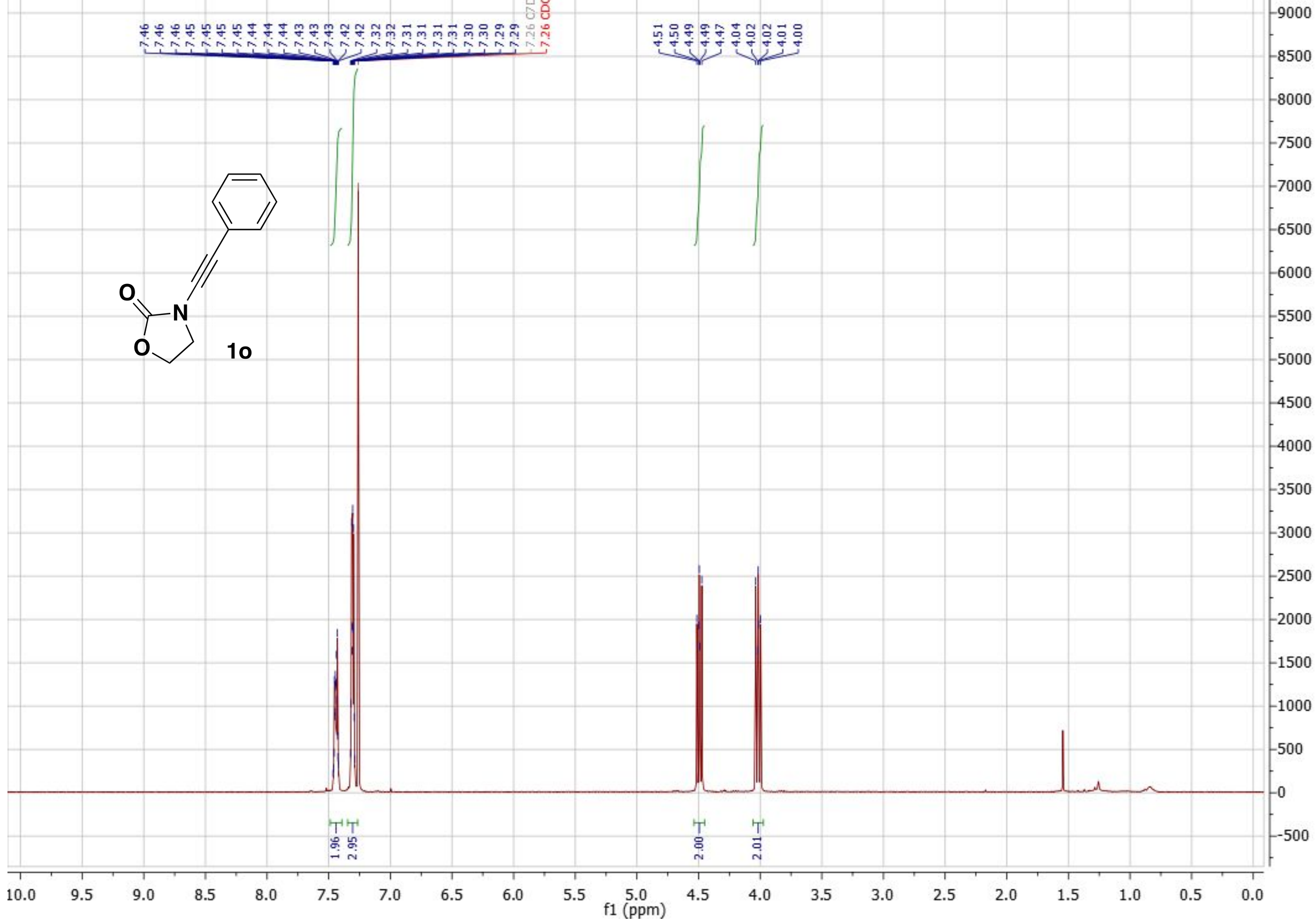


${ }^{1} \mathrm{H}$ NMR of compound $\mathbf{1 p}$ (400 $\mathrm{MHz}, \mathrm{CDCl}_{3}$ ):

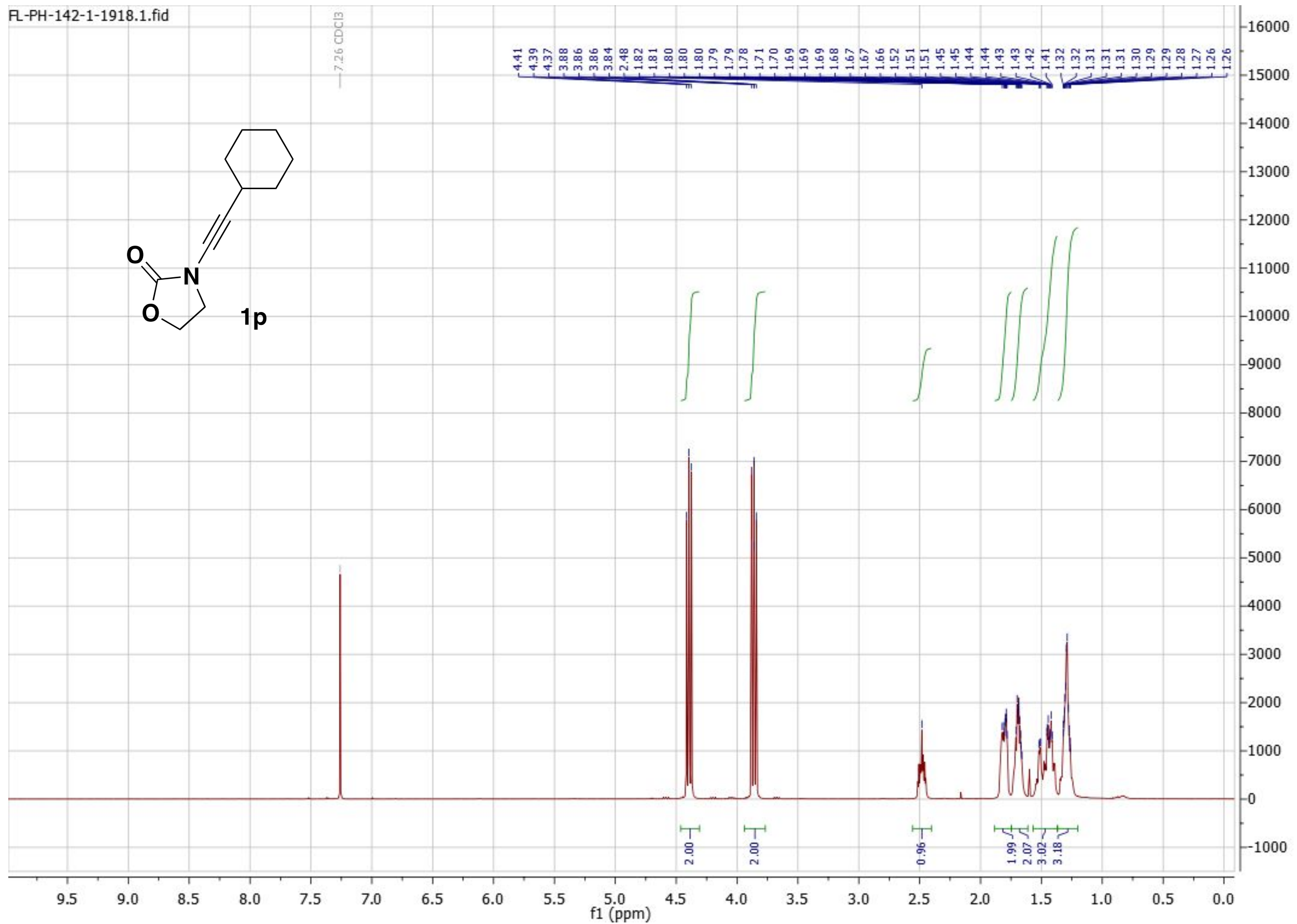


${ }^{1} \mathrm{H}$ NMR of compound 1q (400 MHz, $\left.\mathrm{CDCl}_{3}\right)$ :

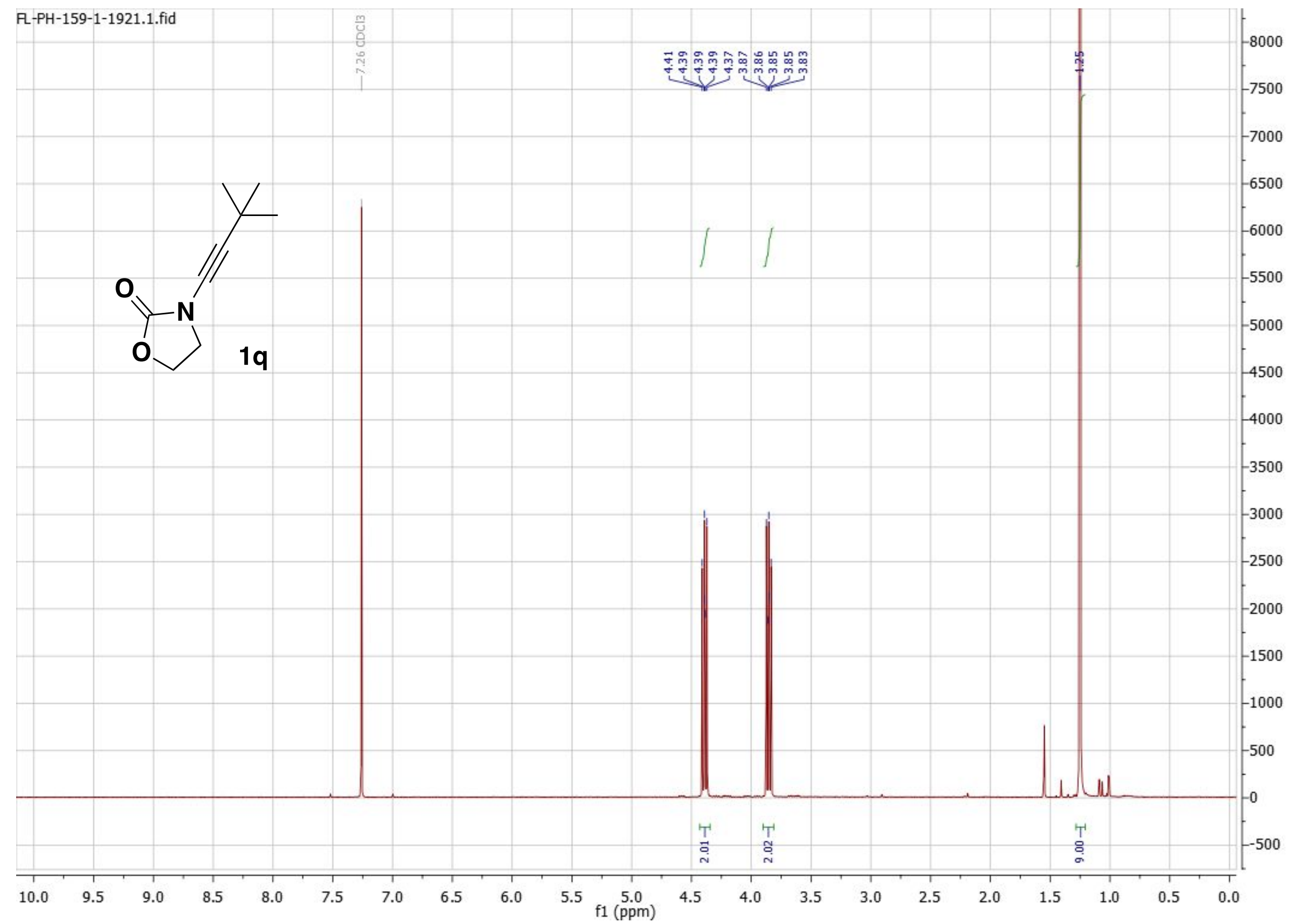


${ }^{1} \mathrm{H}$ NMR of compound $2 \mathbf{a}\left(400 \mathrm{MHz}, \mathrm{CDCl}_{3}\right)$ :

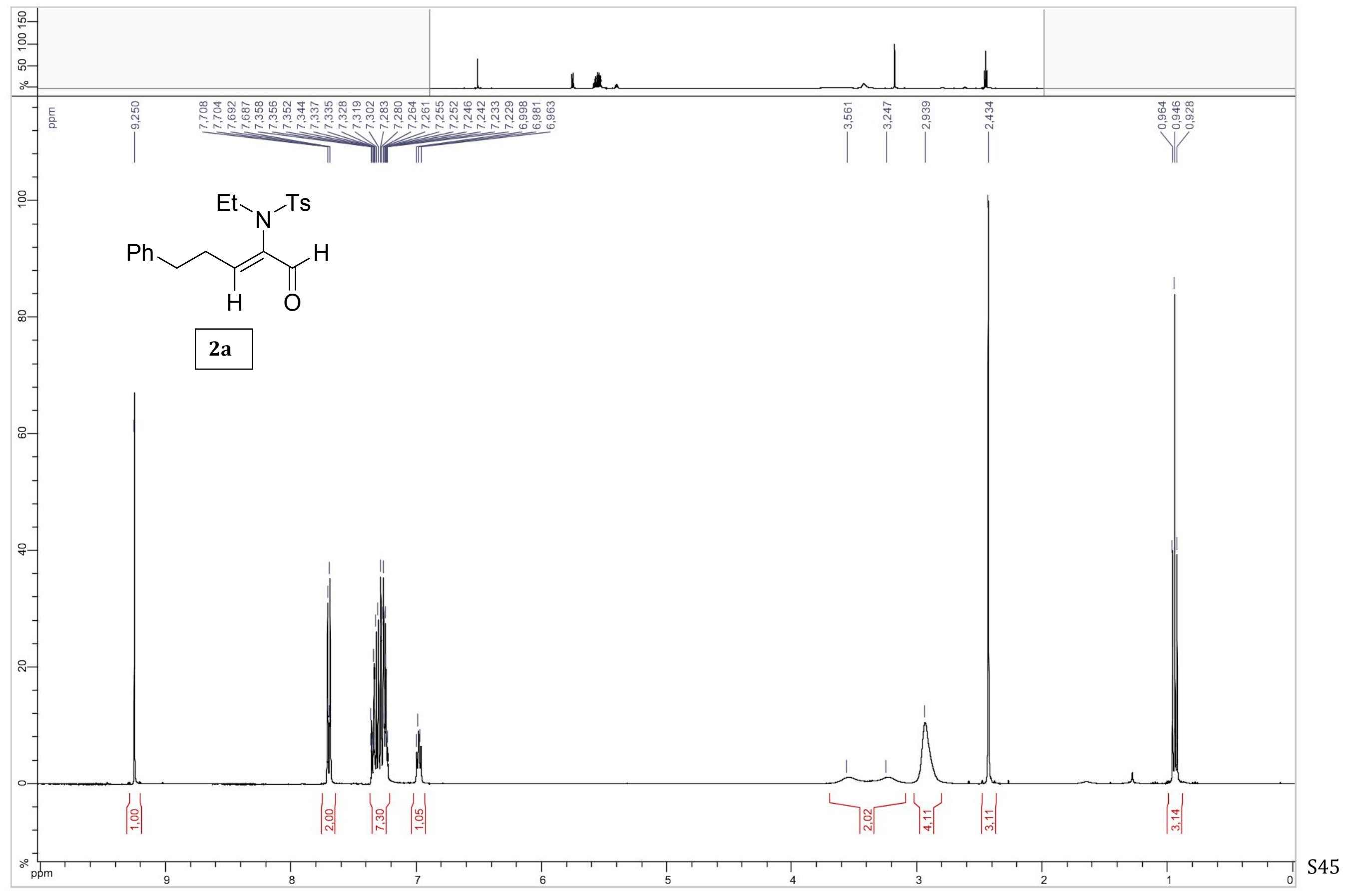


${ }^{13} \mathrm{C}$ NMR of compound $2 \mathbf{a}\left(101 \mathrm{MHz}, \mathrm{CDCl}_{3}\right)$ :

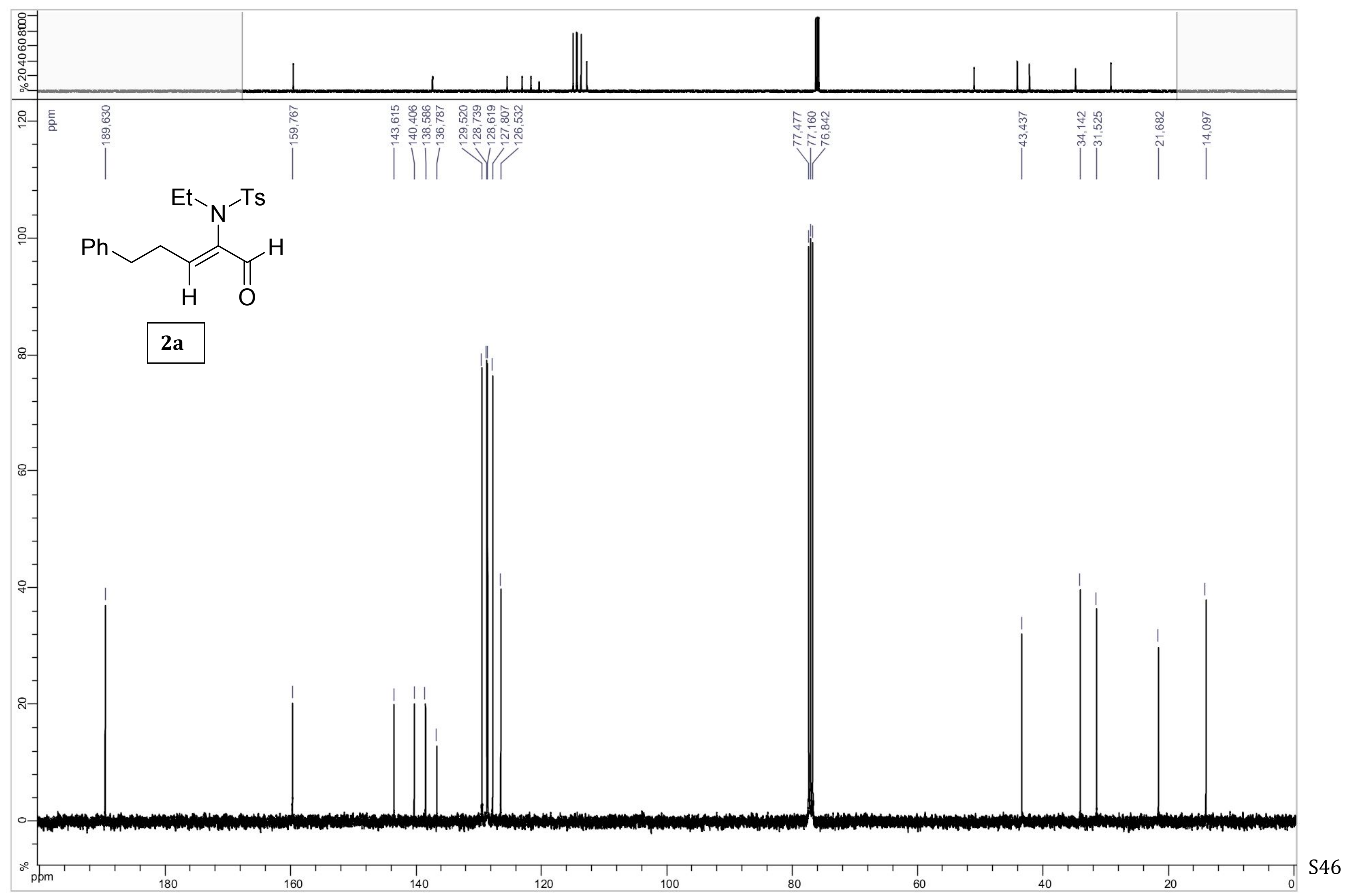


${ }^{1} \mathrm{H}$ NMR of compound 3a (400 MHz, $\mathrm{CDCl}_{3}$ ):

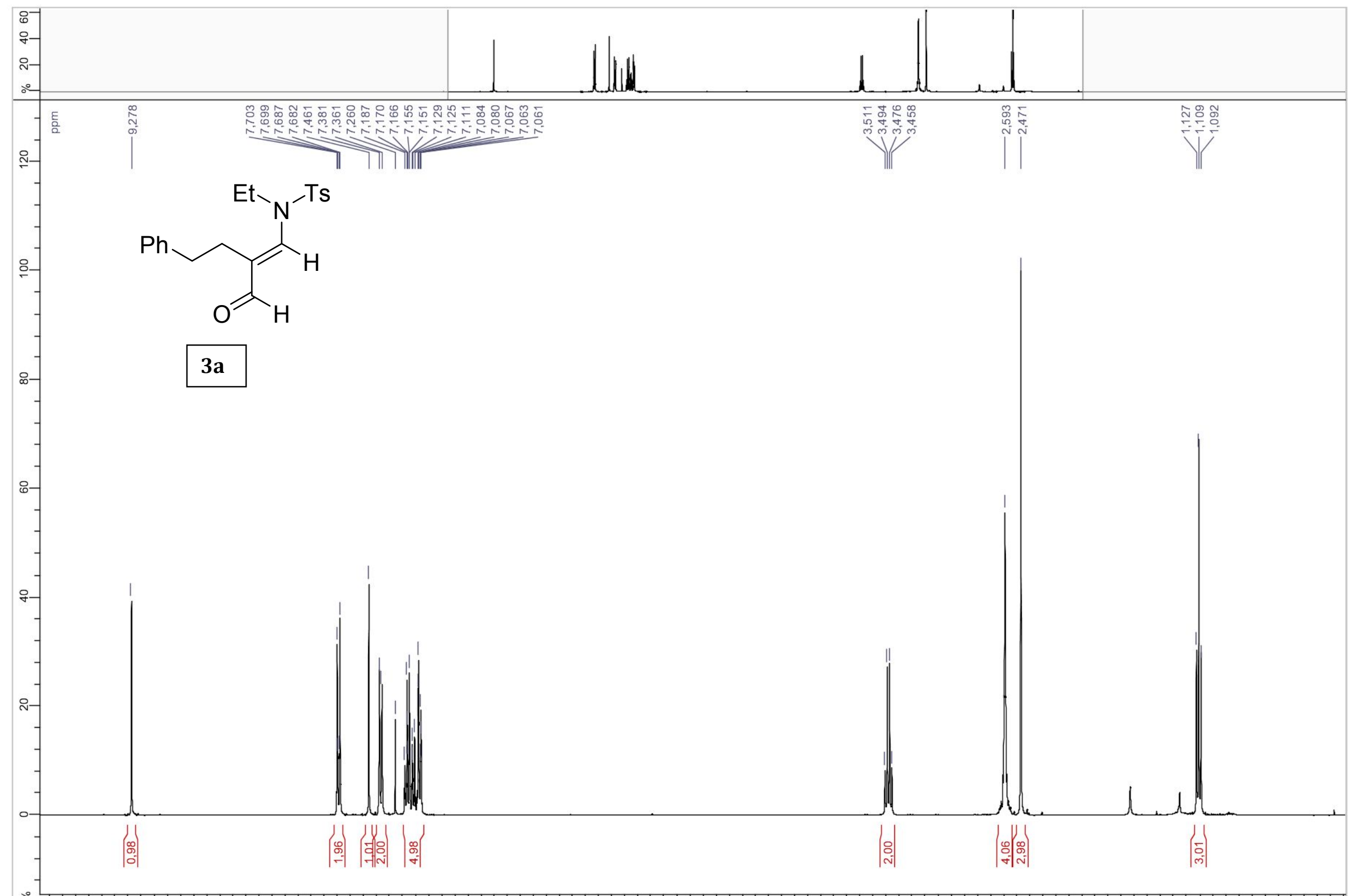


${ }^{13} \mathrm{C}$ NMR of compound 3a (101 MHz, $\mathrm{CDCl}_{3}$ ):

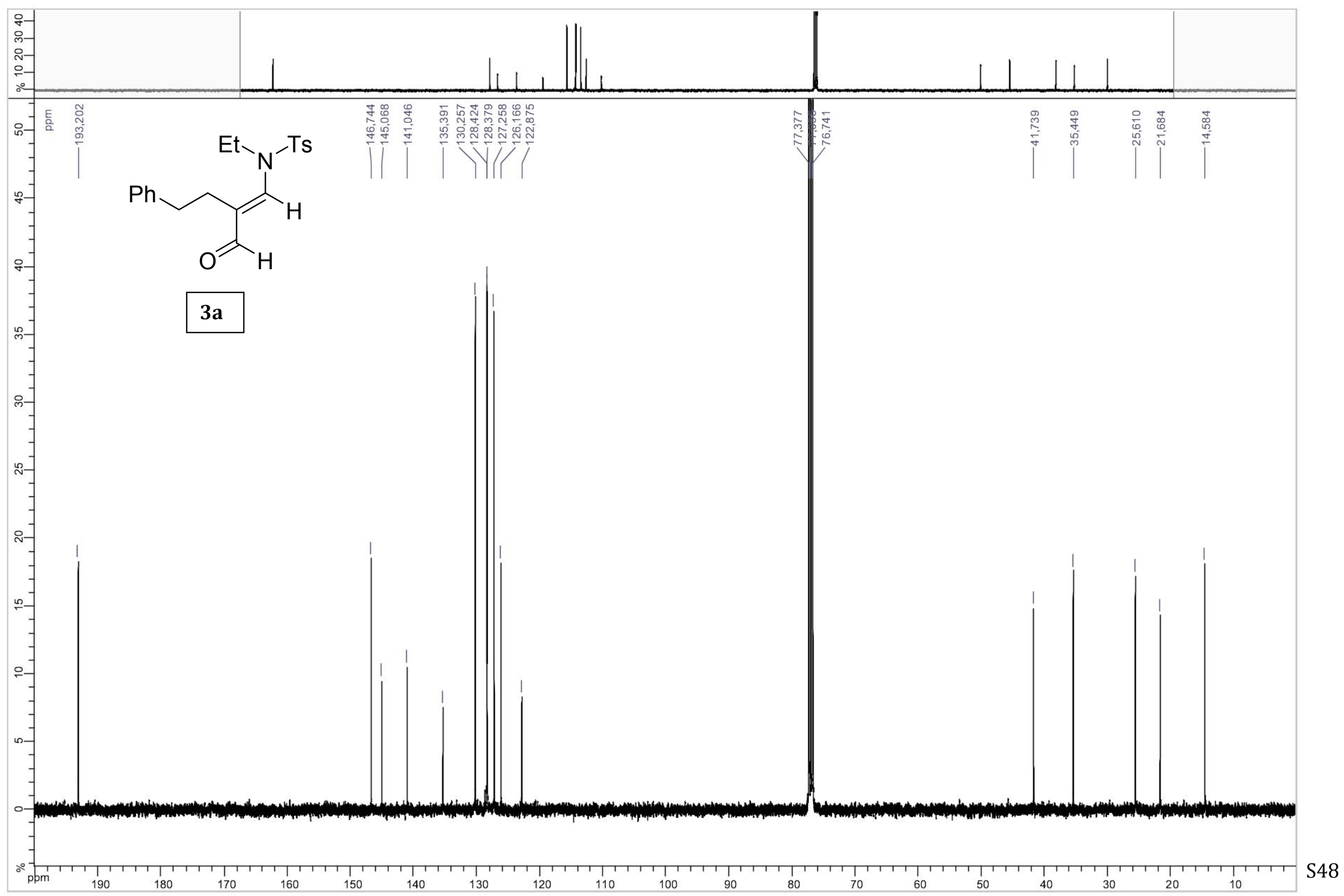


${ }^{1} \mathrm{H}$ NMR of compound $2 \mathbf{b}\left(400 \mathrm{MHz}, \mathrm{CDCl}_{3}\right)$ :

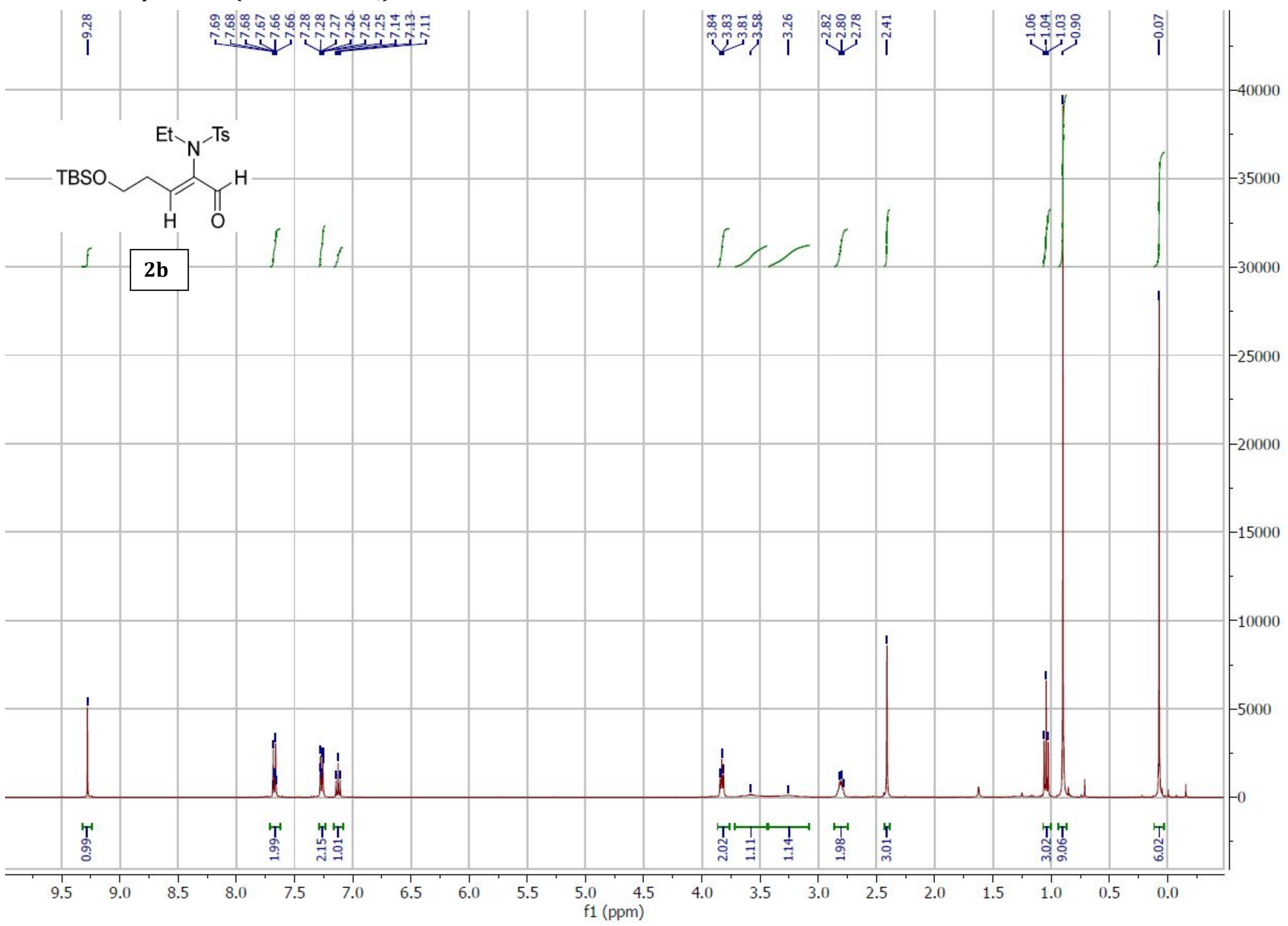


${ }^{13} \mathrm{C}$ NMR of compound $\mathbf{2 b}\left(101 \mathrm{MHz}, \mathrm{CDCl}_{3}\right)$ :

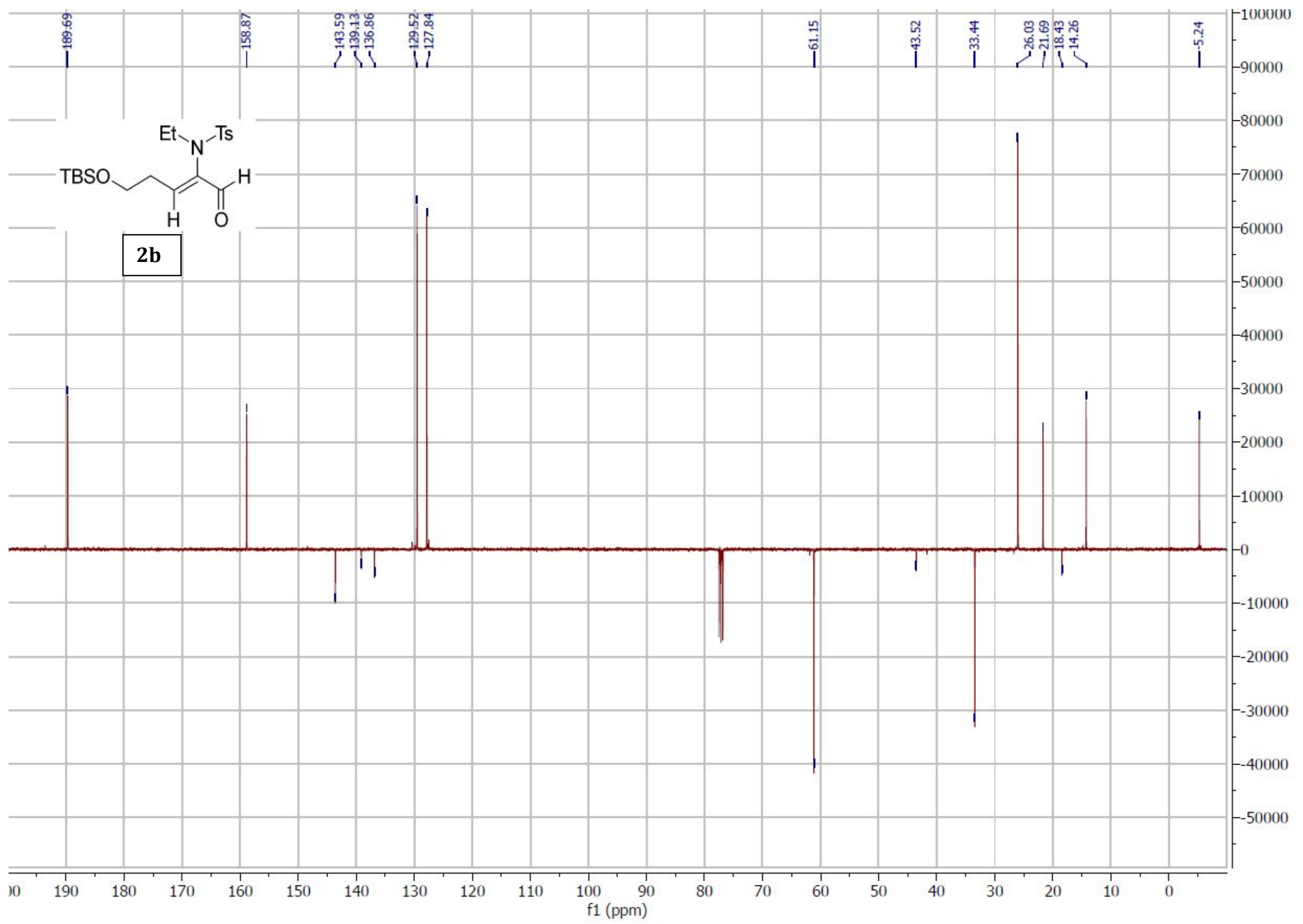


${ }^{1} \mathrm{H}$ NMR of compound $\mathbf{3 b}\left(500 \mathrm{MHz}, \mathrm{CDCl}_{3}\right)$ :

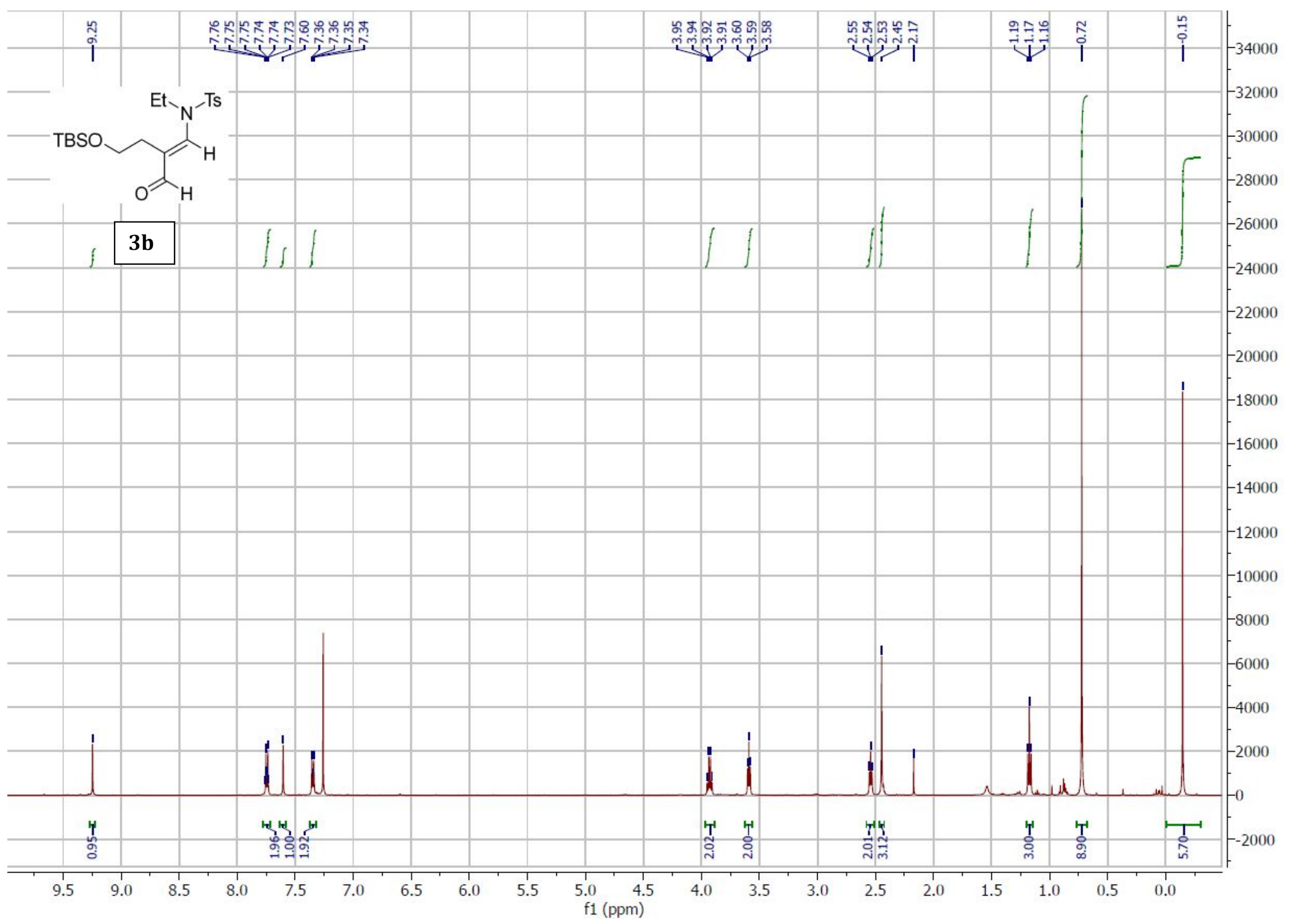


${ }^{13} \mathrm{C}$ NMR of compound $\mathbf{3 b}\left(126 \mathrm{MHz}, \mathrm{CDCl}_{3}\right)$ :

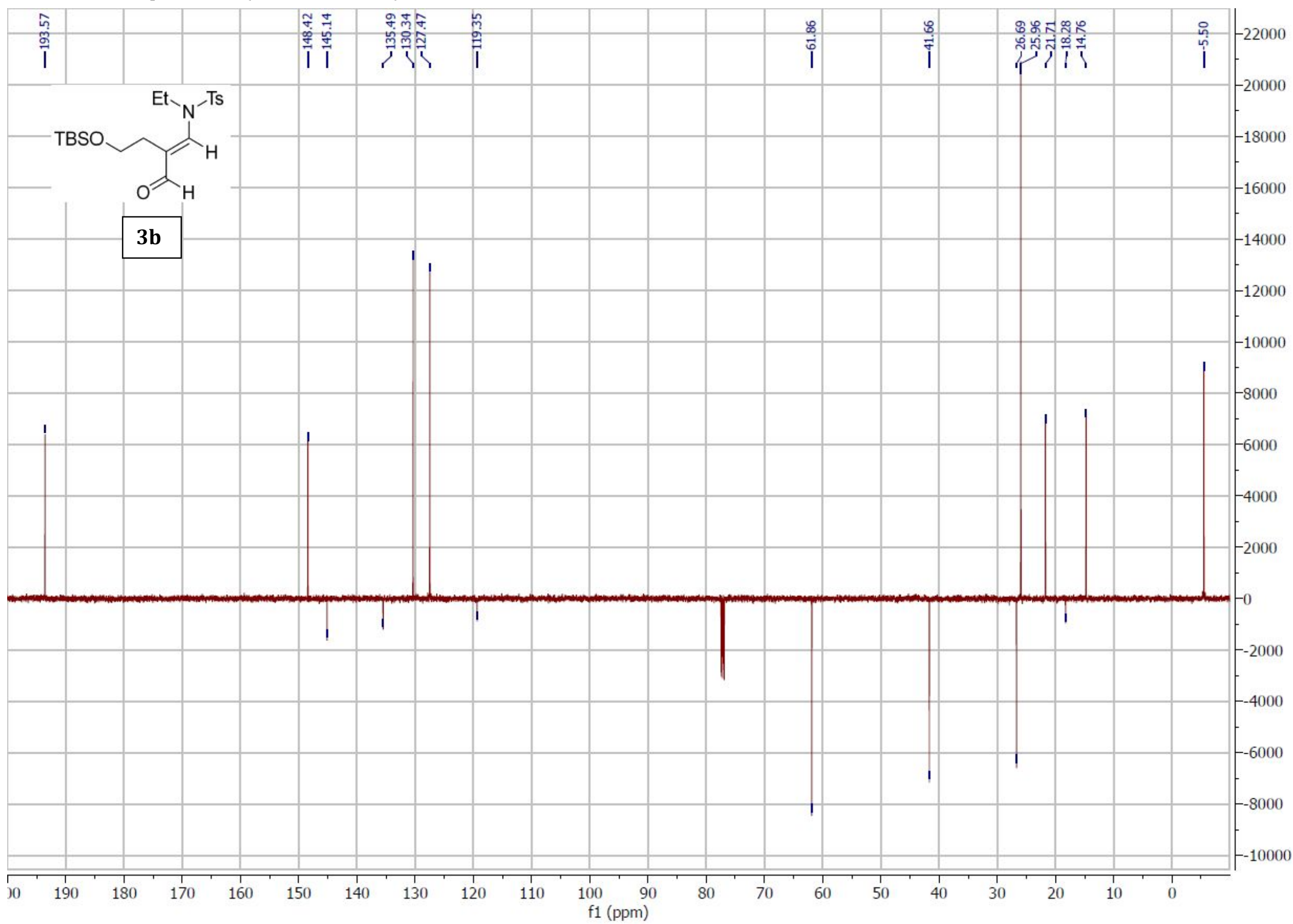


${ }^{1} \mathrm{H}$ NMR of compound 2c (400 MHz, $\left.\mathrm{CDCl}_{3}\right)$ :

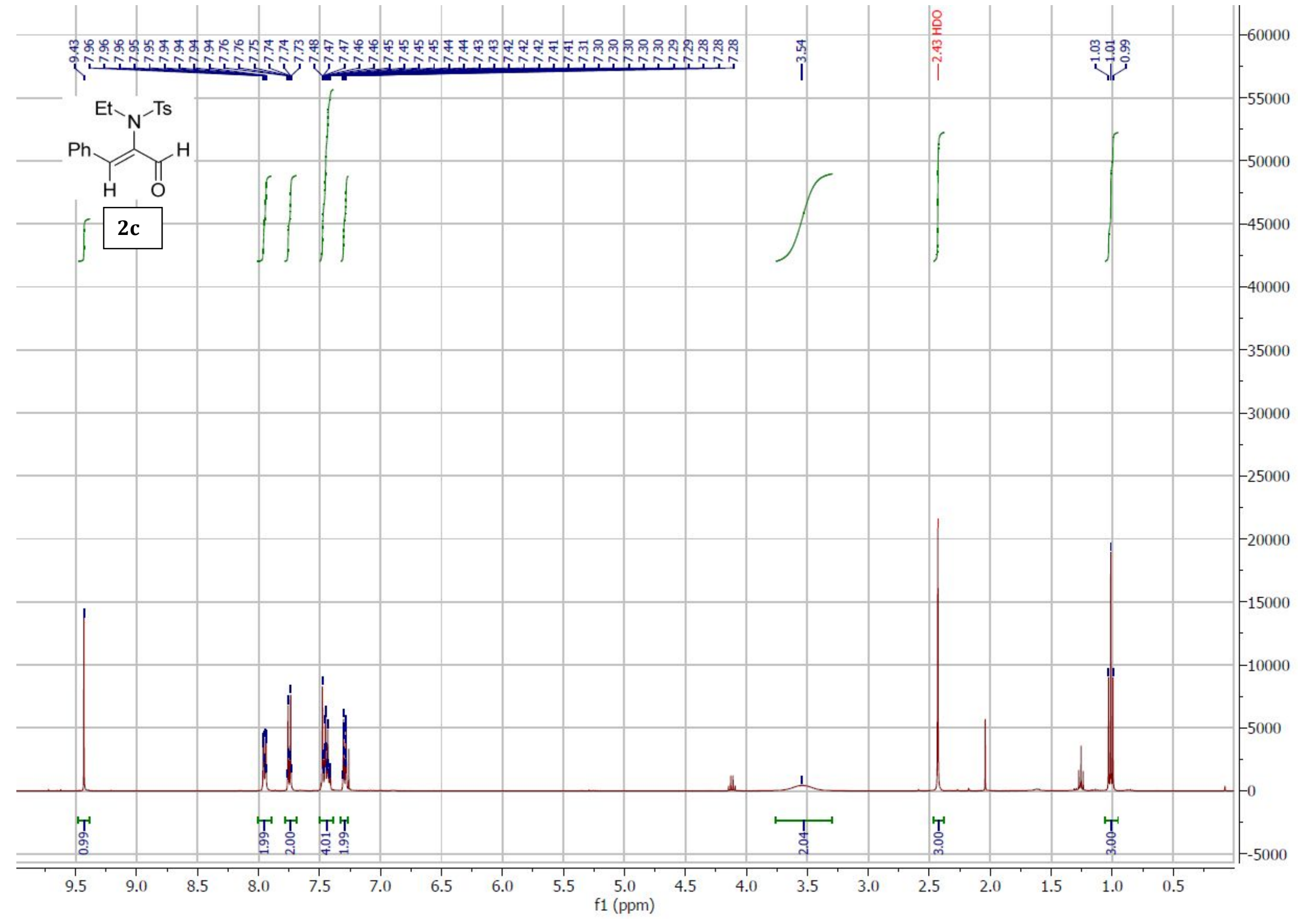


${ }^{13} \mathrm{C}$ NMR of compound 2c (101 MHz, $\left.\mathrm{CDCl}_{3}\right)$ :

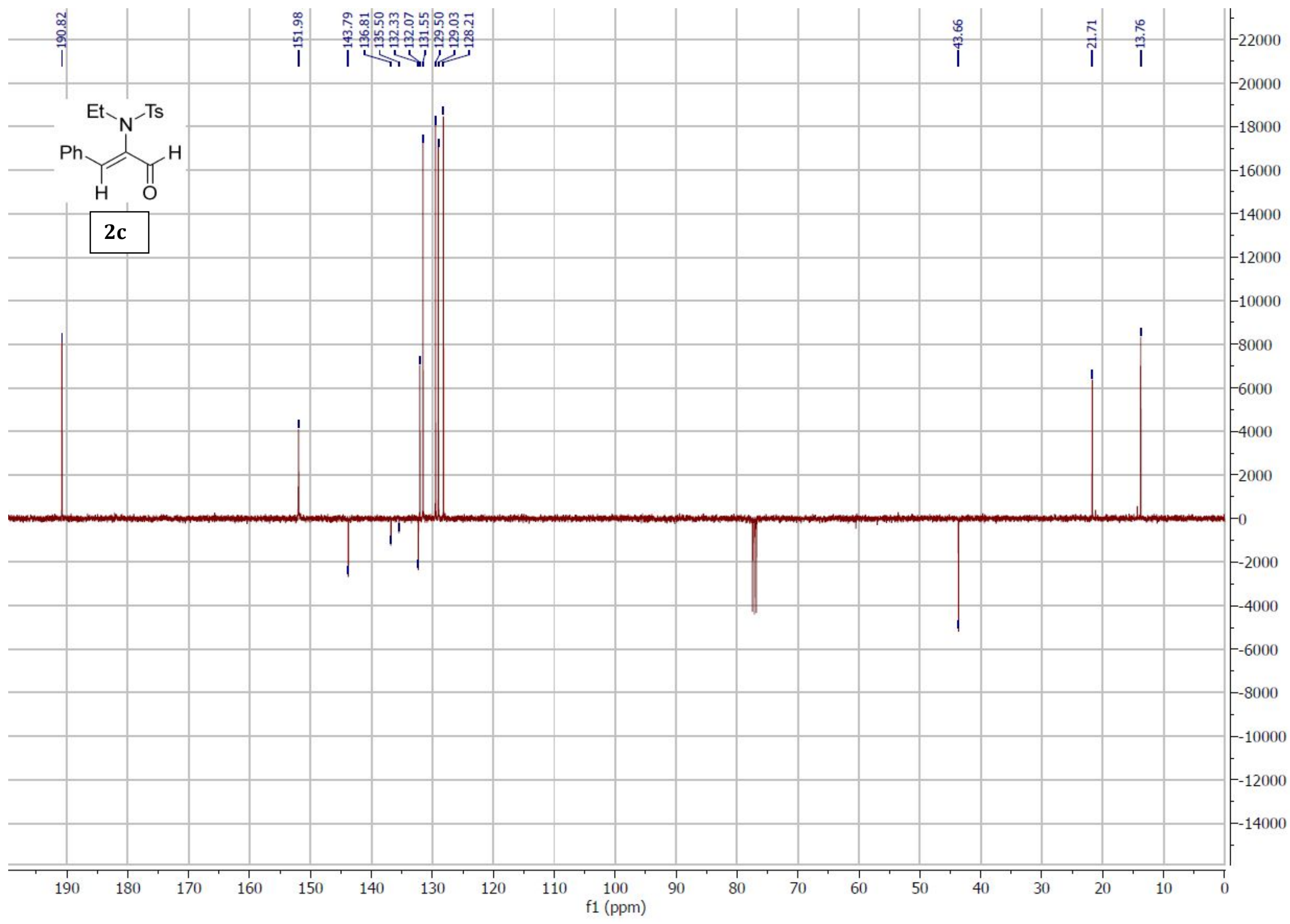


${ }^{1} \mathrm{H}$ NMR of compound $3 c\left(400 \mathrm{MHz}, \mathrm{CDCl}_{3}\right)$ :

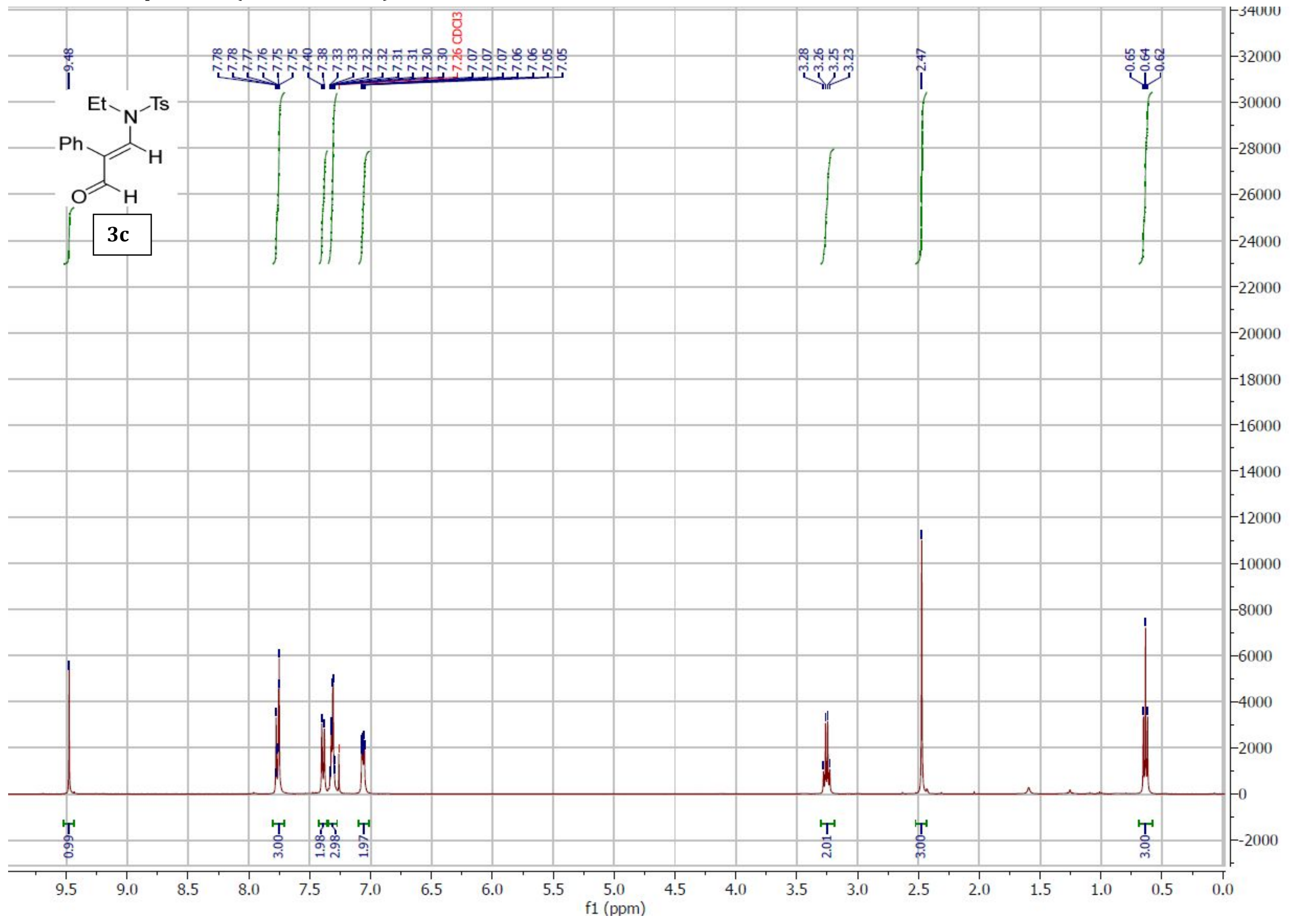


${ }^{13} \mathrm{C}$ NMR of compound 3c (101 MHz, $\left.\mathrm{CDCl}_{3}\right)$ :

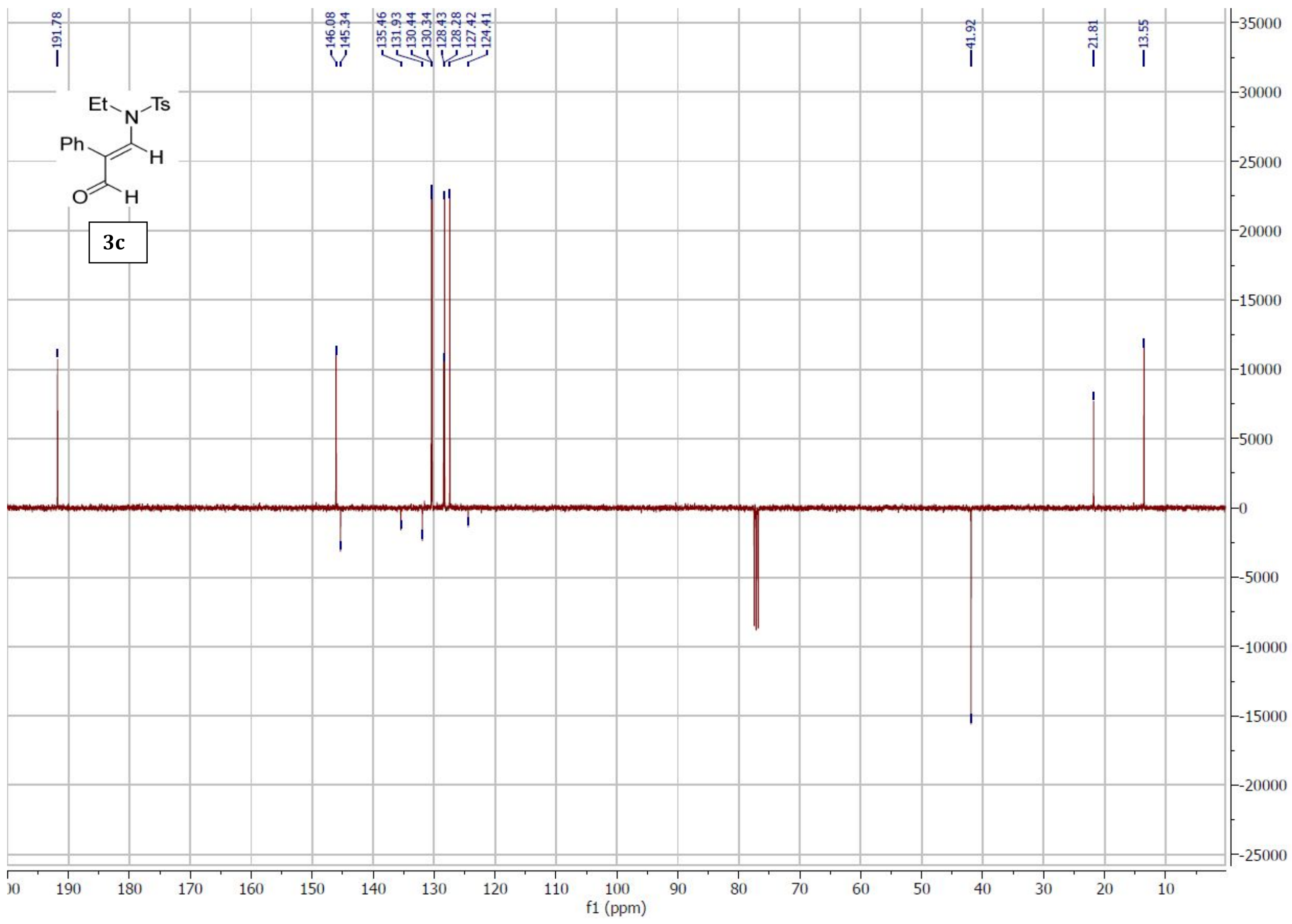


${ }^{1} \mathrm{H}$ NMR of compound $3 \mathbf{e}\left(400 \mathrm{MHz}, \mathrm{CDCl}_{3}\right)$ :

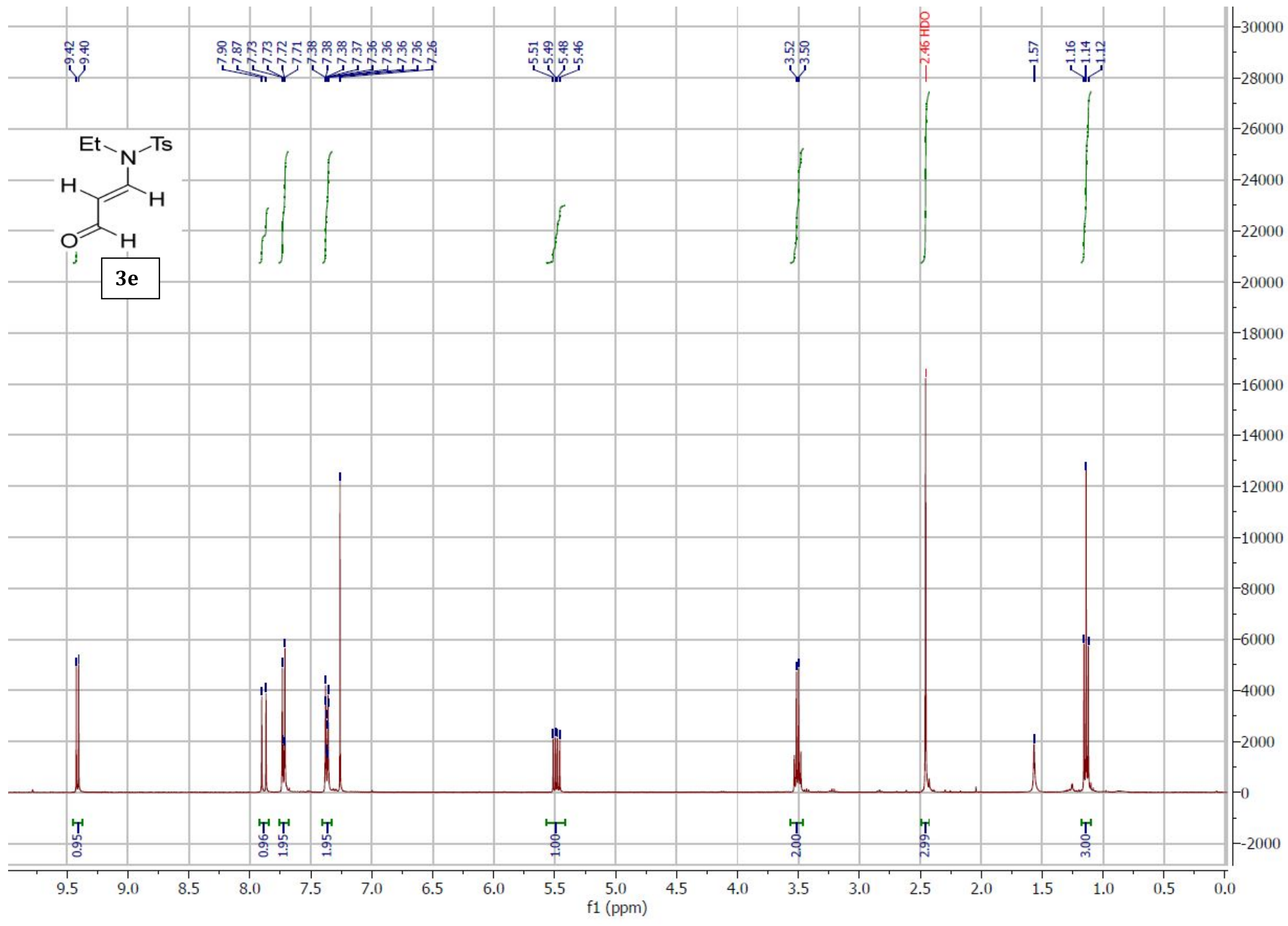


${ }^{13} \mathrm{C}$ NMR of compound $\mathbf{3 e}\left(101 \mathrm{MHz}, \mathrm{CDCl}_{3}\right)$ :

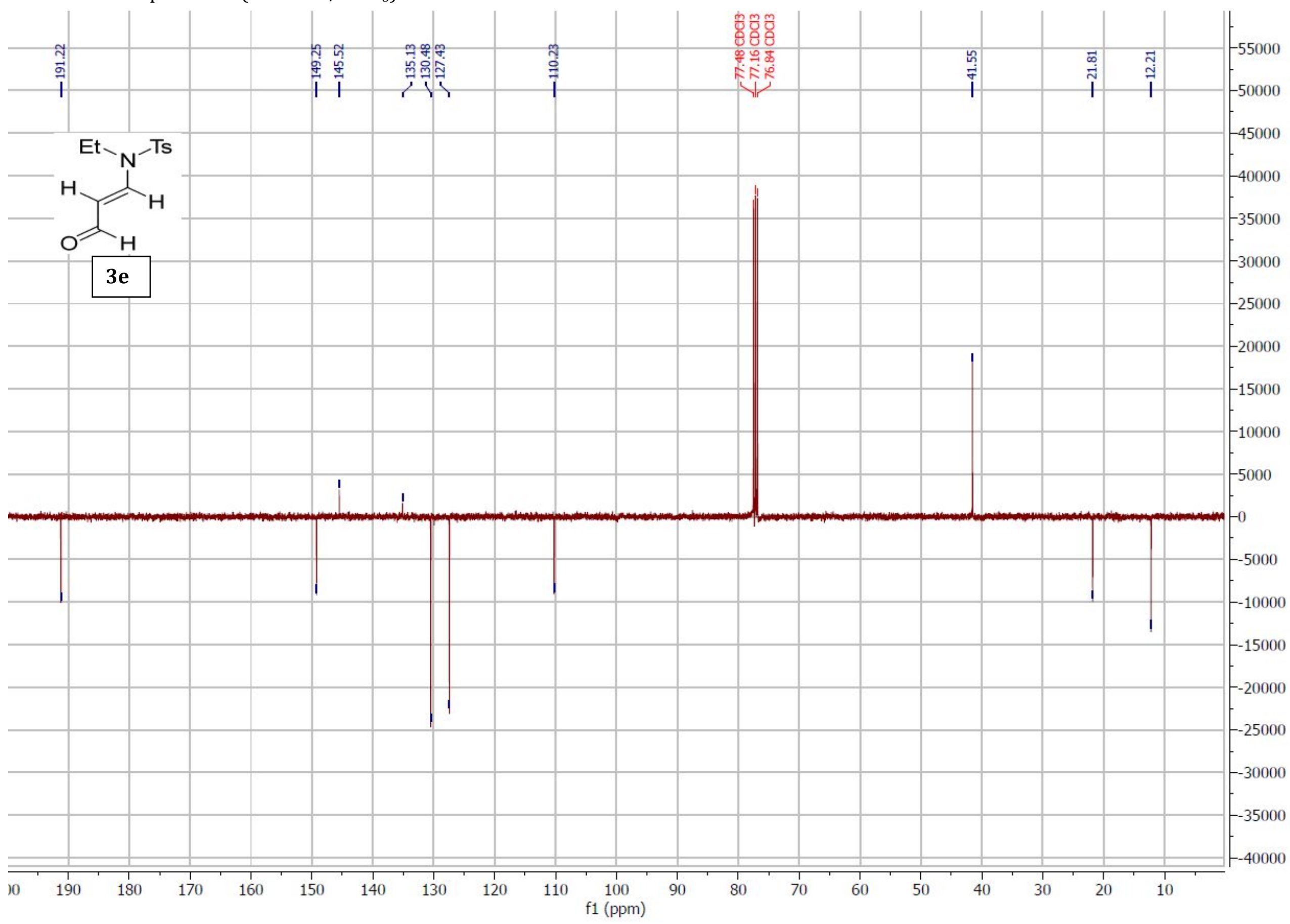


${ }^{1} \mathrm{H}$ NMR of compound $2 \mathbf{f}\left(400 \mathrm{MHz}, \mathrm{CDCl}_{3}\right)$ :

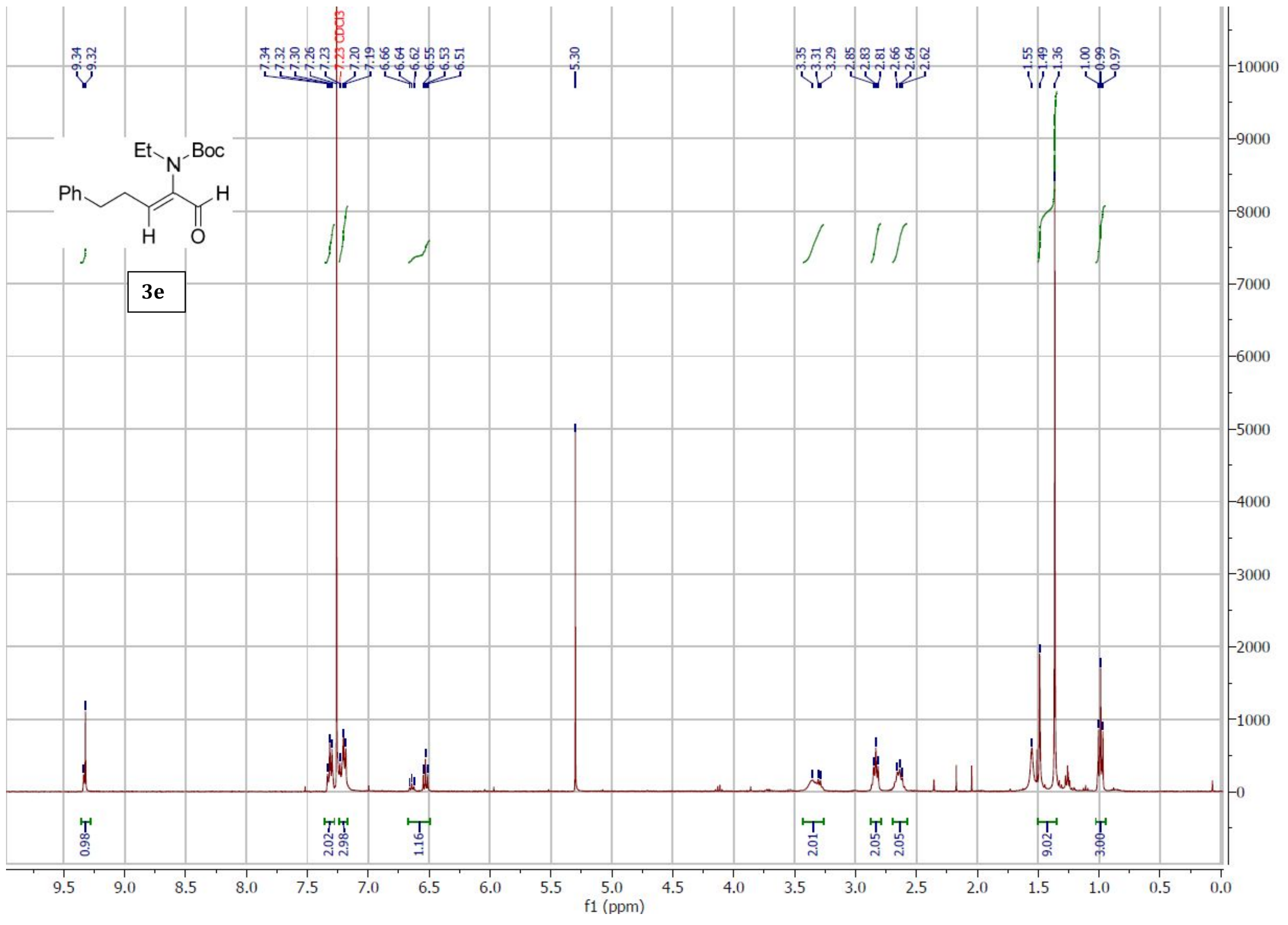


${ }^{13} \mathrm{C}$ NMR of compound $2 \mathbf{f}\left(101 \mathrm{MHz}, \mathrm{CDCl}_{3}\right)$ :

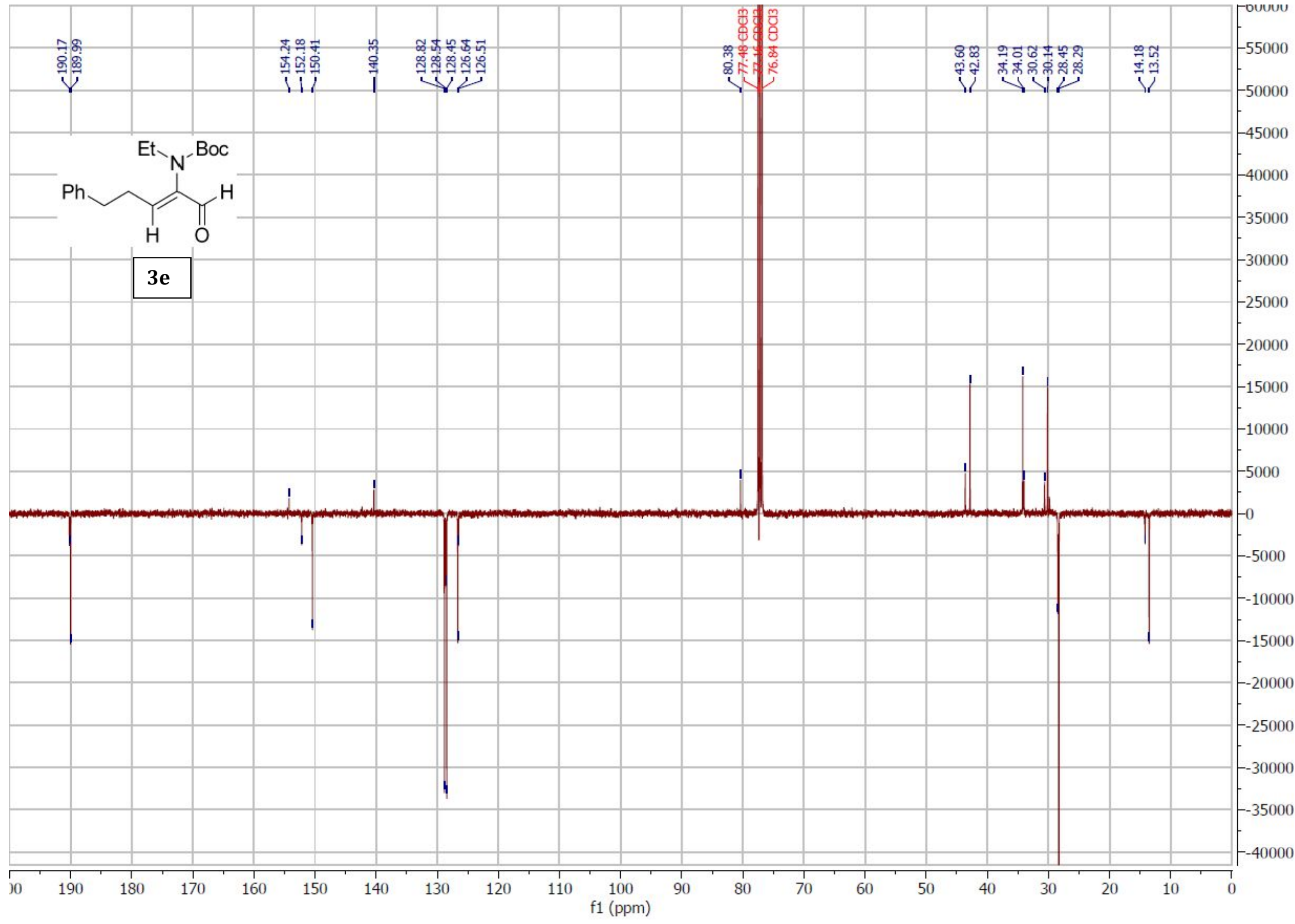


${ }^{1} \mathrm{H}$ NMR of compound $3 f\left(400 \mathrm{MHz}, \mathrm{CDCl}_{3}\right)$ :

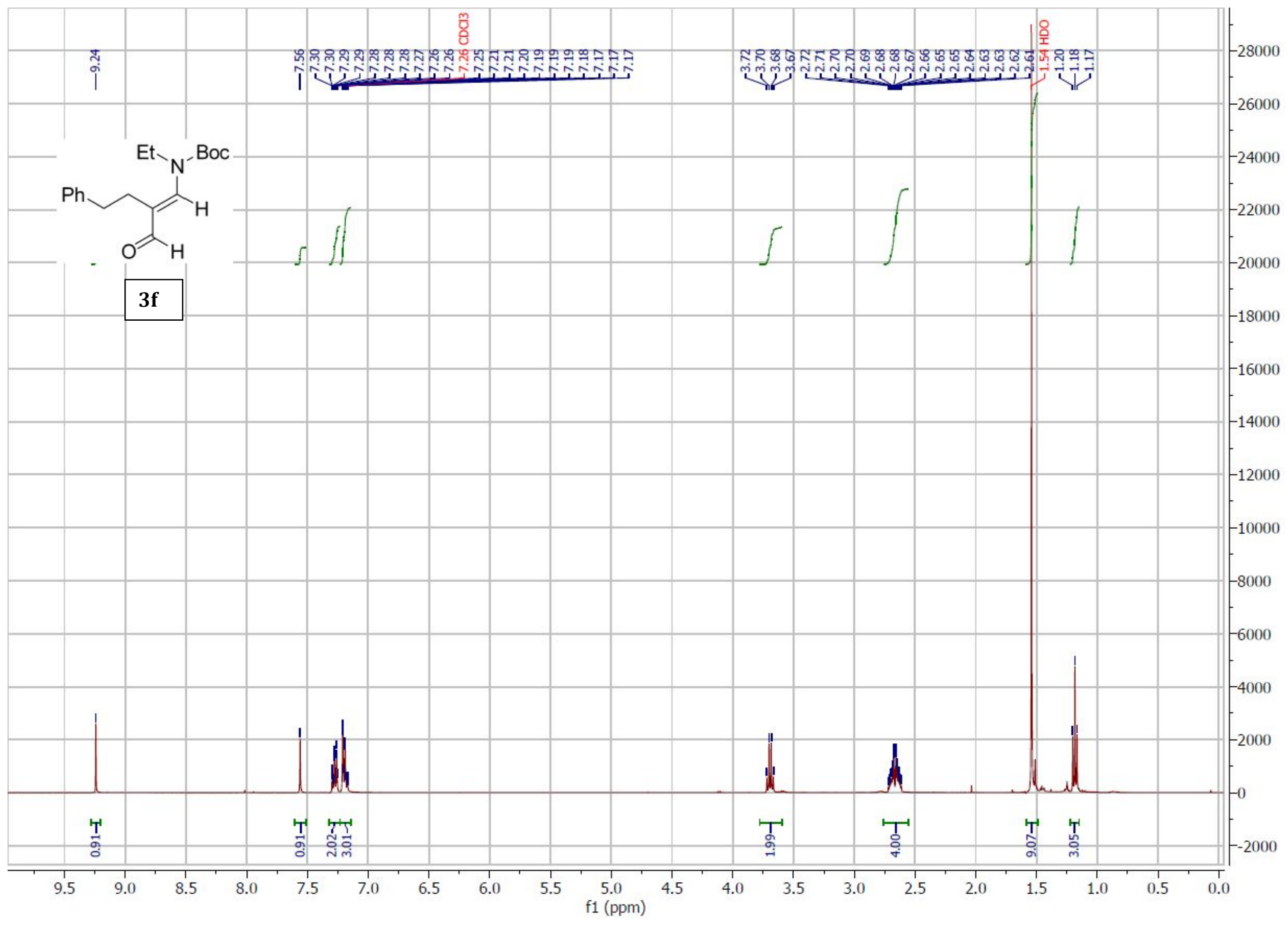


${ }^{13} \mathrm{C}$ NMR of compound $\mathbf{3 f}\left(101 \mathrm{MHz}, \mathrm{CDCl}_{3}\right)$ :

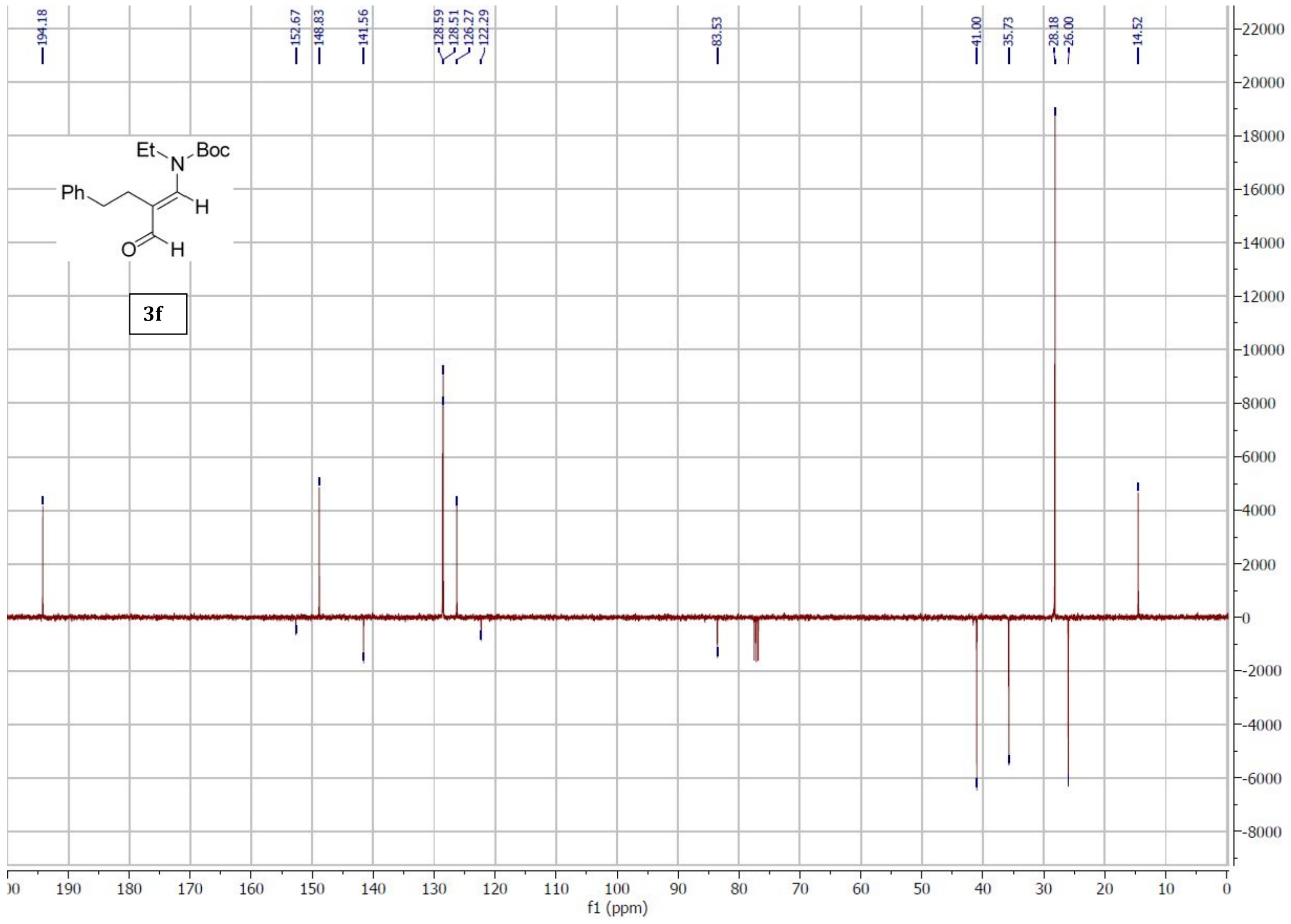


${ }^{1} \mathrm{H}$ NMR of compound $2 \mathrm{~g}$ (400 MHz, $\mathrm{CDCl}_{3}$ ):

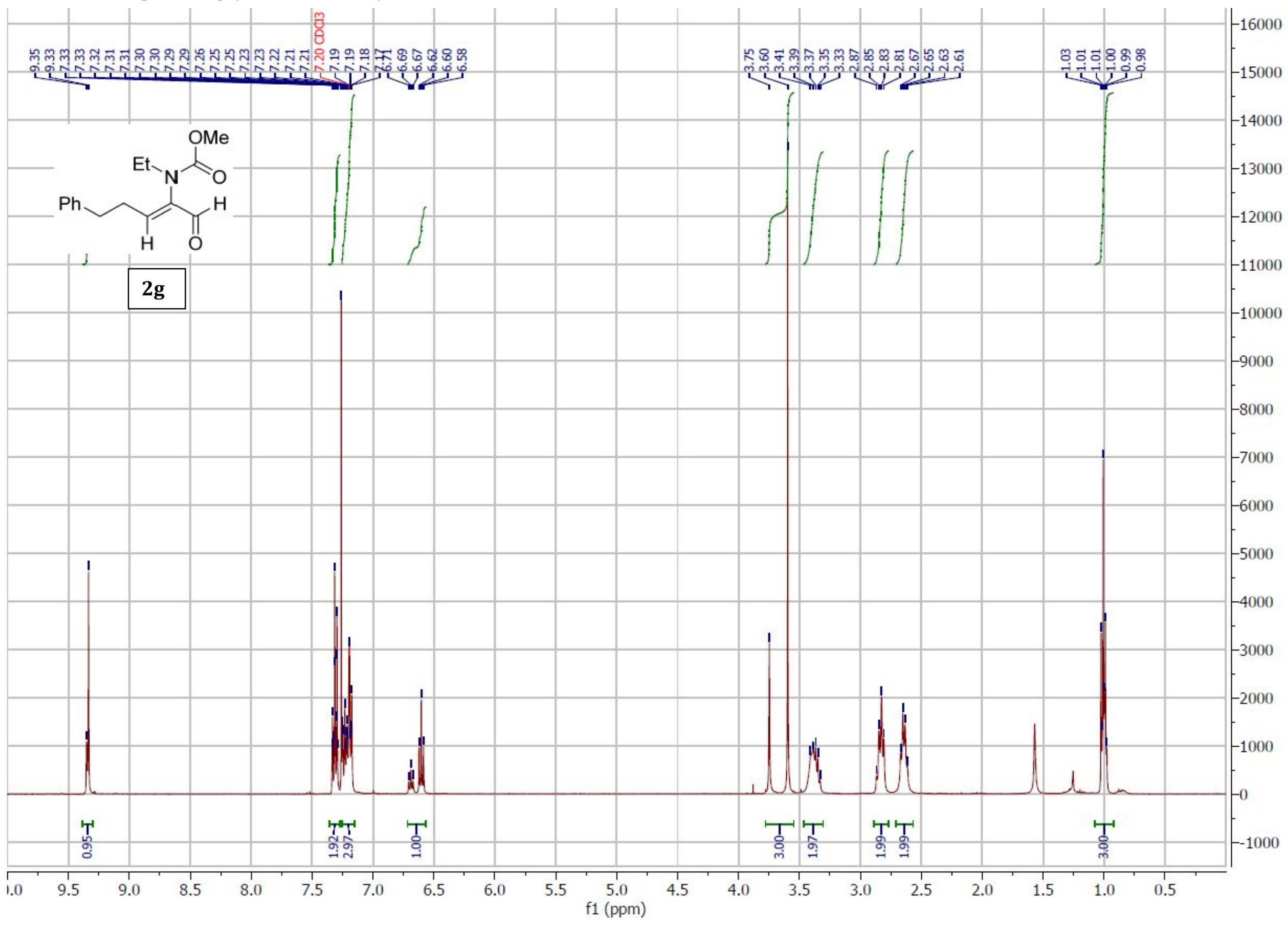


${ }^{13} \mathrm{C}$ NMR of compound $2 \mathbf{g}\left(101 \mathrm{MHz}, \mathrm{CDCl}_{3}\right)$ :

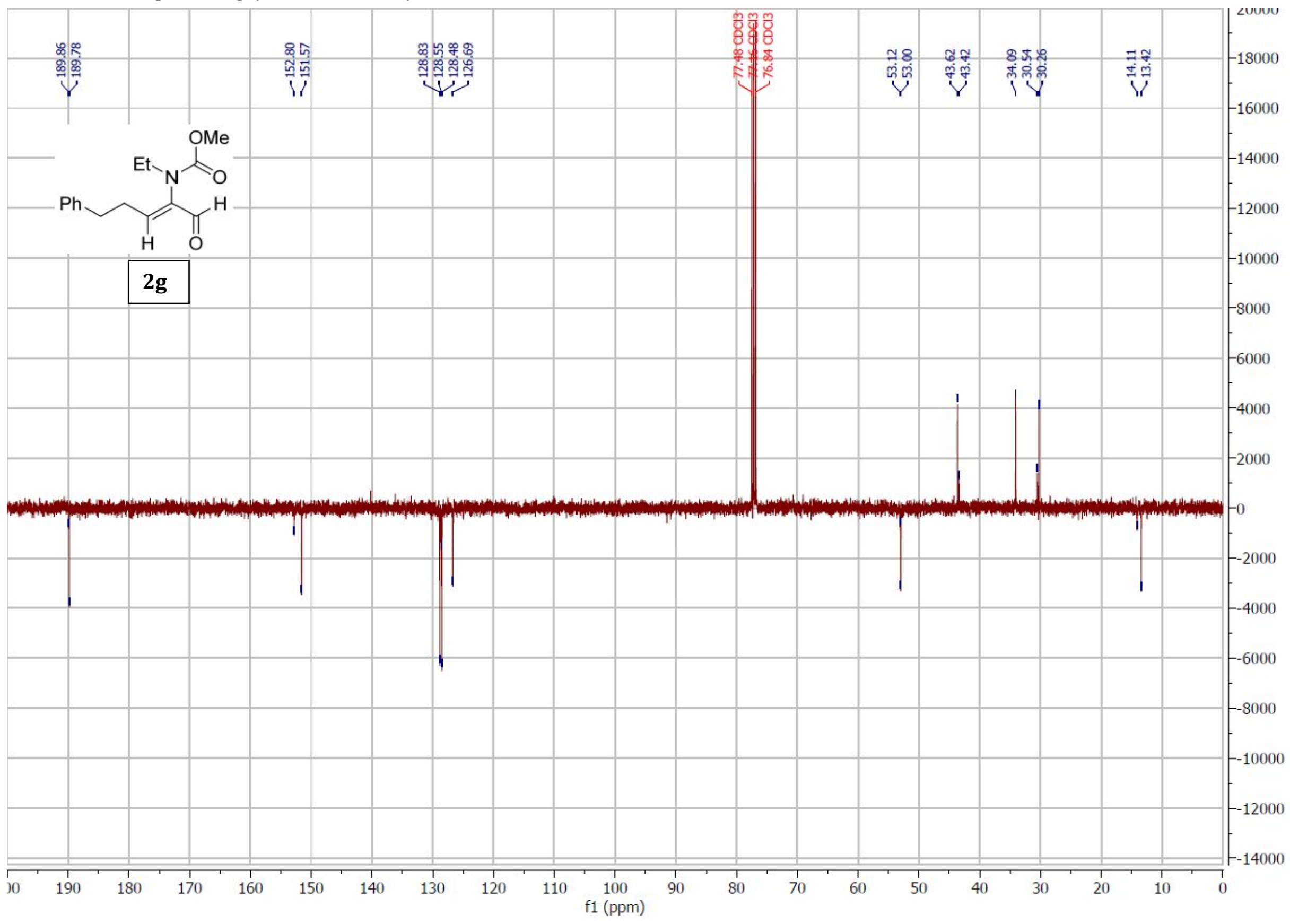


${ }^{1} \mathrm{H}$ NMR of compound $3 \mathbf{g}$ (400 MHz, $\mathrm{CDCl}_{3}$ ):

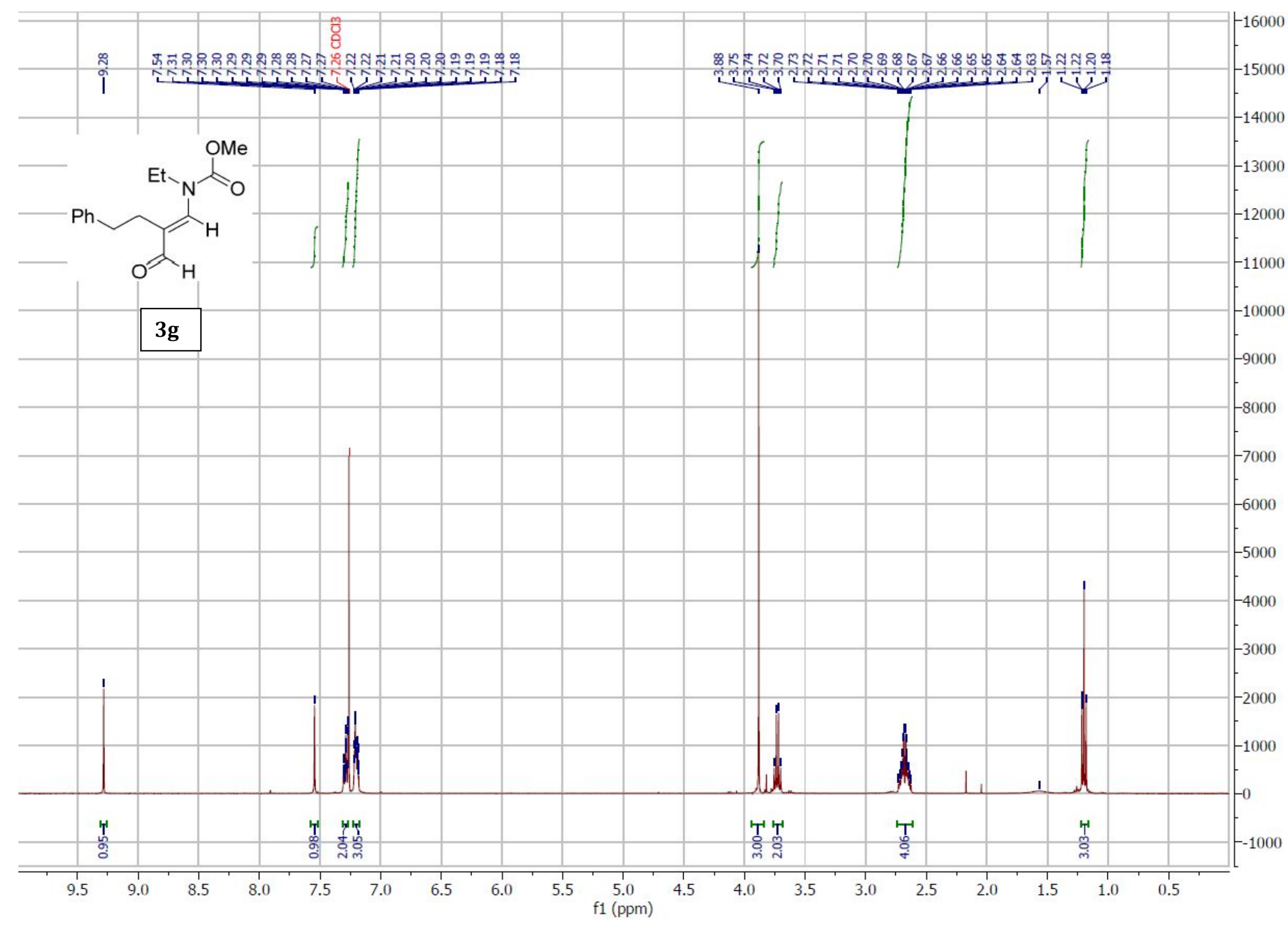


${ }^{13} \mathrm{C}$ NMR of compound $\mathbf{3 g}\left(101 \mathrm{MHz}, \mathrm{CDCl}_{3}\right)$ :

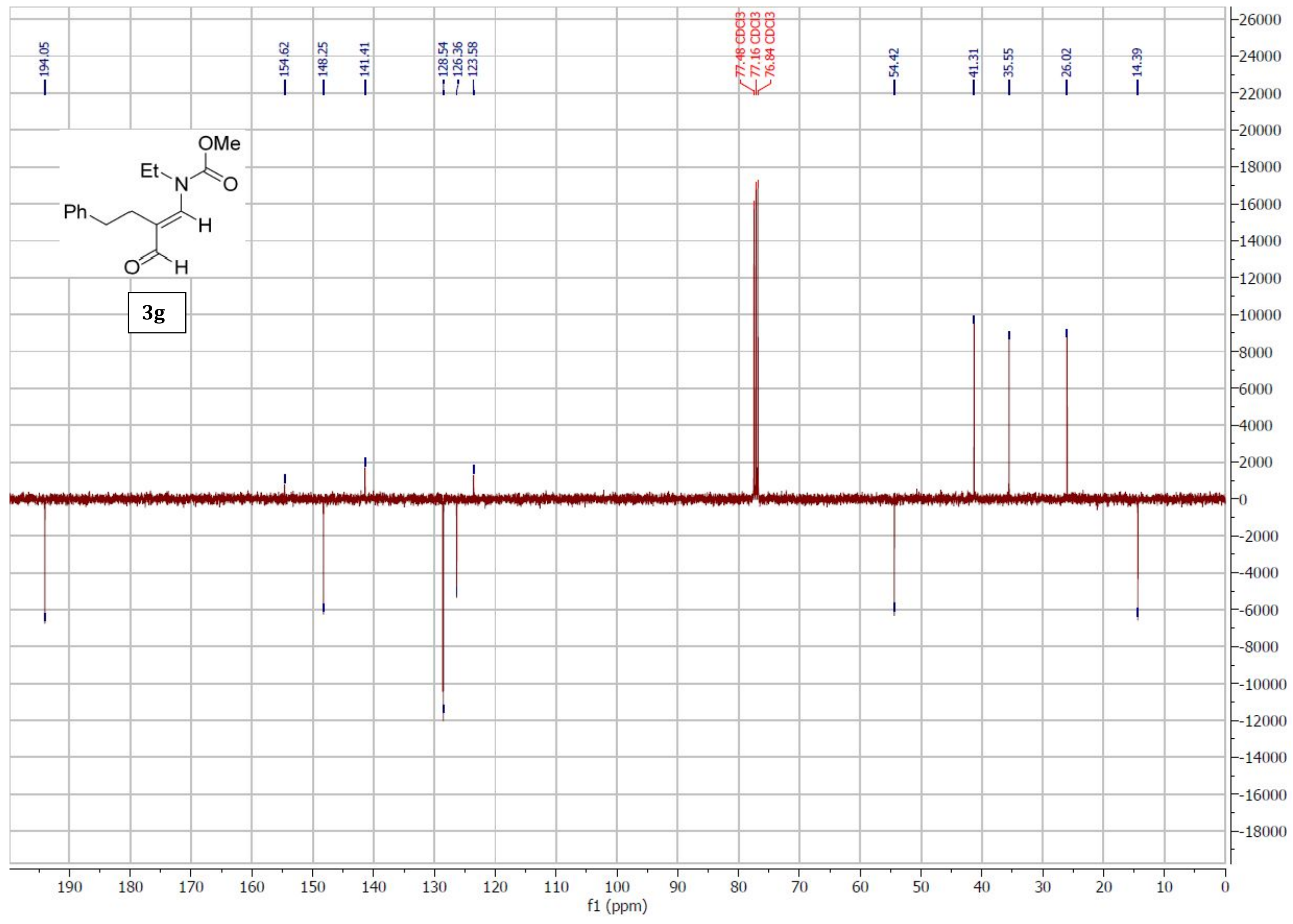


${ }^{1} \mathrm{H}$ NMR of compound $\mathbf{2 h}\left(400 \mathrm{MHz}, \mathrm{CDCl}_{3}\right)$ :

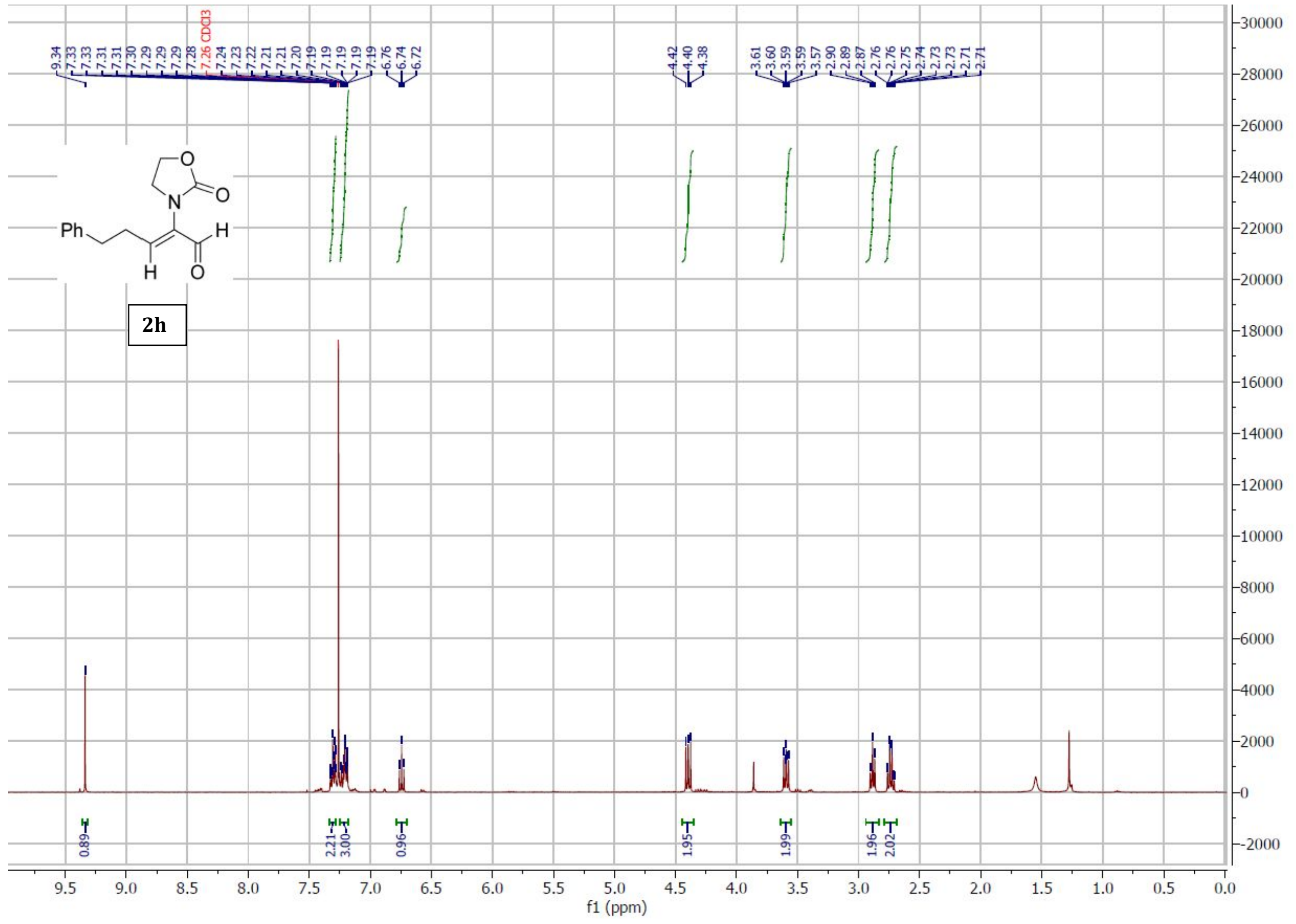


${ }^{13} \mathrm{C}$ NMR of compound $\mathbf{2 h}\left(101 \mathrm{MHz}, \mathrm{CDCl}_{3}\right)$ :

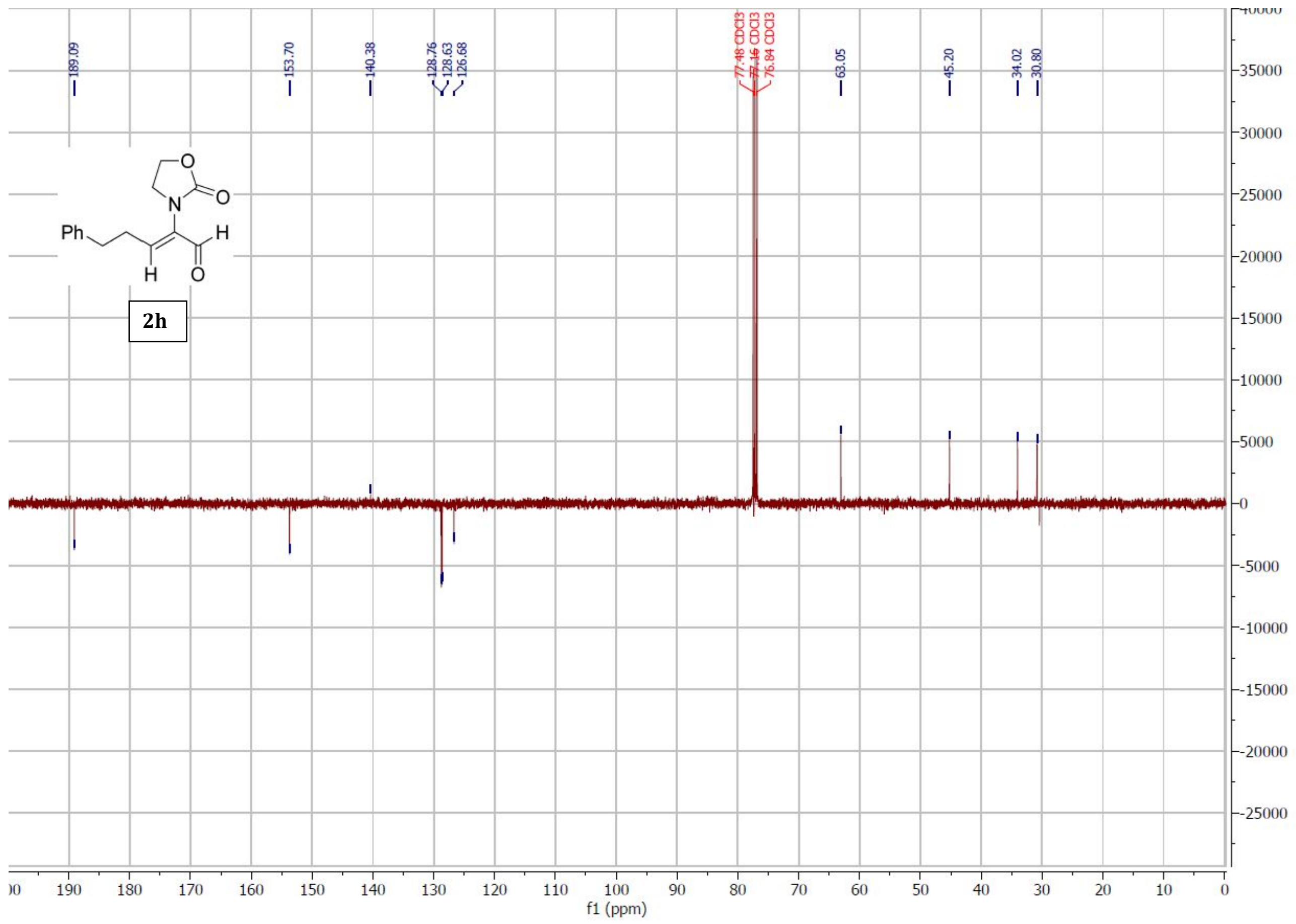


${ }^{1} \mathrm{H}$ NMR of compound $\mathbf{3 h}$ (400 MHz, $\mathrm{CDCl}_{3}$ ):

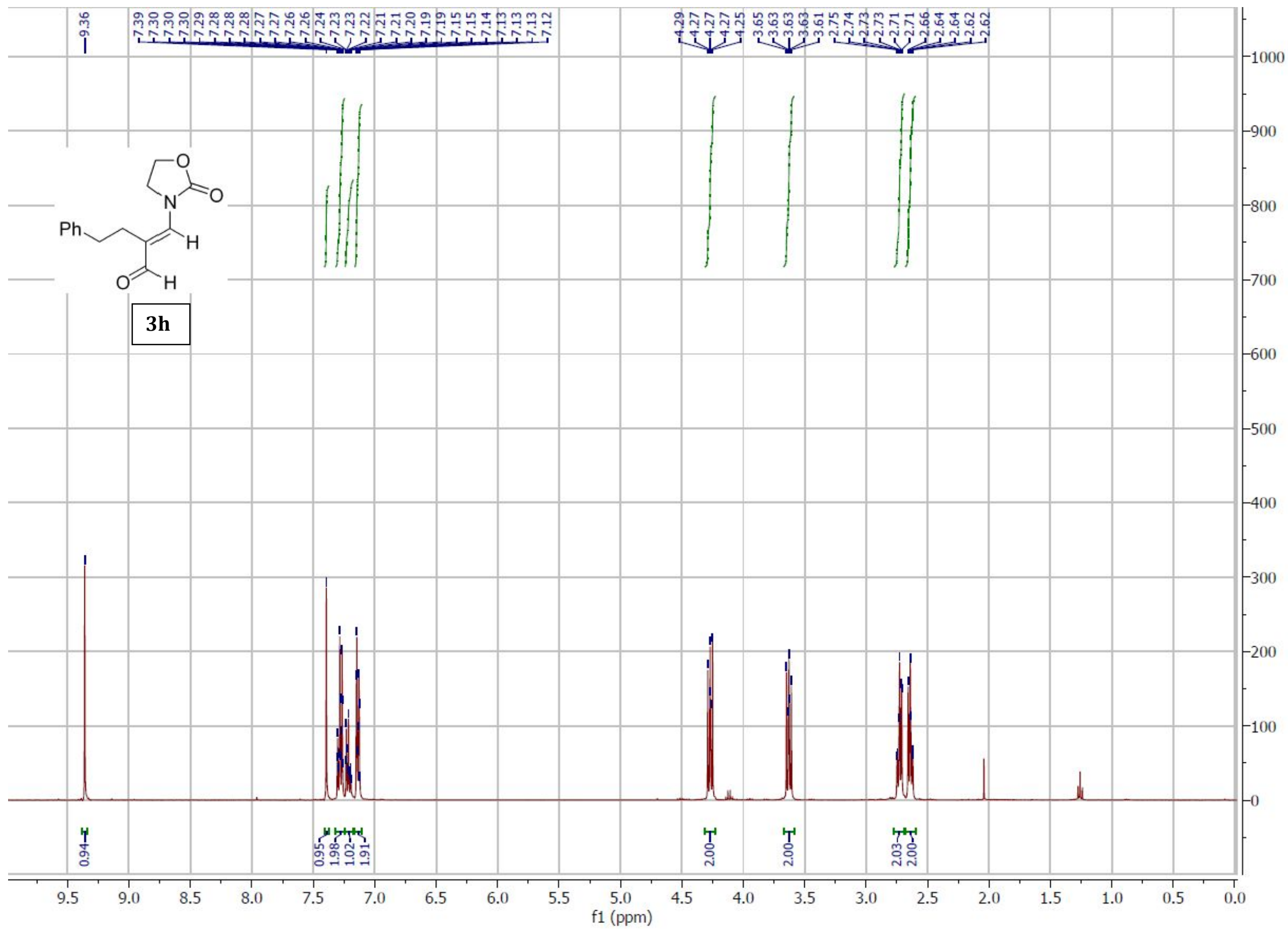


${ }^{13} \mathrm{C}$ NMR of compound $3 \mathbf{h}\left(101 \mathrm{MHz}, \mathrm{CDCl}_{3}\right)$ :

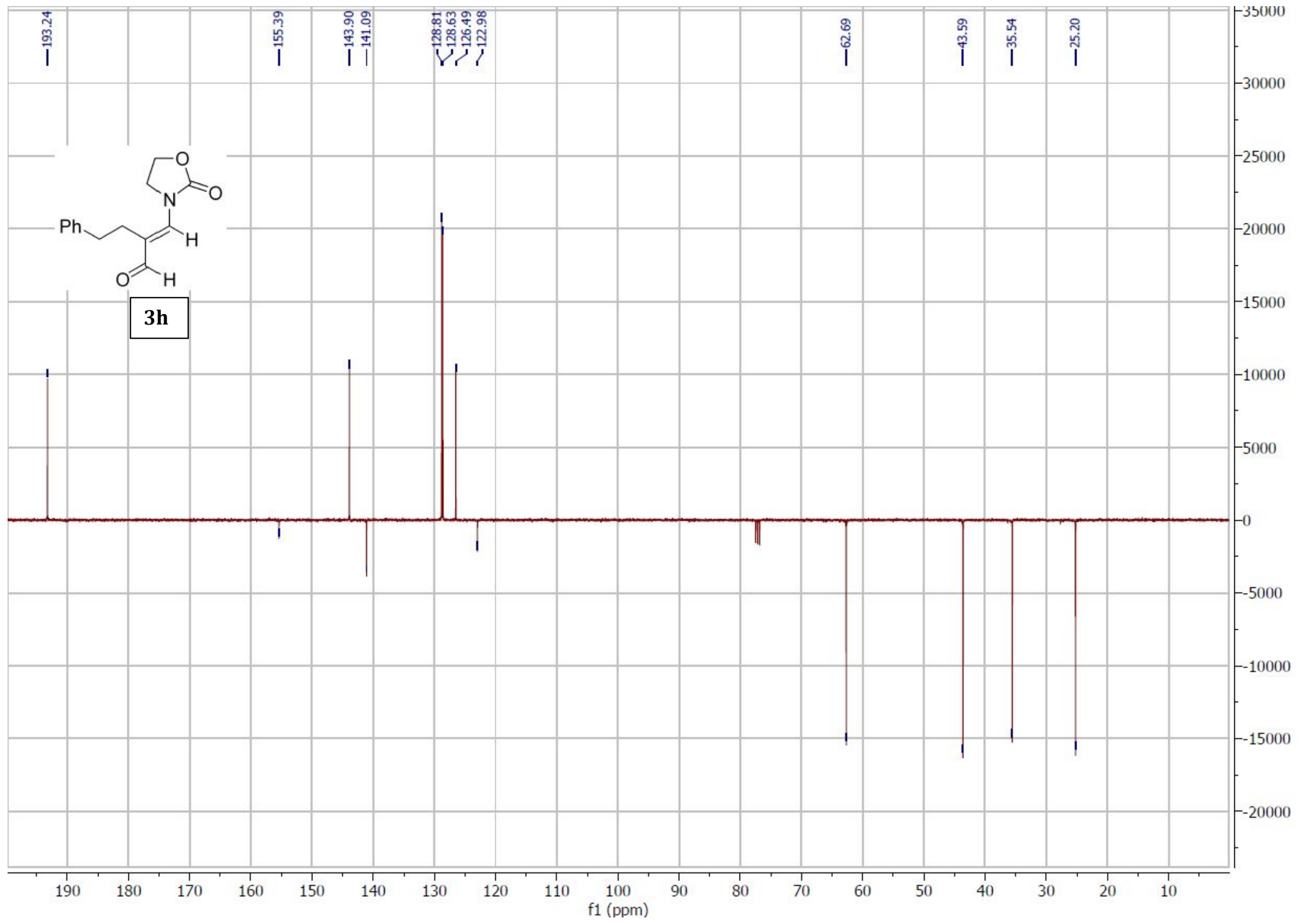


${ }^{1} \mathrm{H}$ NMR of compound $2 \mathbf{i}\left(500 \mathrm{MHz}, \mathrm{CDCl}_{3}\right.$ ):

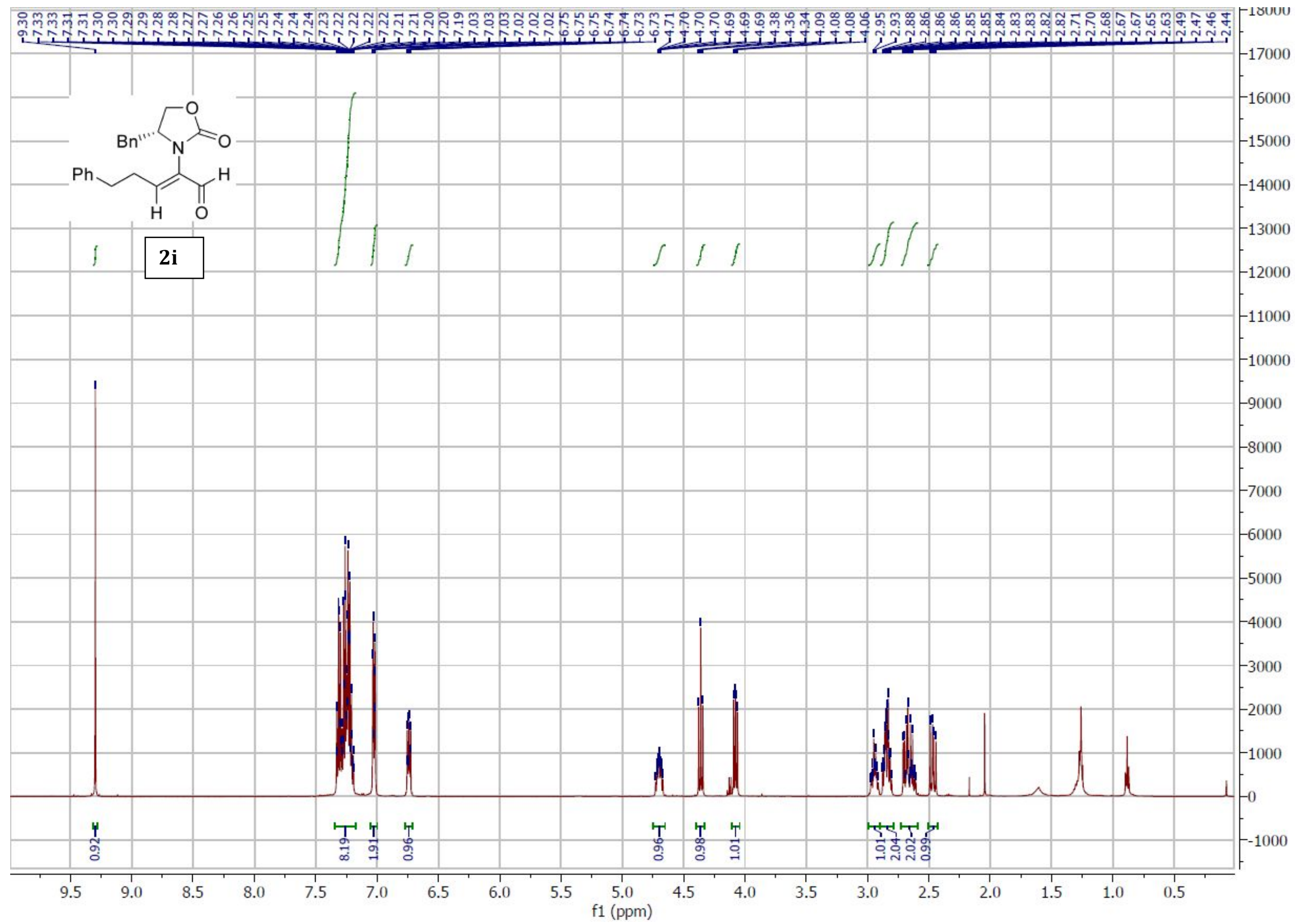


${ }^{13} \mathrm{C}$ NMR of compound $2 \mathbf{i}\left(126 \mathrm{MHz}, \mathrm{CDCl}_{3}\right)$ :

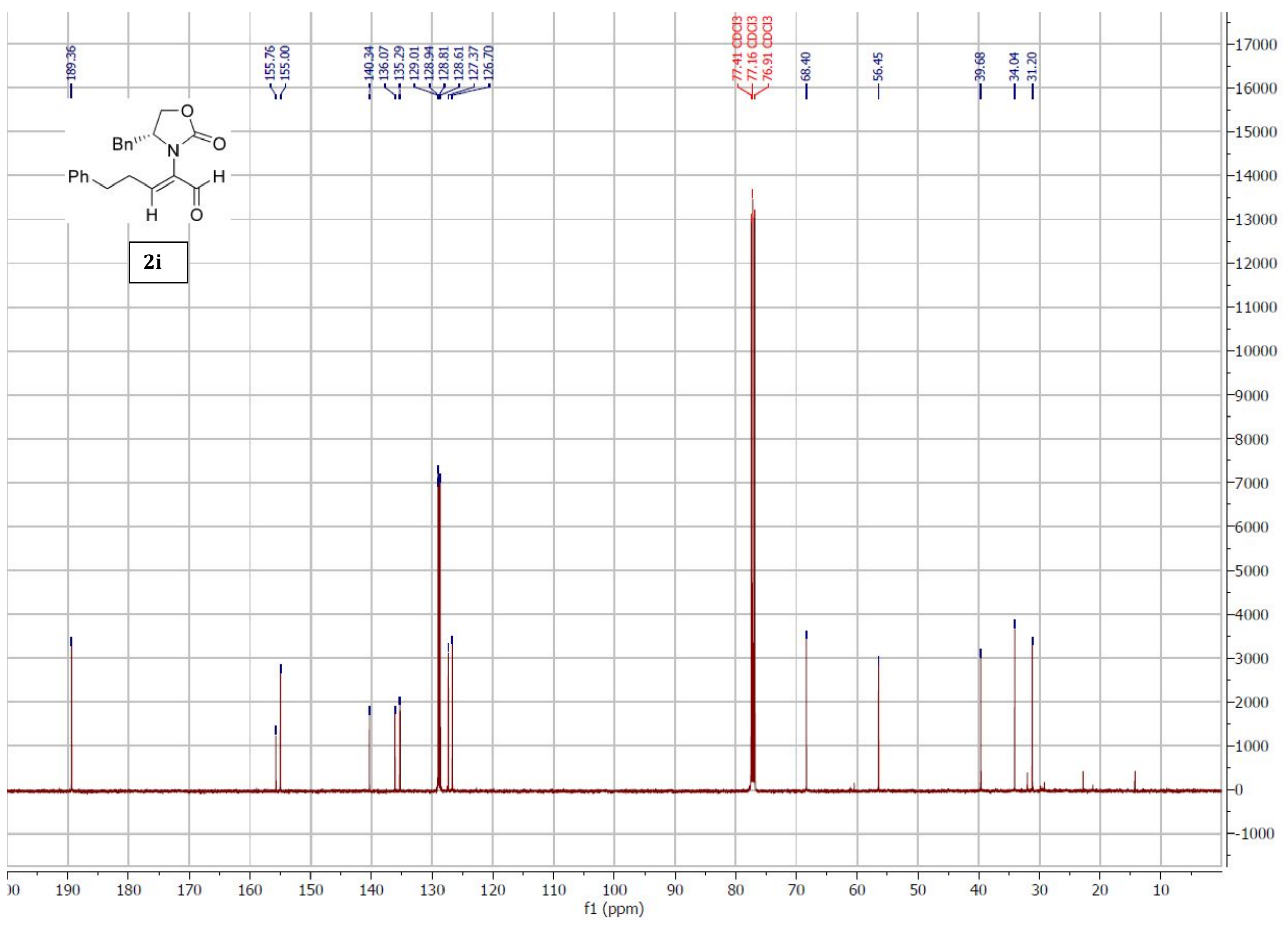


${ }^{1} \mathrm{H}$ NMR of compound $3 \mathbf{i}\left(500 \mathrm{MHz}, \mathrm{CDCl}_{3}\right)$ :

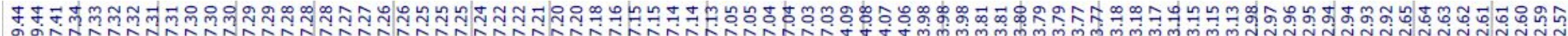

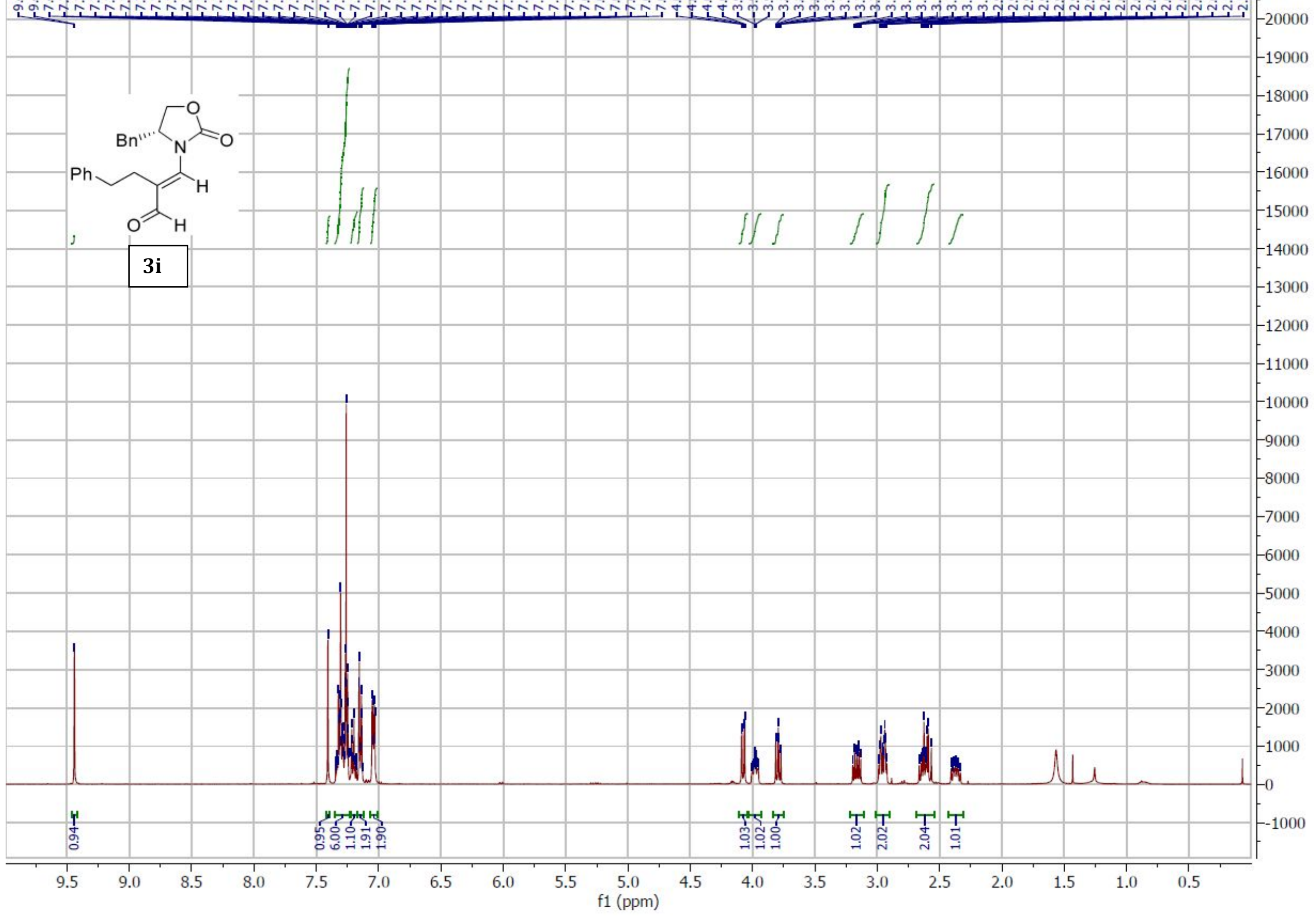


${ }^{13} \mathrm{C}$ NMR of compound 3i (126 MHz, $\left.\mathrm{CDCl}_{3}\right)$ :

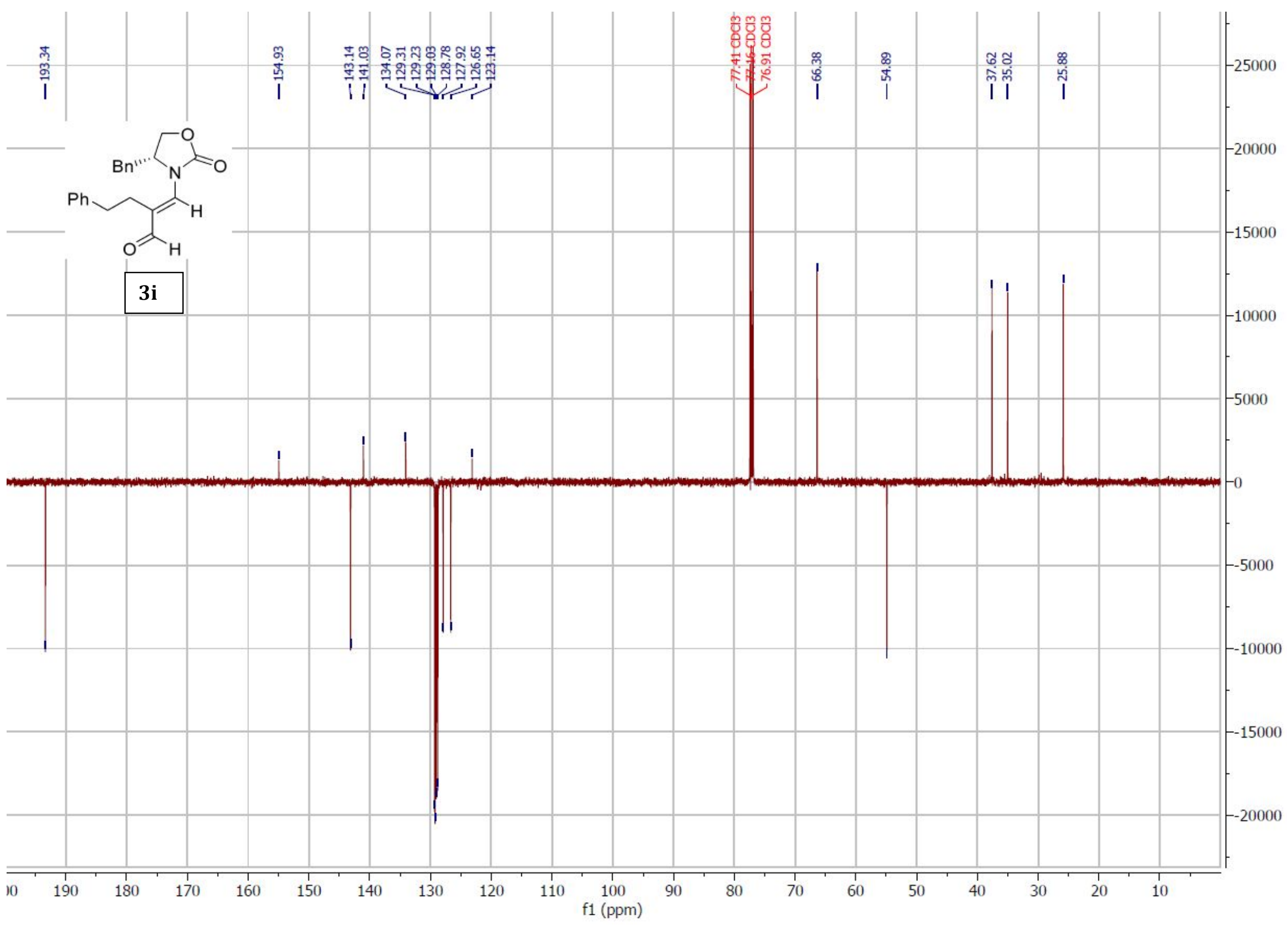


${ }^{1} \mathrm{H}$ NMR of compound $\mathbf{2} \mathbf{j}$ (400 MHz, $\mathrm{CDCl}_{3}$ ):

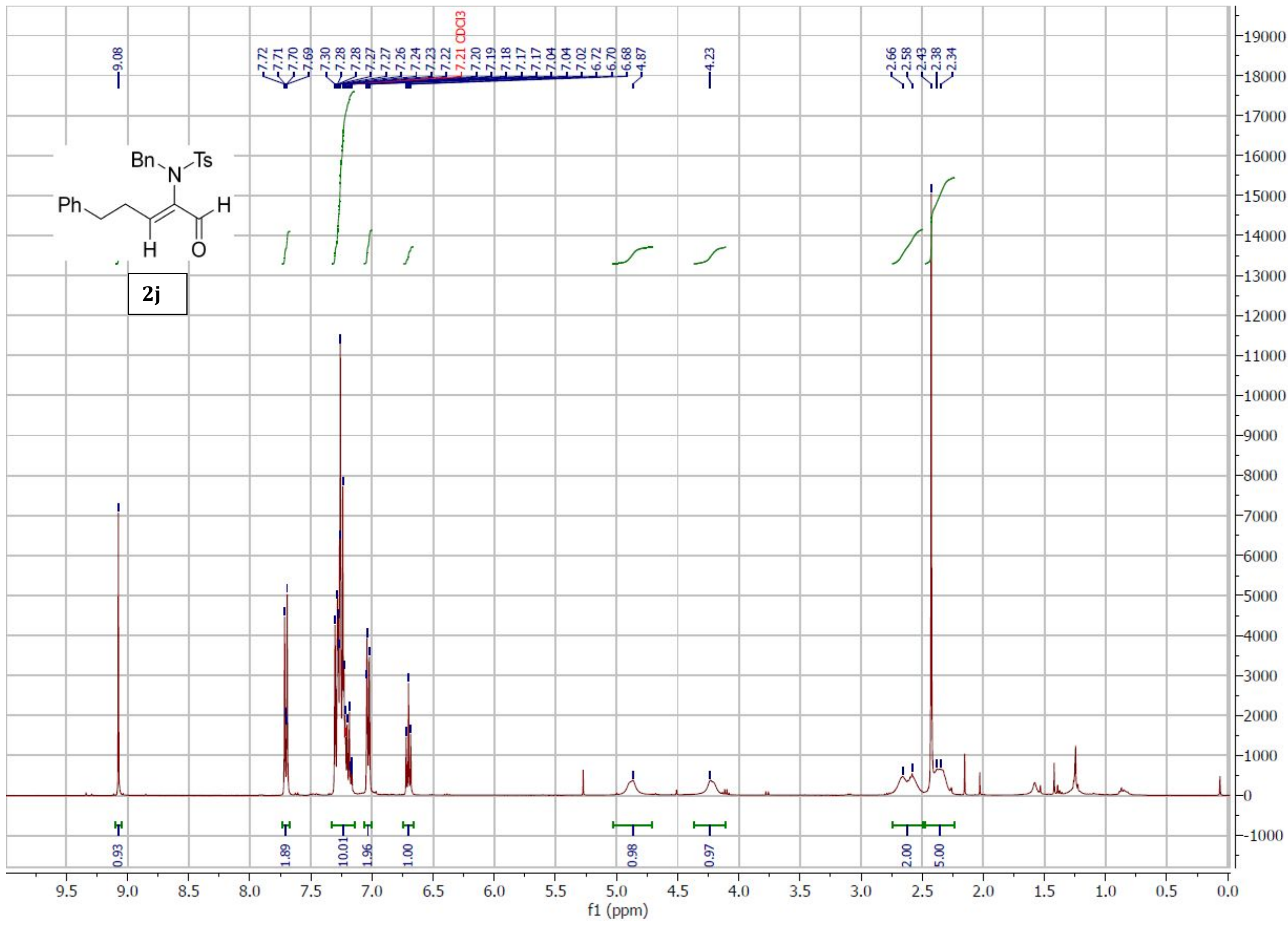


${ }^{13} \mathrm{C}$ NMR of compound $\mathbf{2 j}$ (101 MHz, $\mathrm{CDCl}_{3}$ ):

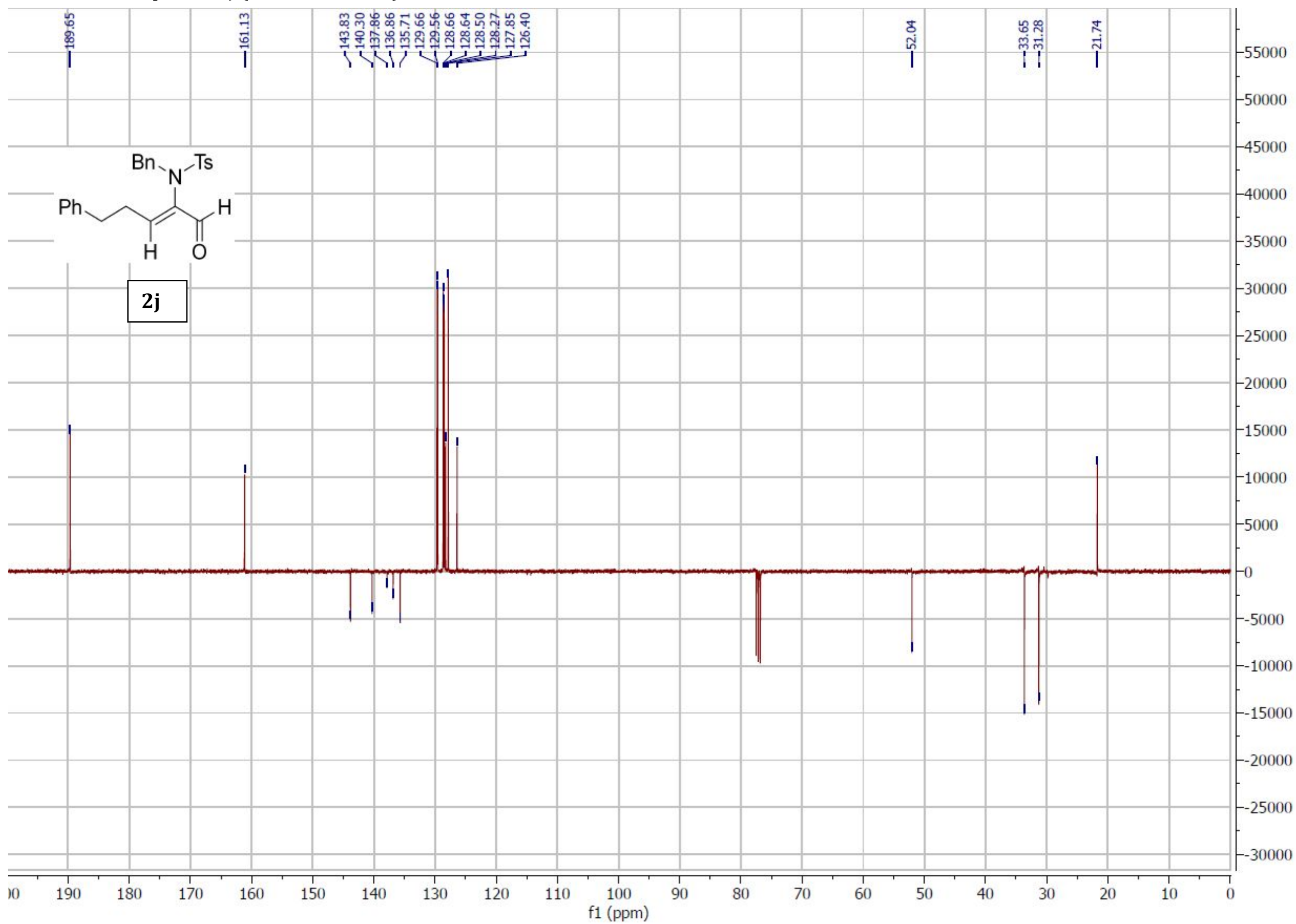


${ }^{1} \mathrm{H}$ NMR of compound $3 \mathbf{j}\left(400 \mathrm{MHz}, \mathrm{CDCl}_{3}\right.$ ):

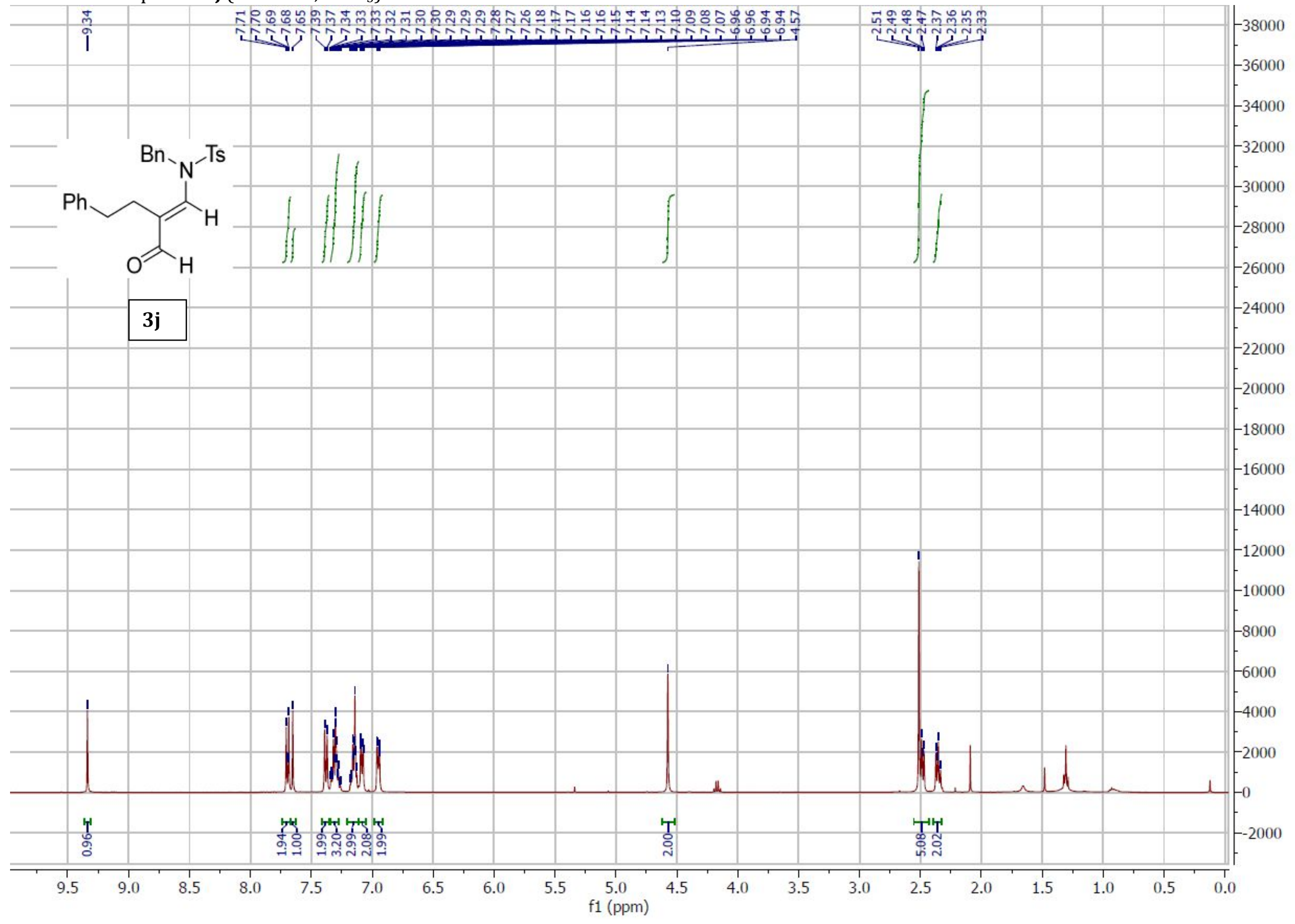


${ }^{13} \mathrm{C}$ NMR of compound 3j (101 MHz, $\left.\mathrm{CDCl}_{3}\right)$ :

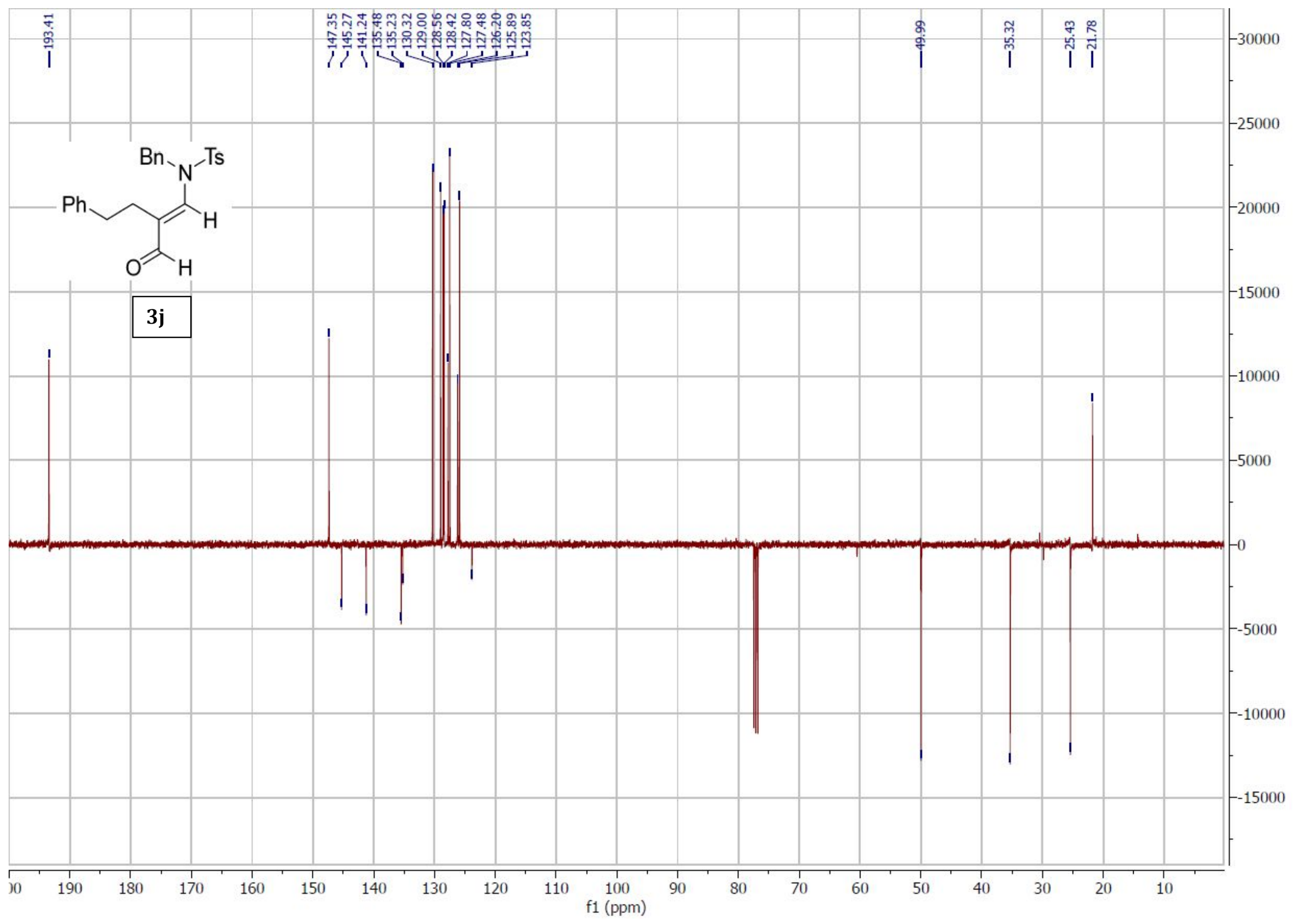


${ }^{1} \mathrm{H}$ NMR of compound $\mathbf{2 k}\left(500 \mathrm{MHz}, \mathrm{CDCl}_{3}\right)$ :

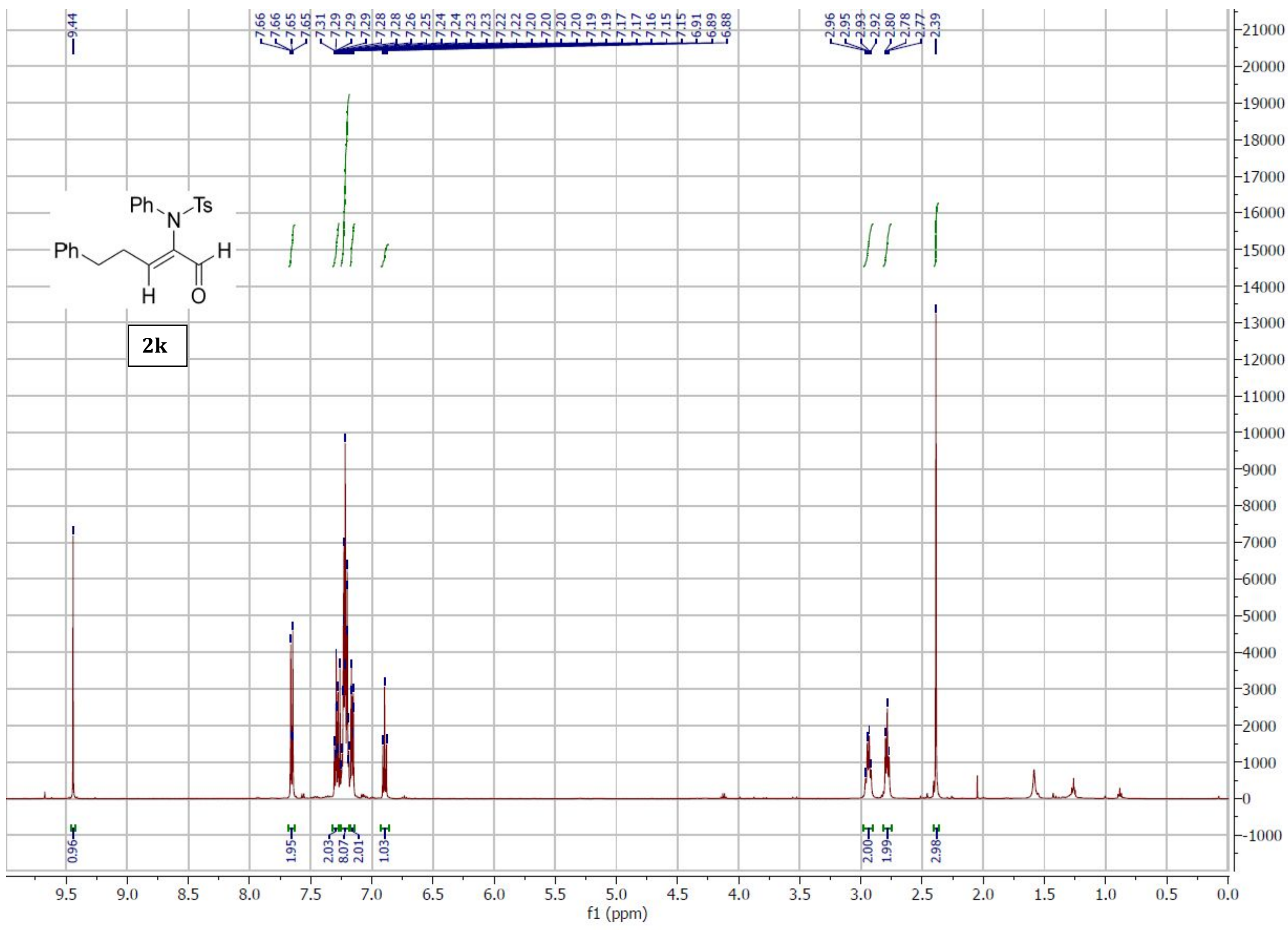


${ }^{13} \mathrm{C}$ NMR of compound $\mathbf{2 k}\left(126 \mathrm{MHz}, \mathrm{CDCl}_{3}\right)$ :

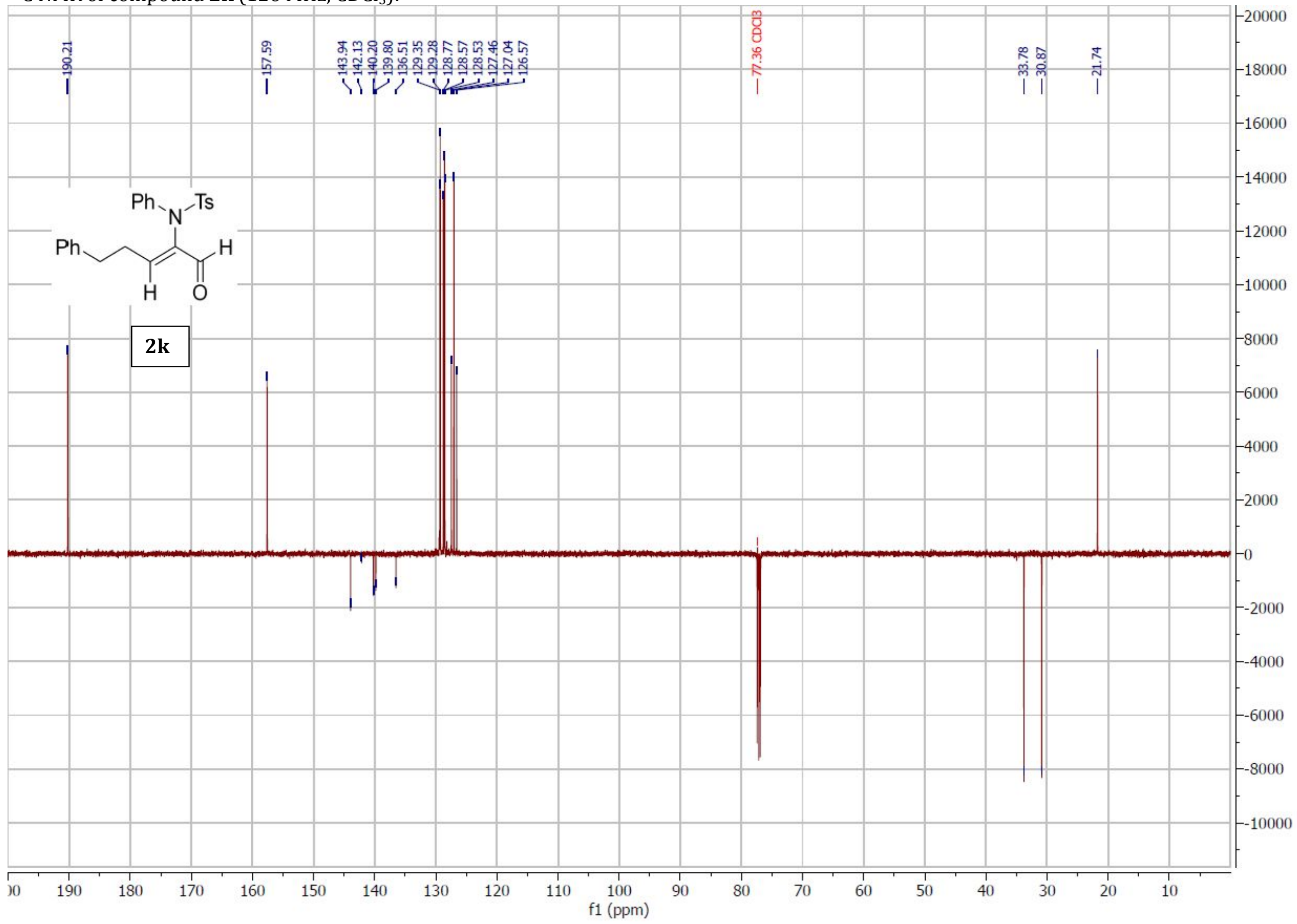


${ }^{1} \mathrm{H}$ NMR of compound 3k (500 MHz, $\left.\mathrm{CDCl}_{3}\right)$ :

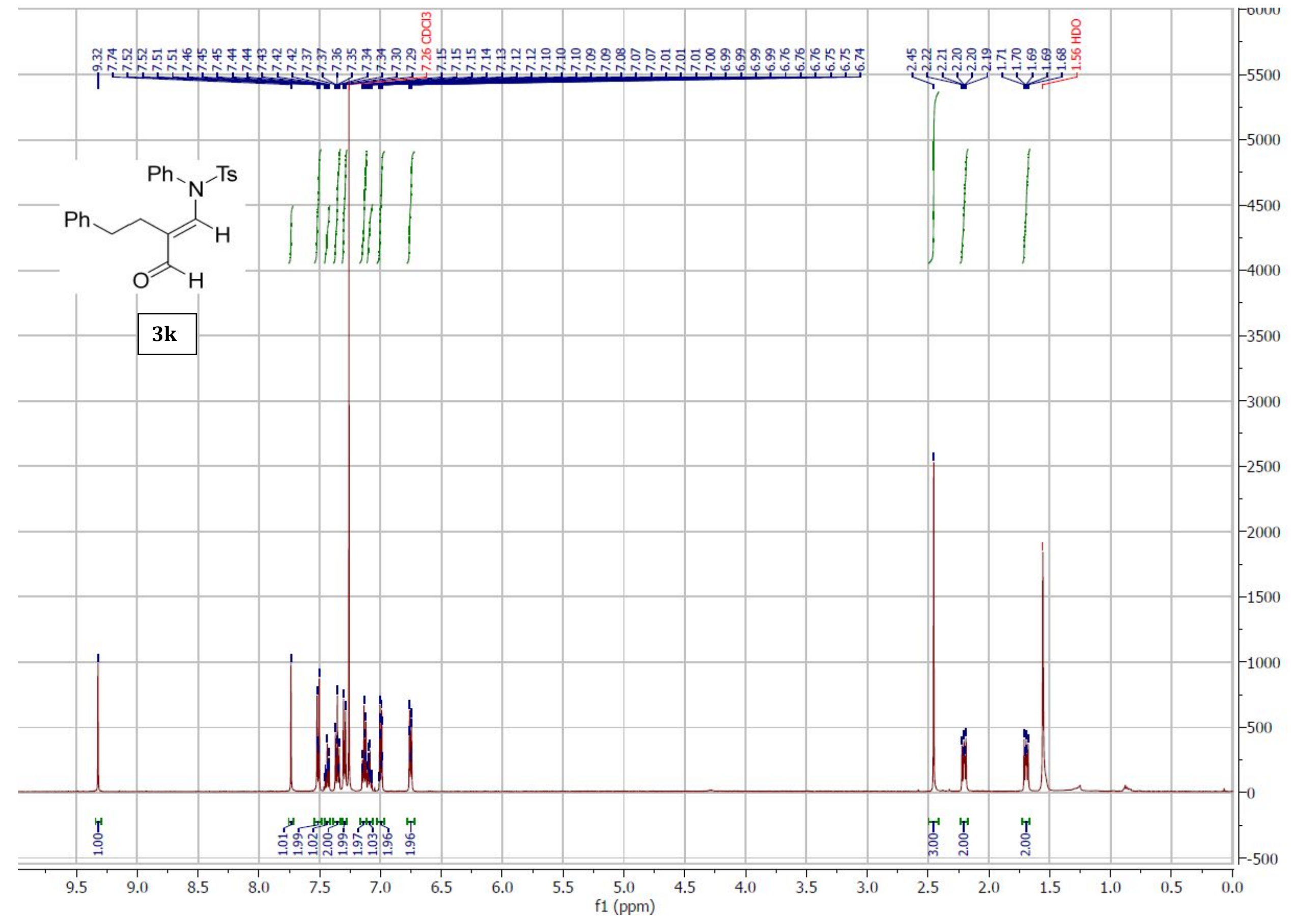


${ }^{13} \mathrm{C}$ NMR of compound 3k (126 MHz, $\left.\mathrm{CDCl}_{3}\right)$ :

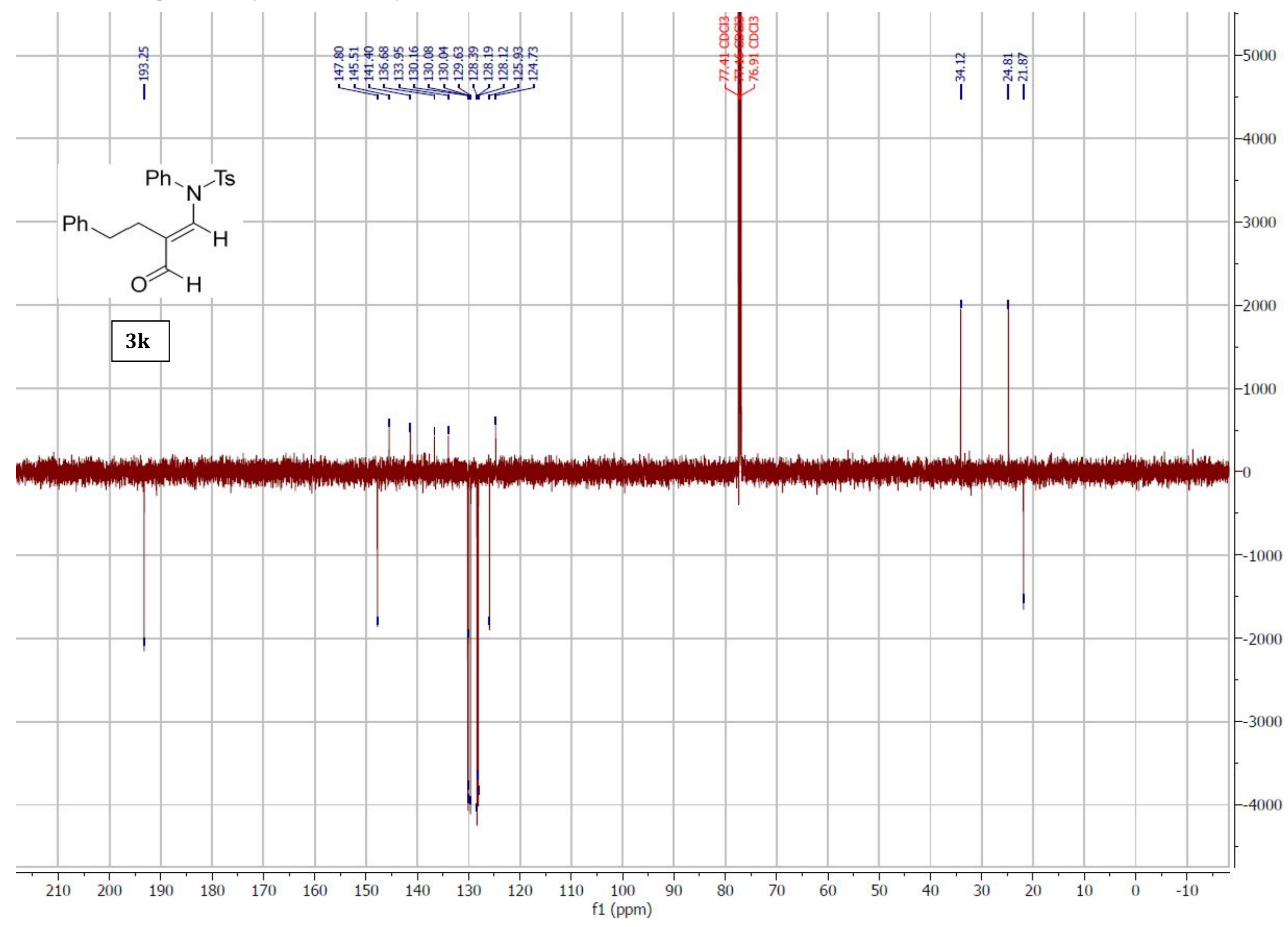


${ }^{1} \mathrm{H}$ NMR of compound $\mathbf{2 m}\left(400 \mathrm{MHz}, \mathrm{CDCl}_{3}\right)$ :

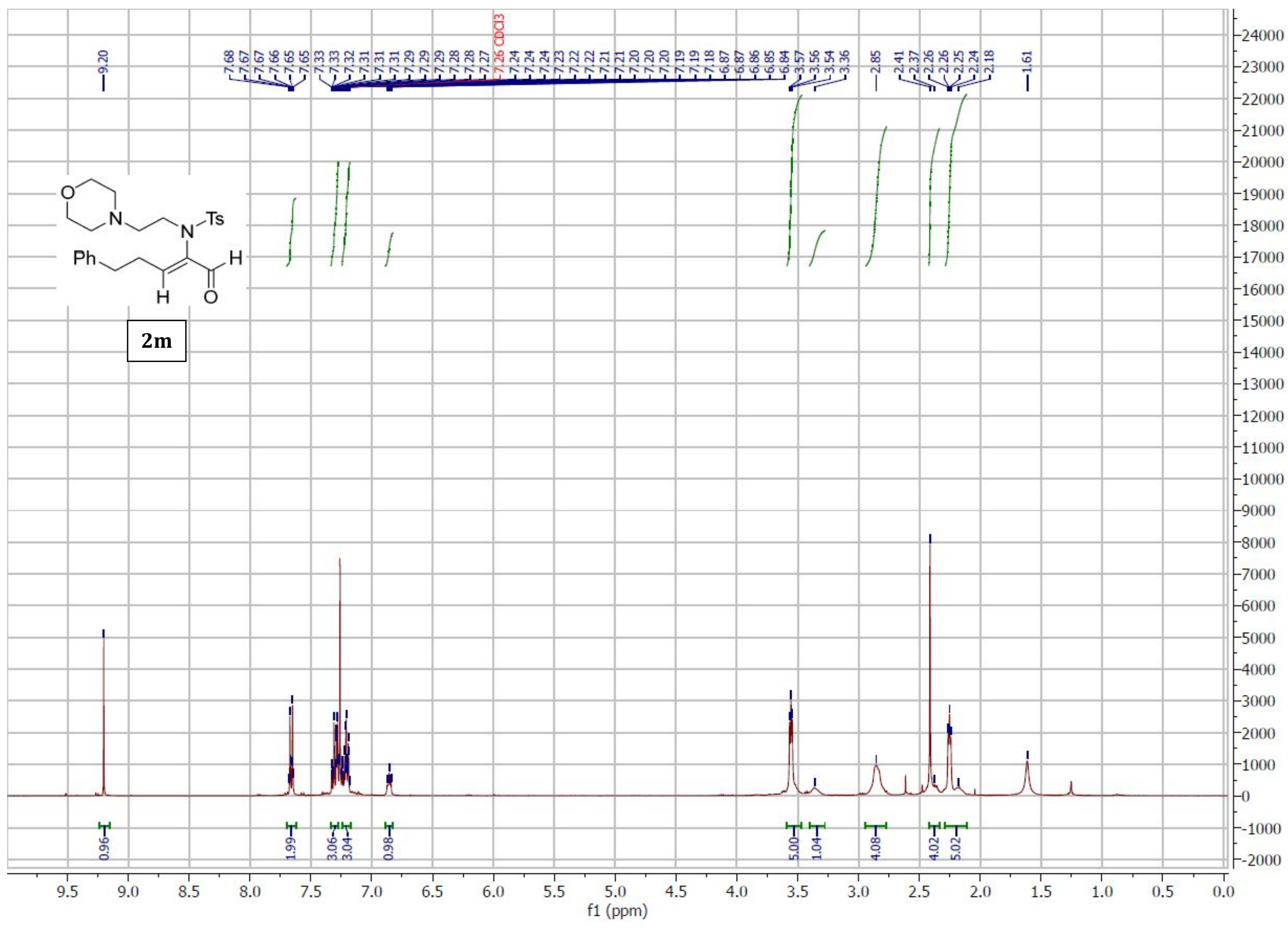


${ }^{13} \mathrm{C}$ NMR of compound $\mathbf{2 m}\left(101 \mathrm{MHz}, \mathrm{CDCl}_{3}\right)$ :

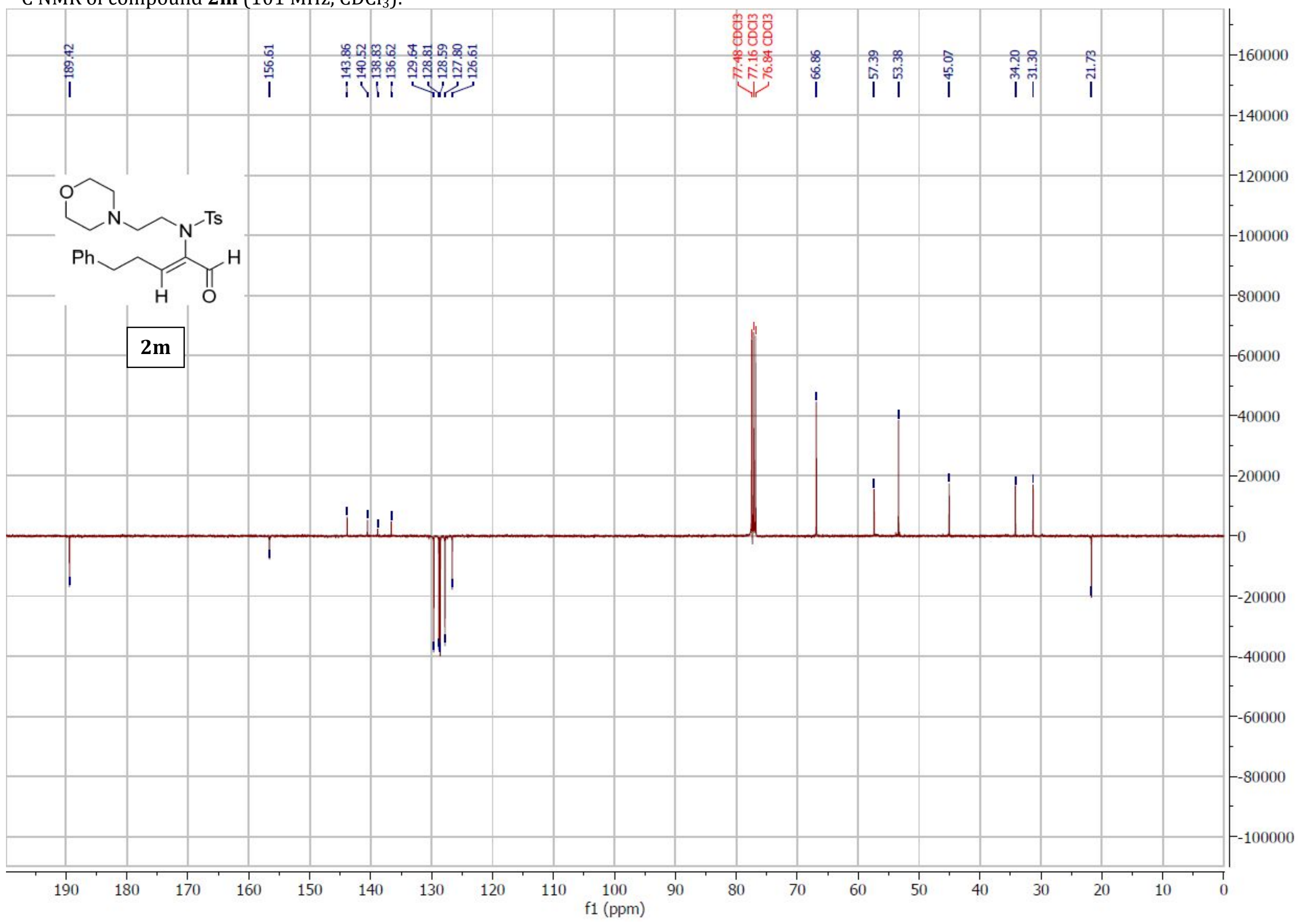


${ }^{1} \mathrm{H}$ NMR of compound $3 \mathbf{m}\left(400 \mathrm{MHz}, \mathrm{CDCl}_{3}\right)$ :

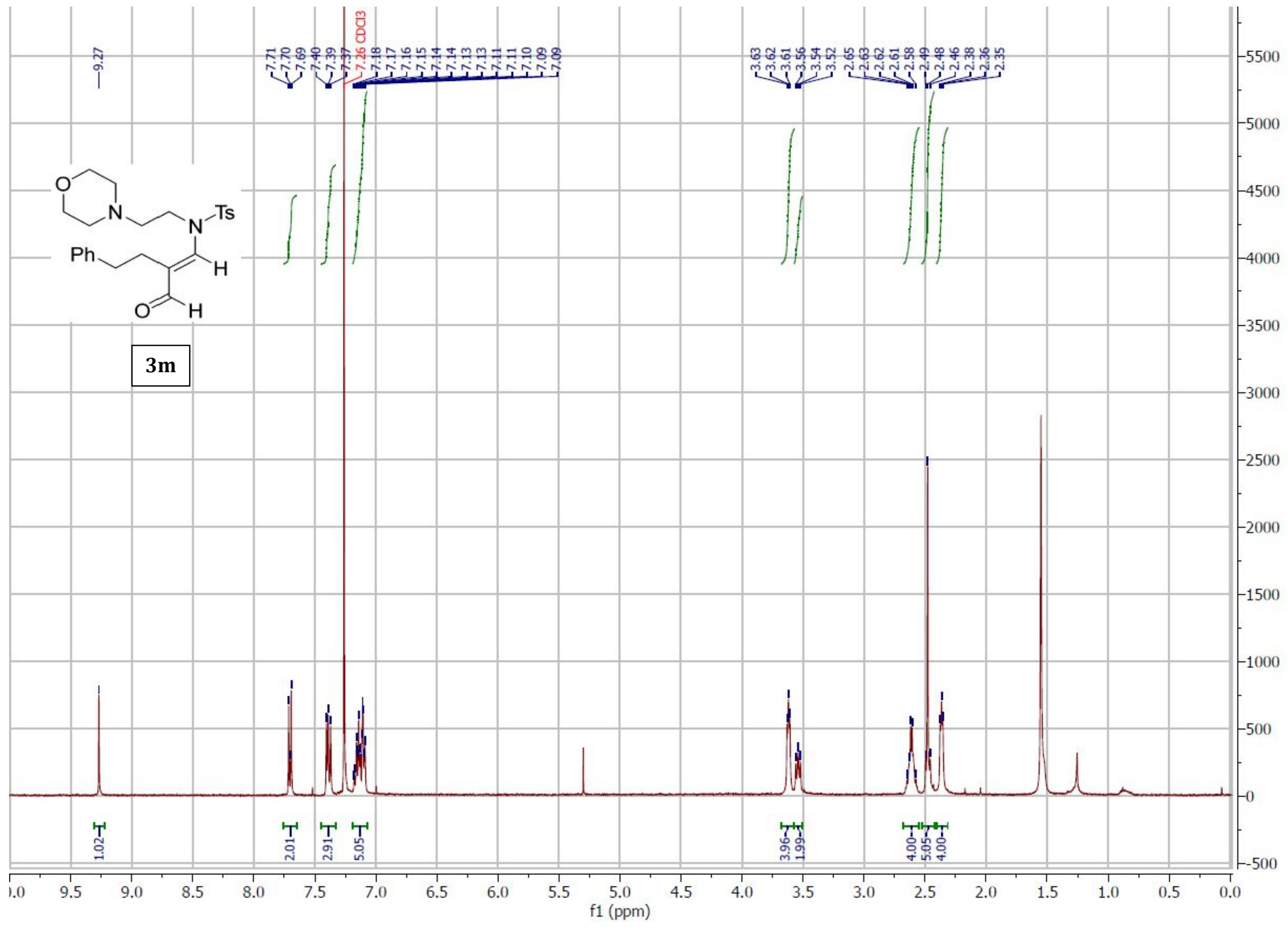


${ }^{13} \mathrm{C}$ NMR of compound $3 \mathbf{m}\left(101 \mathrm{MHz}, \mathrm{CDCl}_{3}\right)$ :

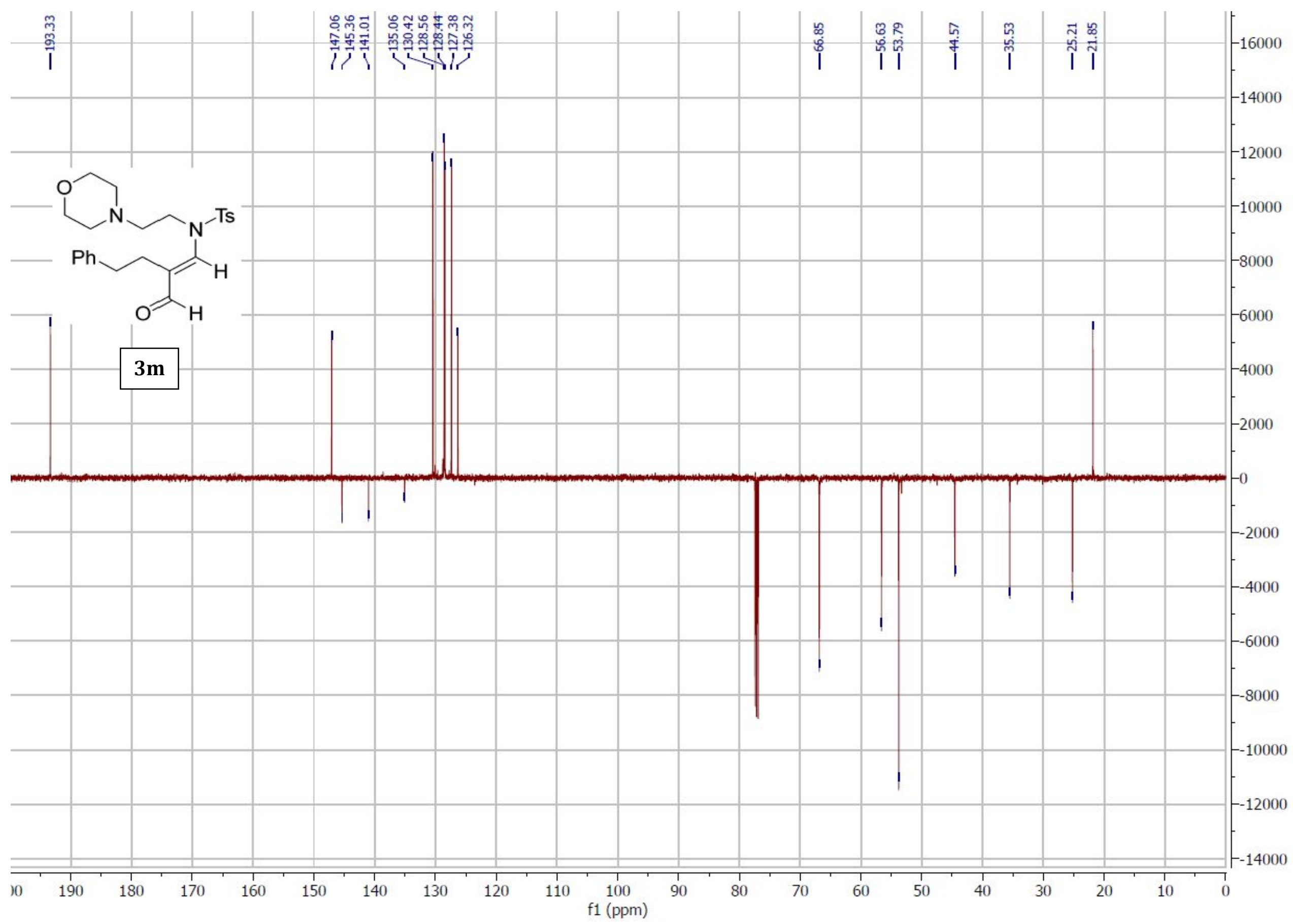


${ }^{1} \mathrm{H}$ NMR of compound 2 n (400 MHz, $\left.\mathrm{CDCl}_{3}\right)$ :

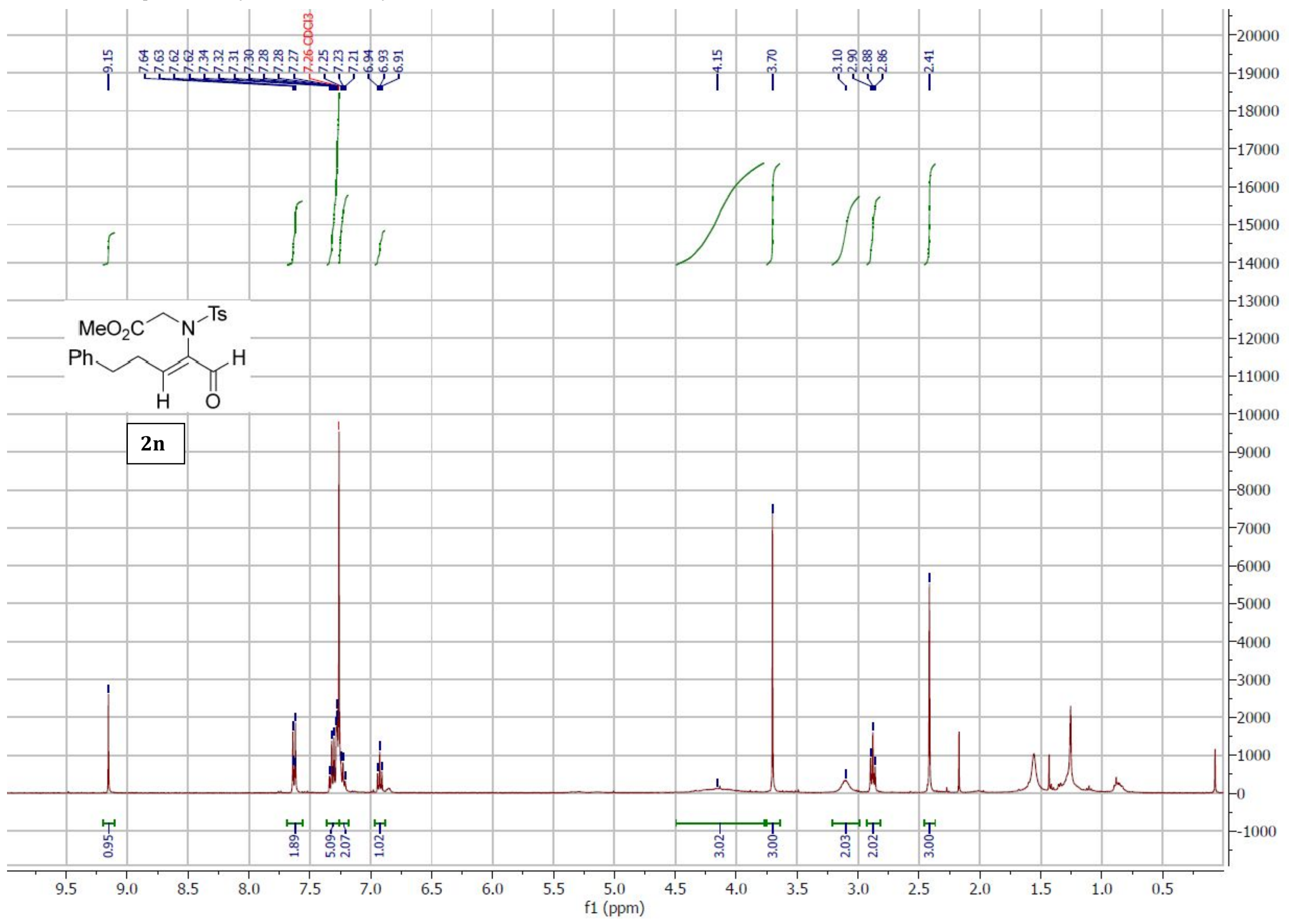


${ }^{13} \mathrm{C}$ NMR of compound $2 \mathbf{n}\left(101 \mathrm{MHz}, \mathrm{CDCl}_{3}\right)$ :

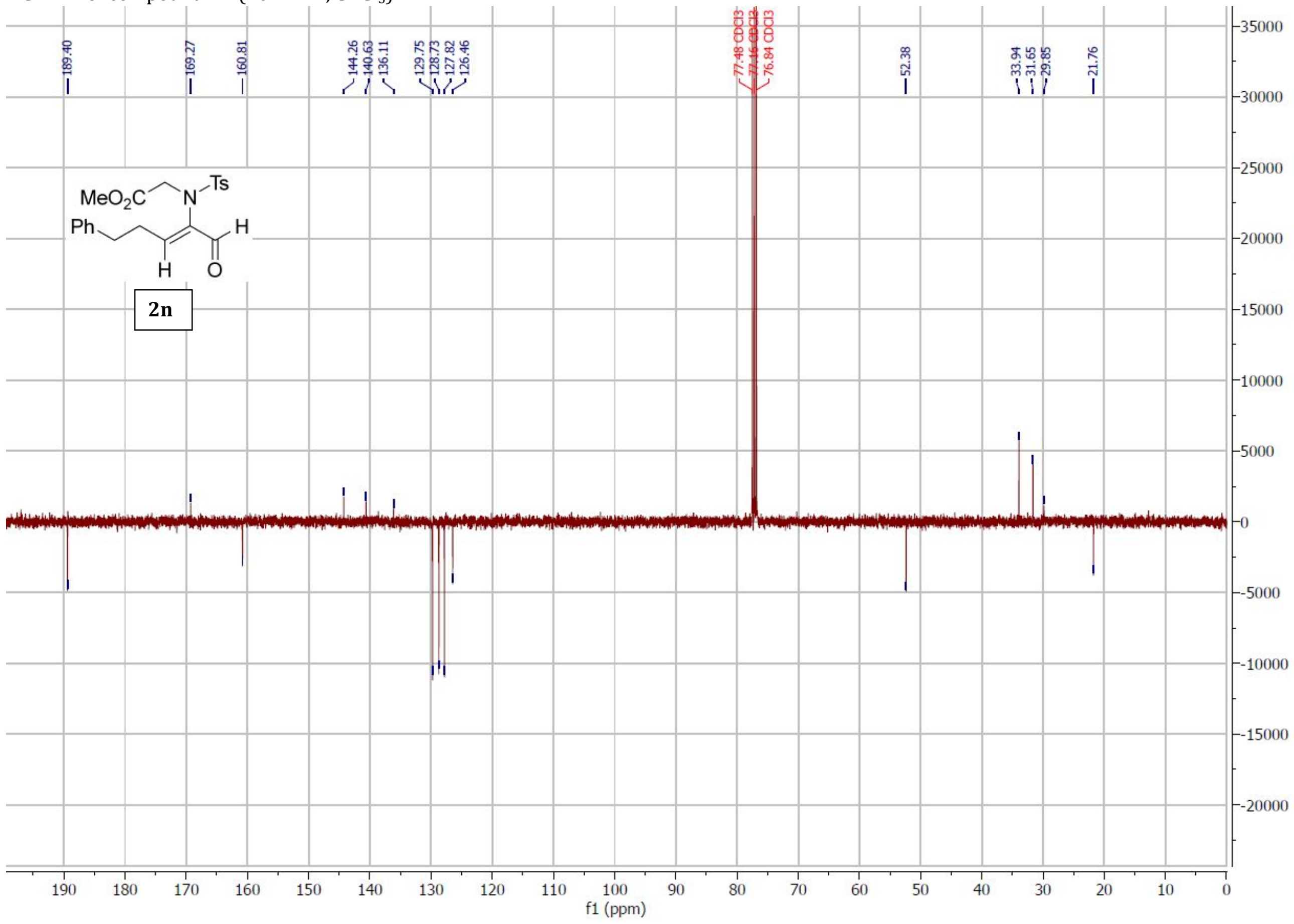


${ }^{1} \mathrm{H}$ NMR of compound $3 \mathbf{n}\left(400 \mathrm{MHz}, \mathrm{CDCl}_{3}\right)$ :

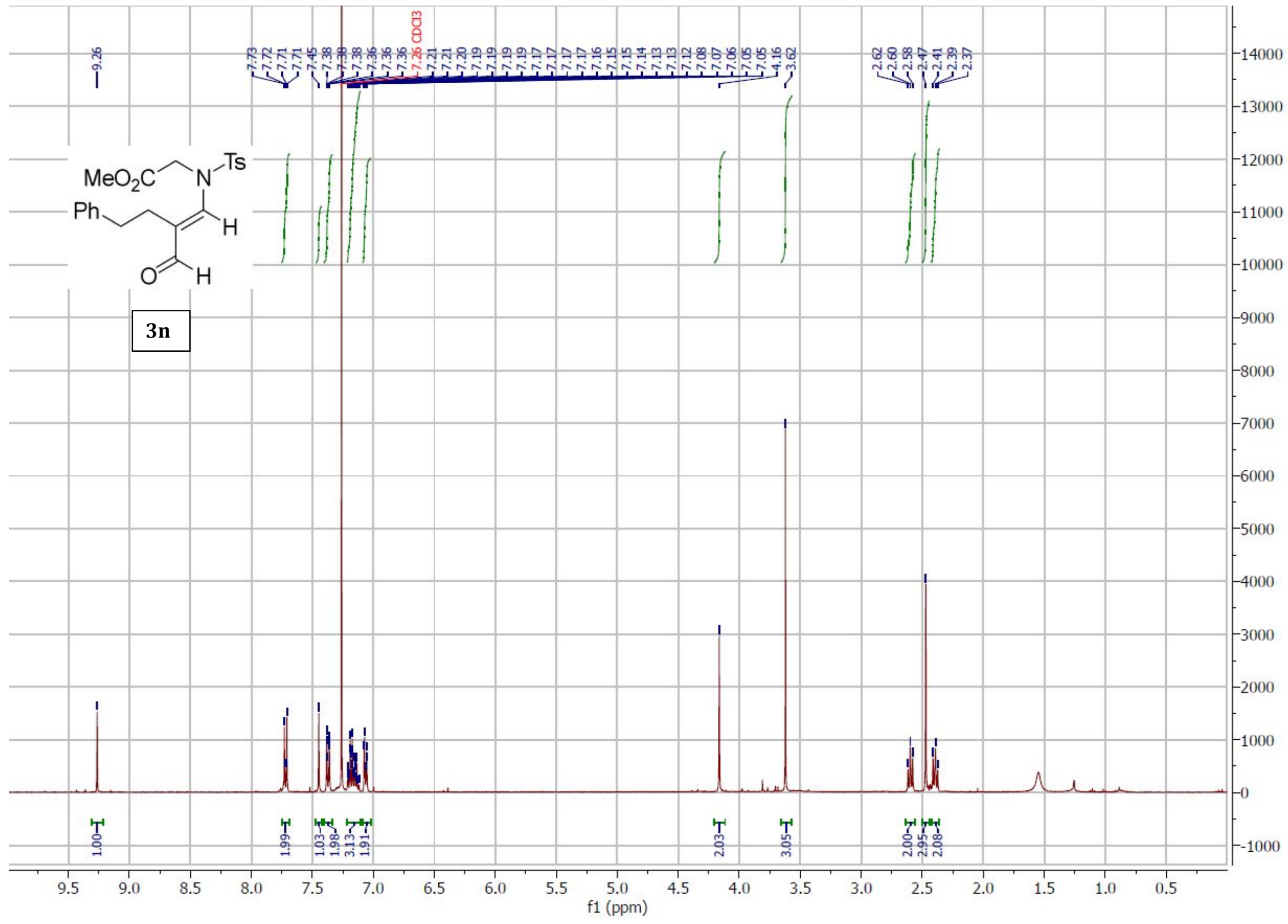


${ }^{13} \mathrm{C}$ NMR of compound 3n (101 MHz, $\left.\mathrm{CDCl}_{3}\right)$ :

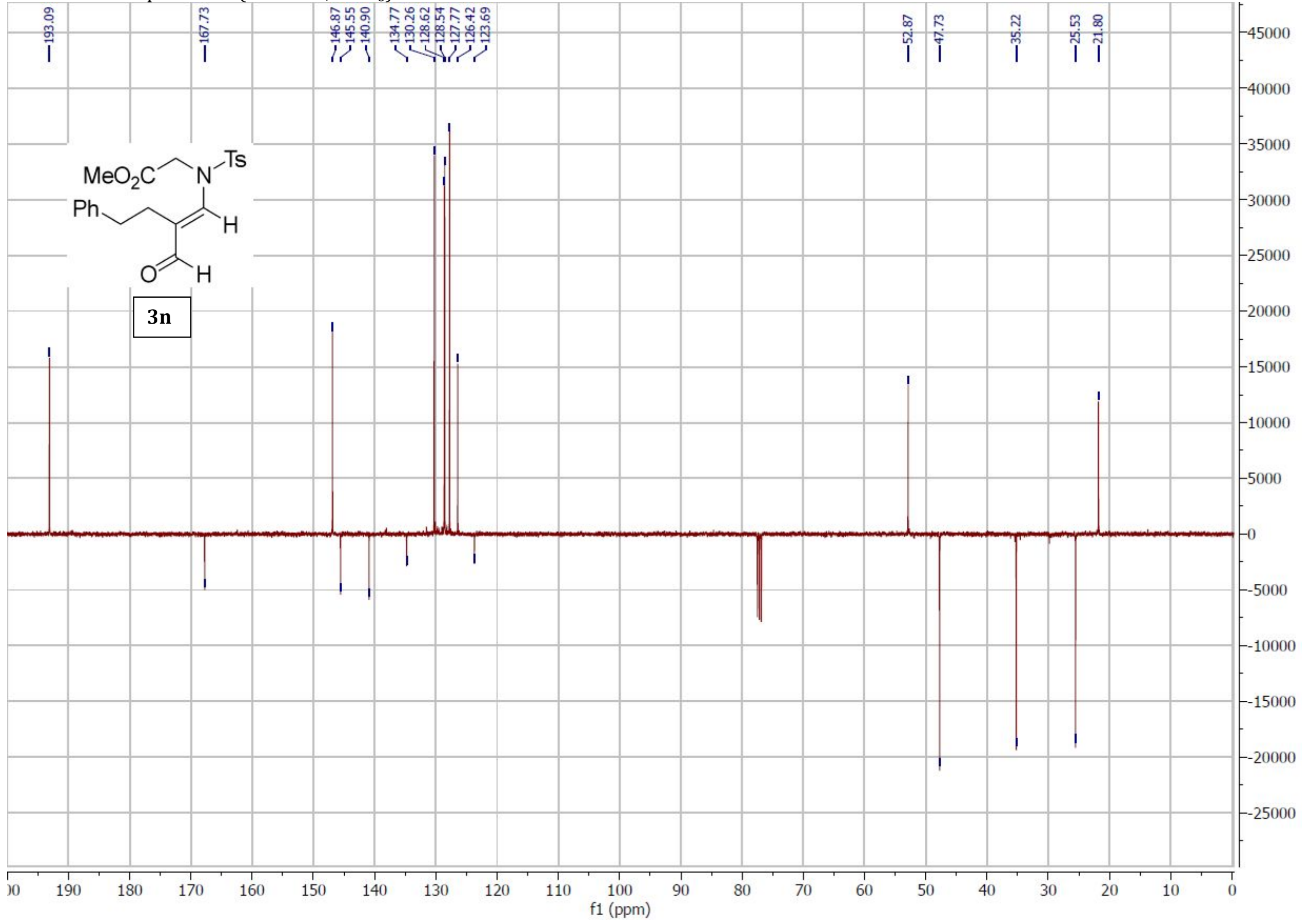


${ }^{1} \mathrm{H}$ NMR of compound $20\left(400 \mathrm{MHz}, \mathrm{CDCl}_{3}\right)$ :

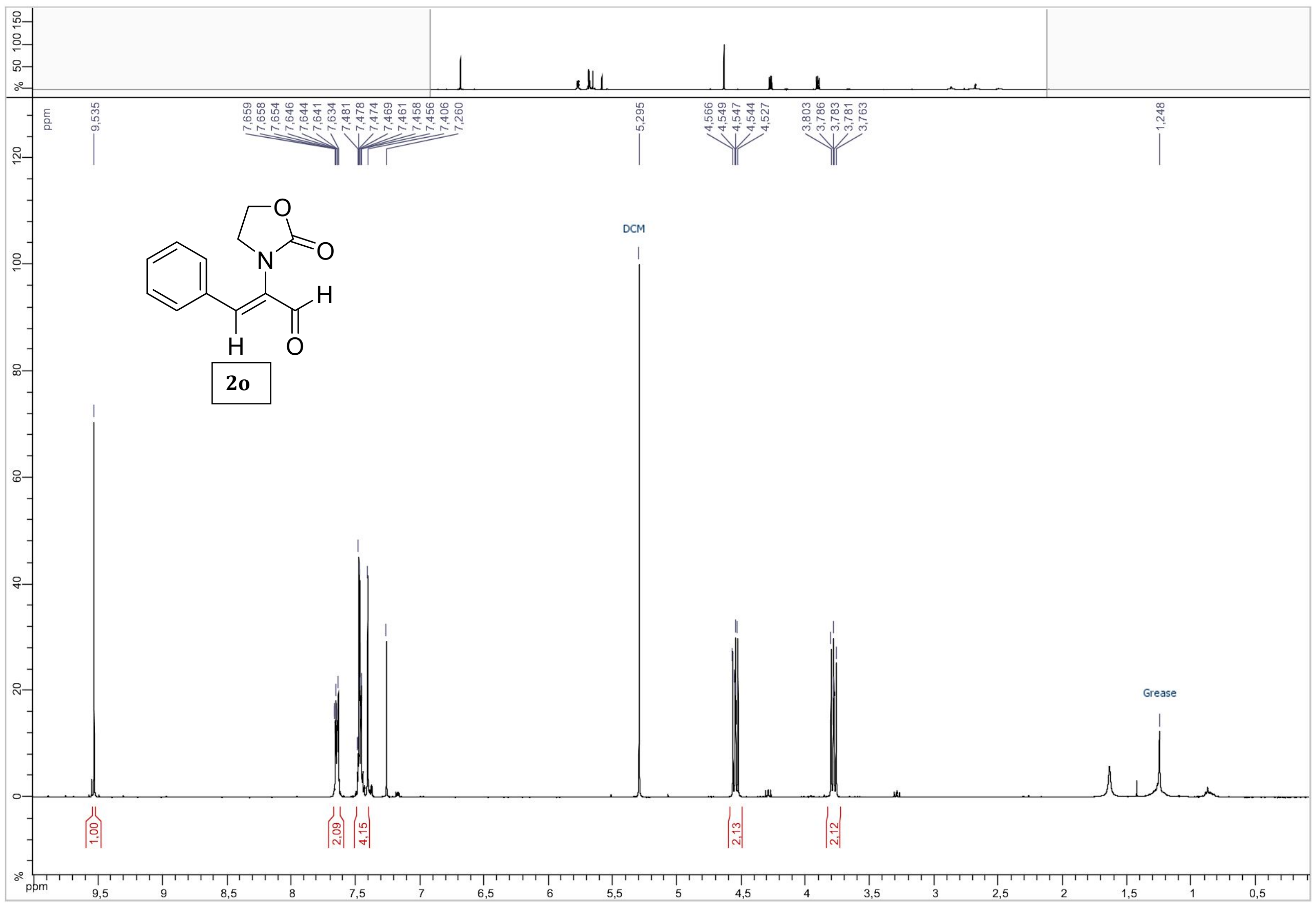


${ }^{13} \mathrm{C}$ NMR of compound 2 o (101 MHz, $\left.\mathrm{CDCl}_{3}\right)$ :

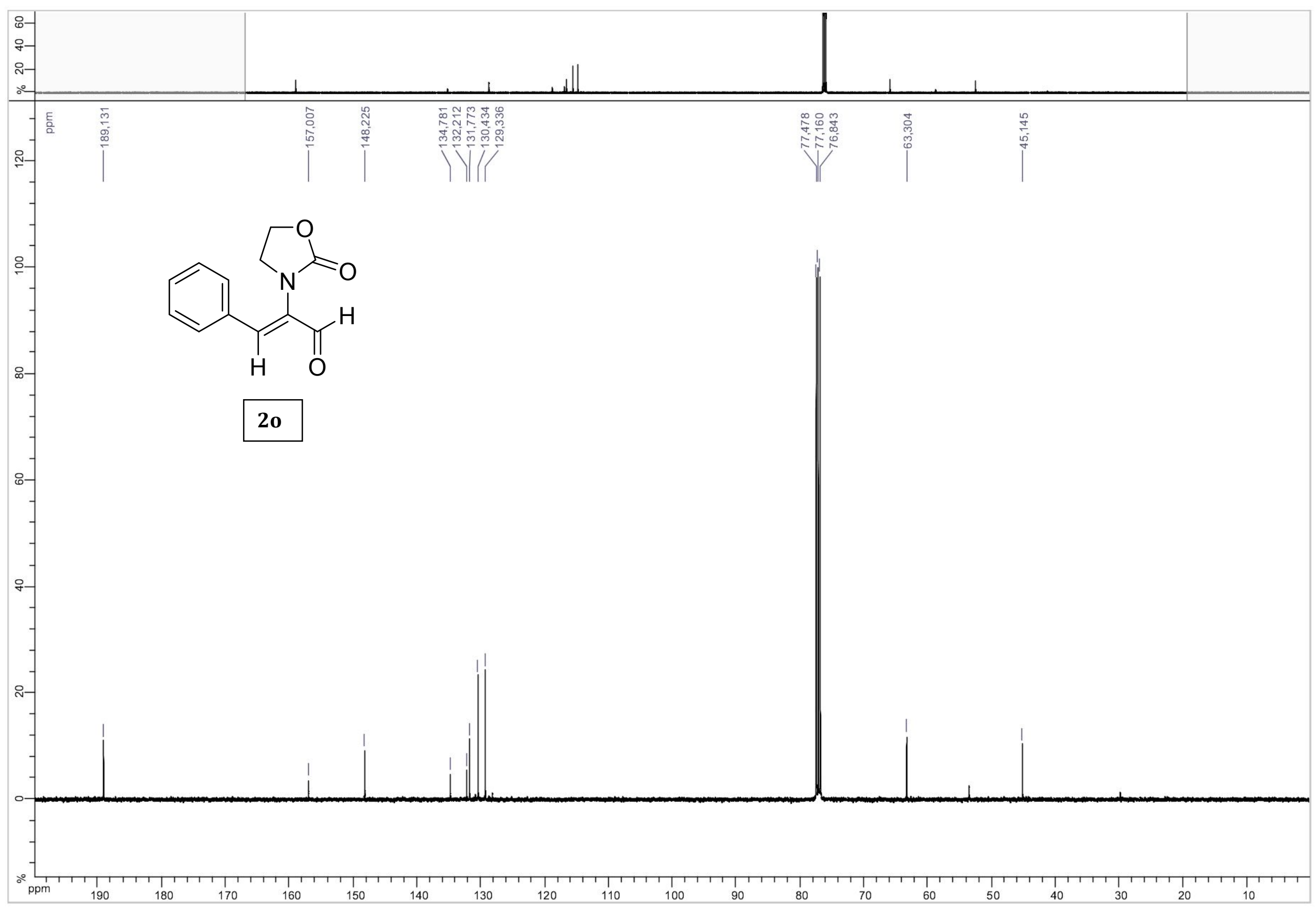


${ }^{1} \mathrm{H}$ NMR of compound $30\left(400 \mathrm{MHz}, \mathrm{CDCl}_{3}\right)$ :

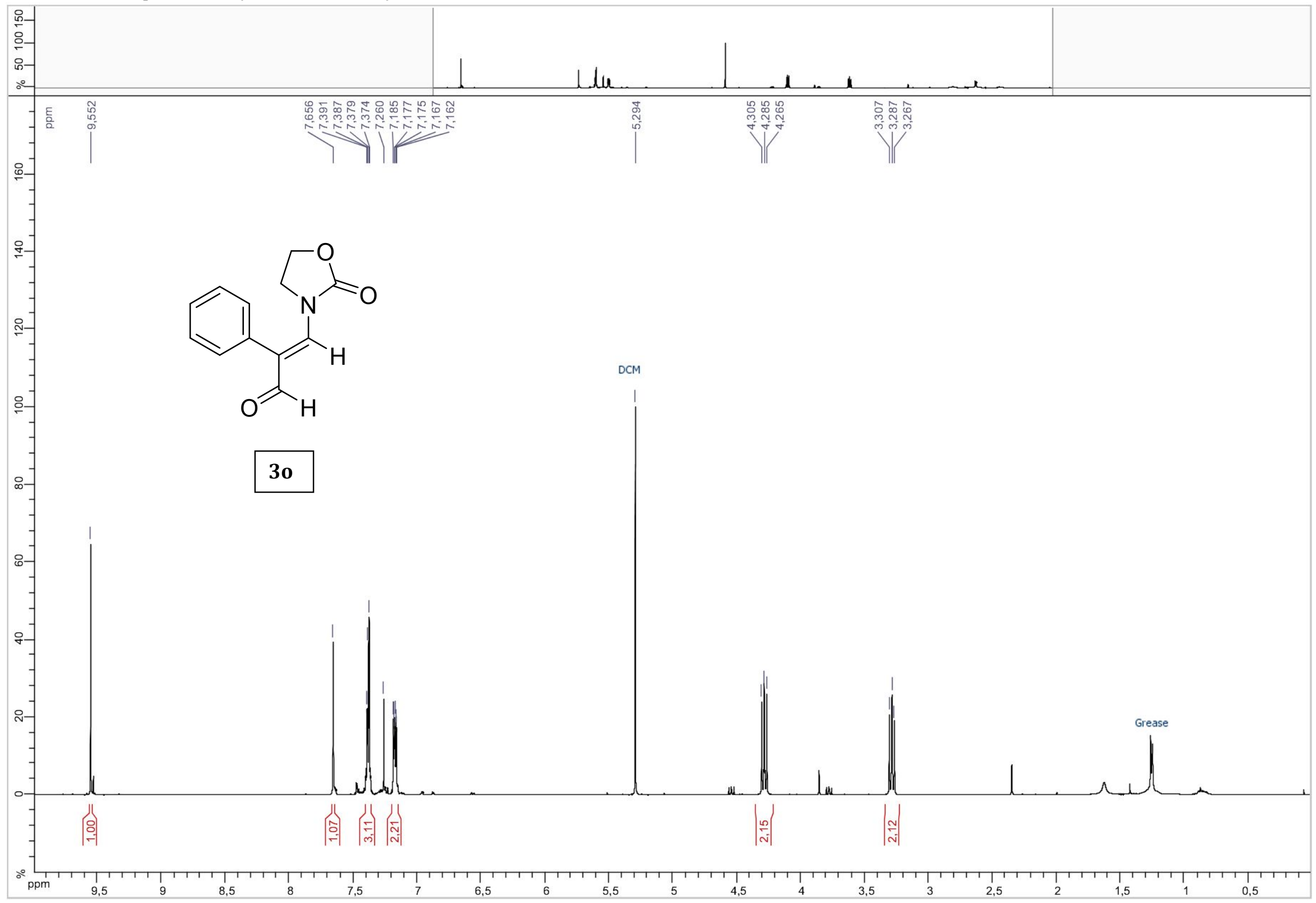


${ }^{13} \mathrm{C}$ NMR of compound $30\left(101 \mathrm{MHz}, \mathrm{CDCl}_{3}\right)$ :

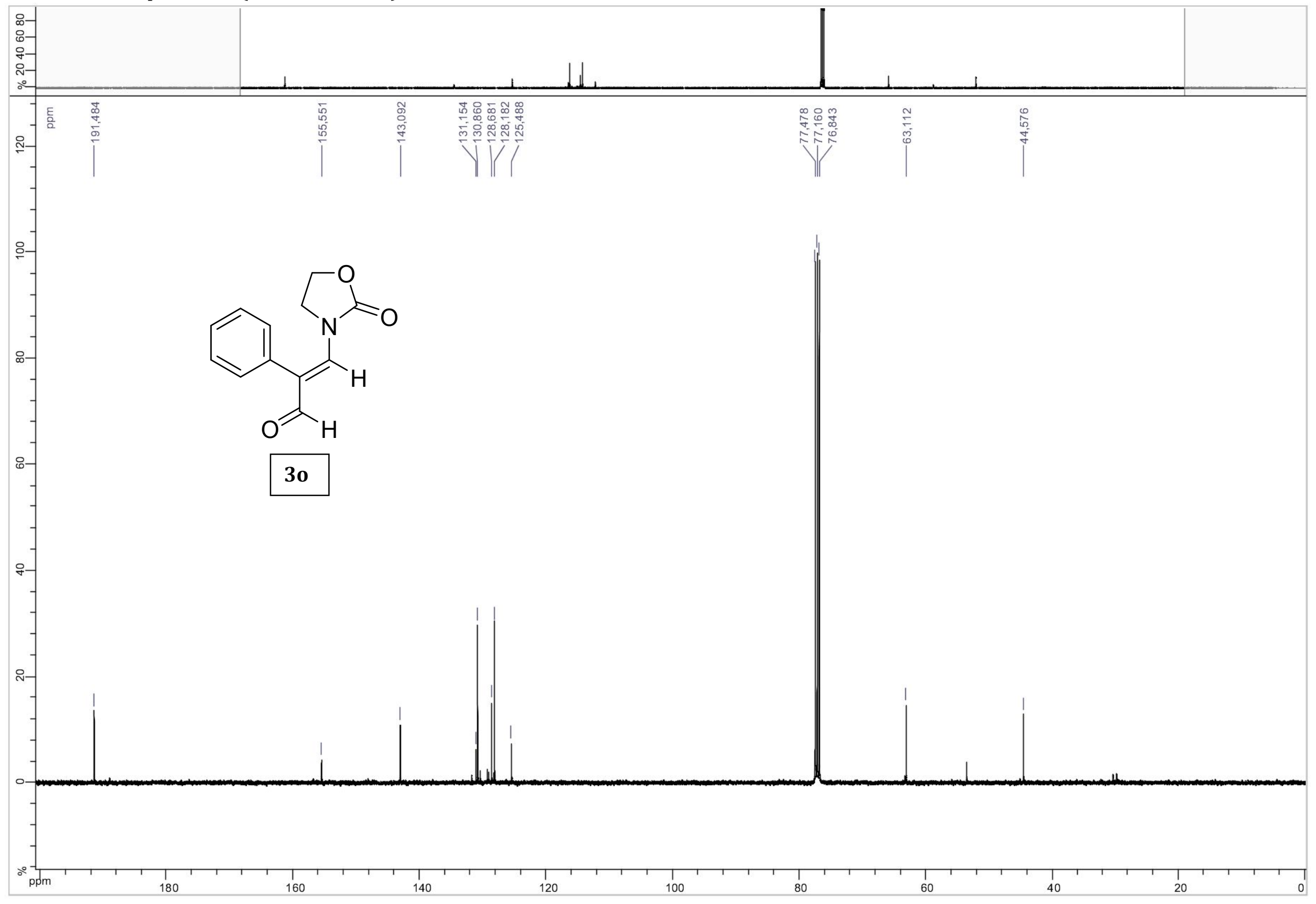


${ }^{1} \mathrm{H}$ NMR of compound $\mathbf{2 p}\left(400 \mathrm{MHz}, \mathrm{CDCl}_{3}\right)$ :

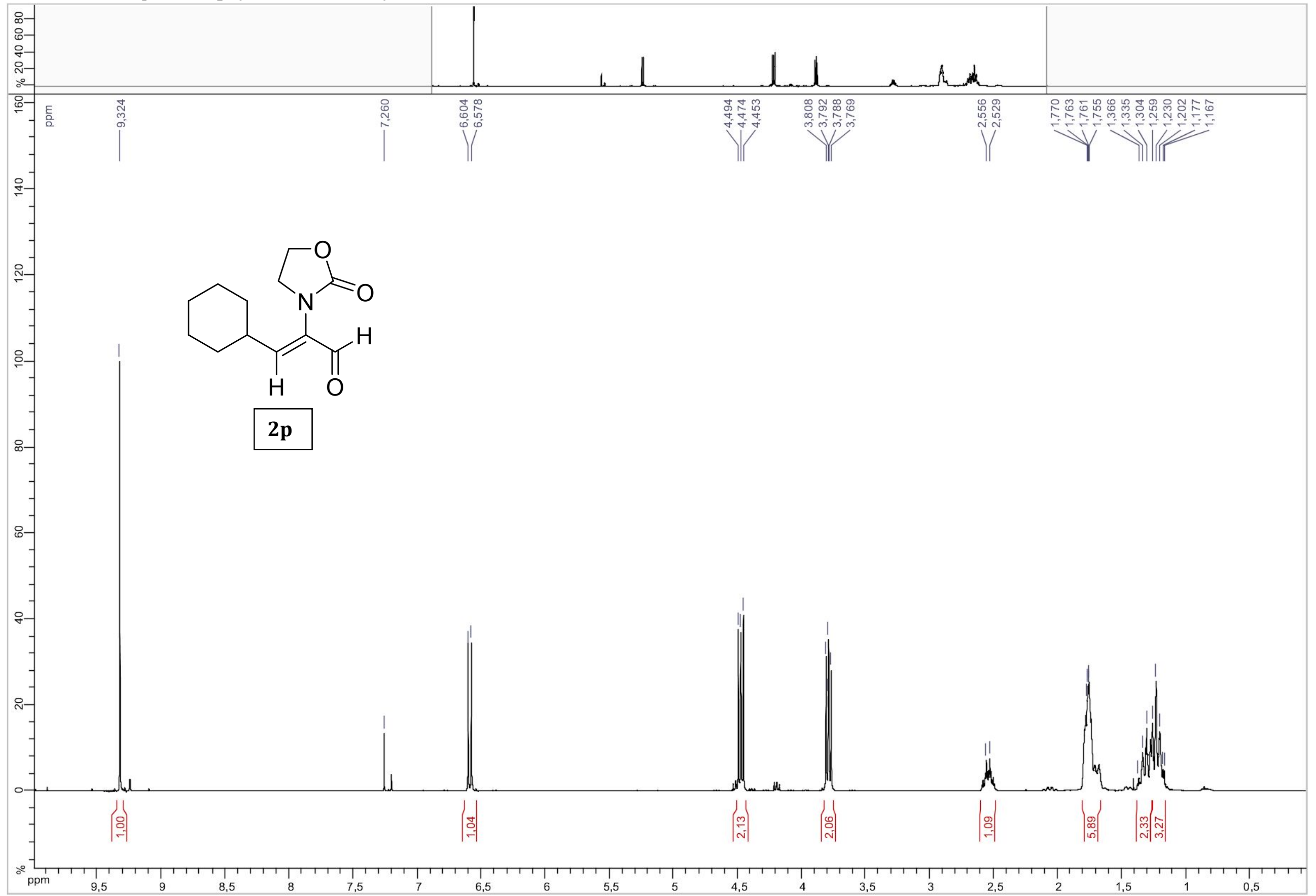


${ }^{13} \mathrm{C}$ NMR of compound $\mathbf{2 p}\left(101 \mathrm{MHz}, \mathrm{CDCl}_{3}\right)$ :

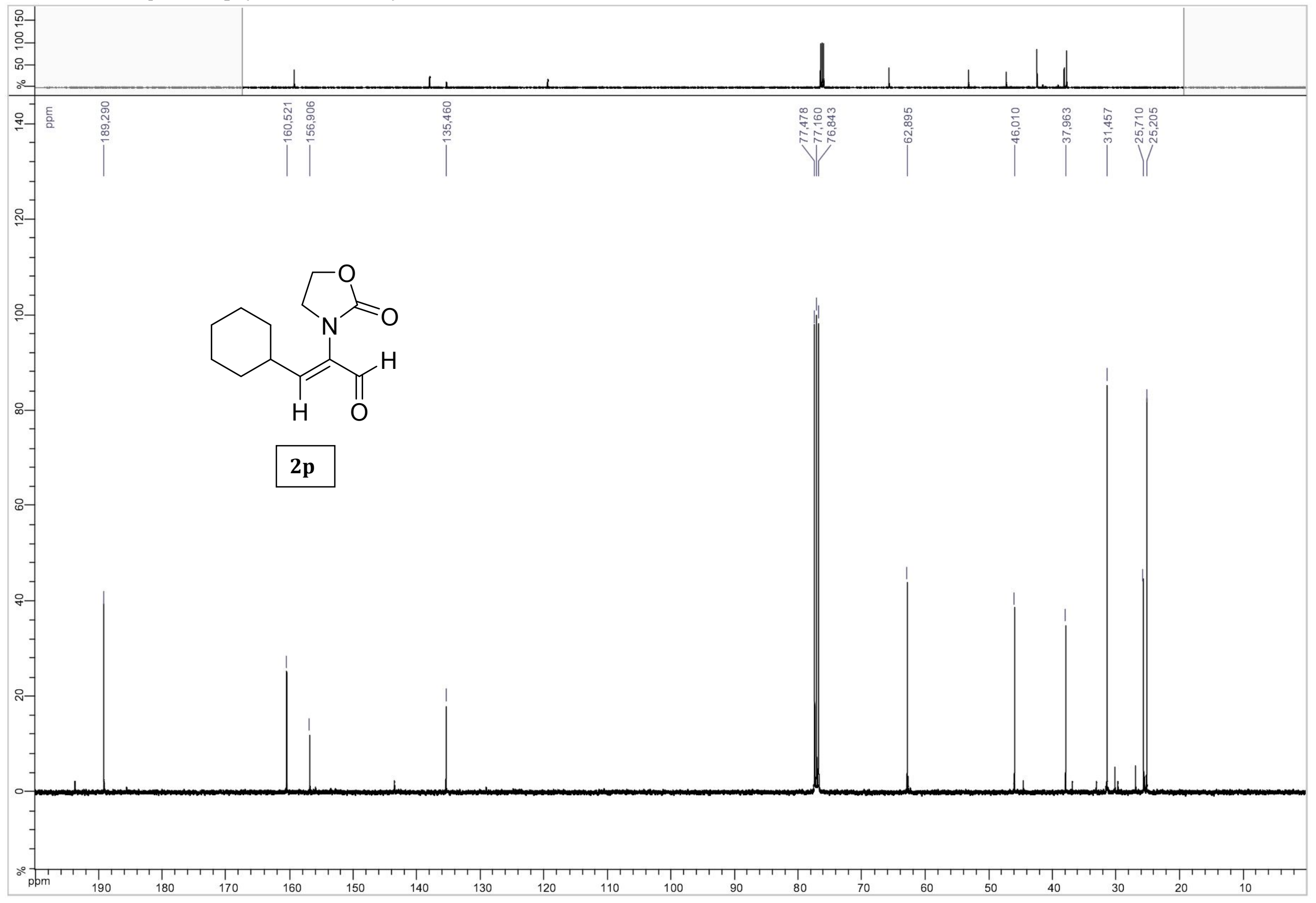


${ }^{1} \mathrm{H}$ NMR of compound 3p (400 $\mathrm{MHz}, \mathrm{CDCl}_{3}$ ):

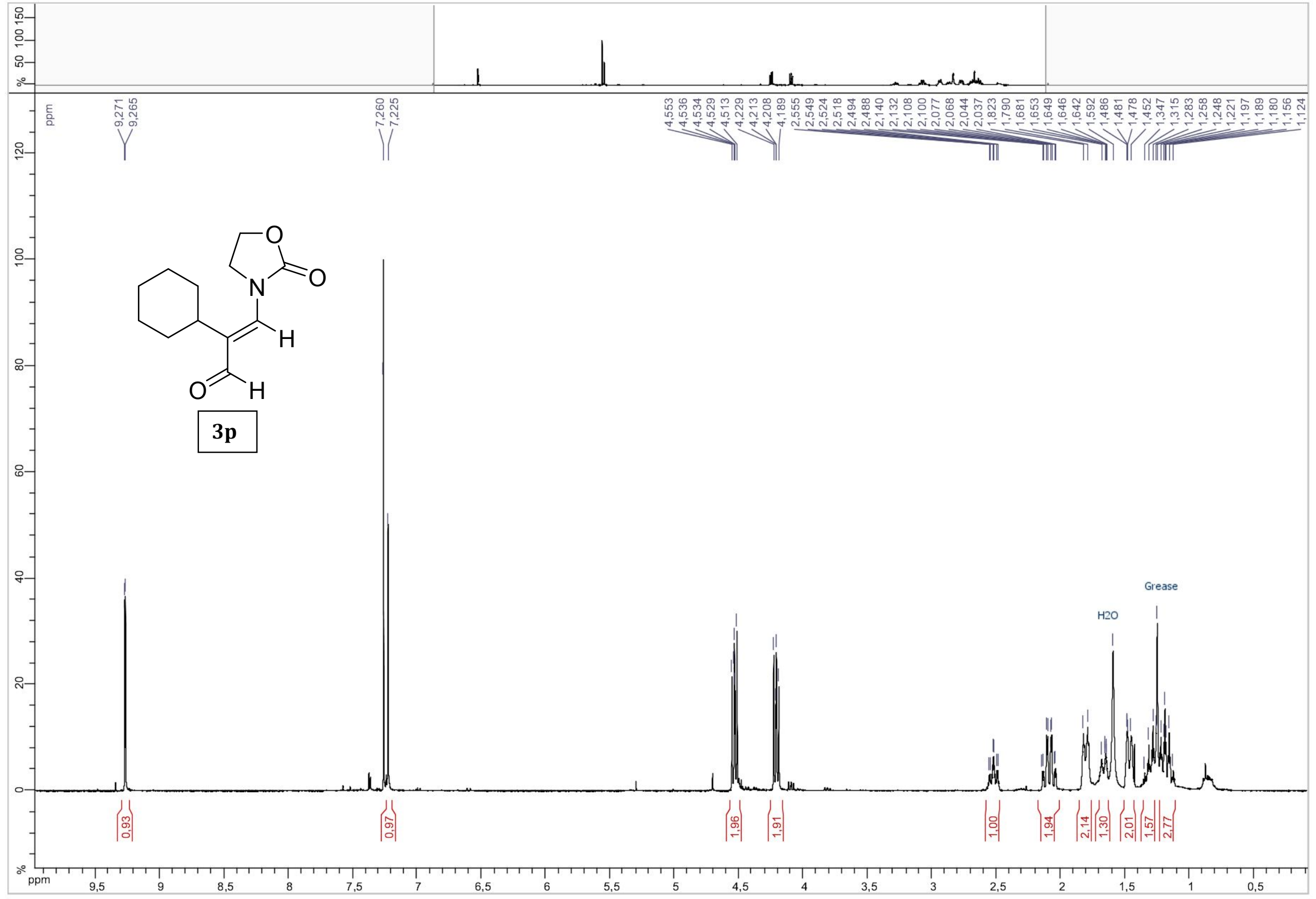


${ }^{13} \mathrm{C}$ NMR of compound 3p (101 MHz, $\left.\mathrm{CDCl}_{3}\right)$ :

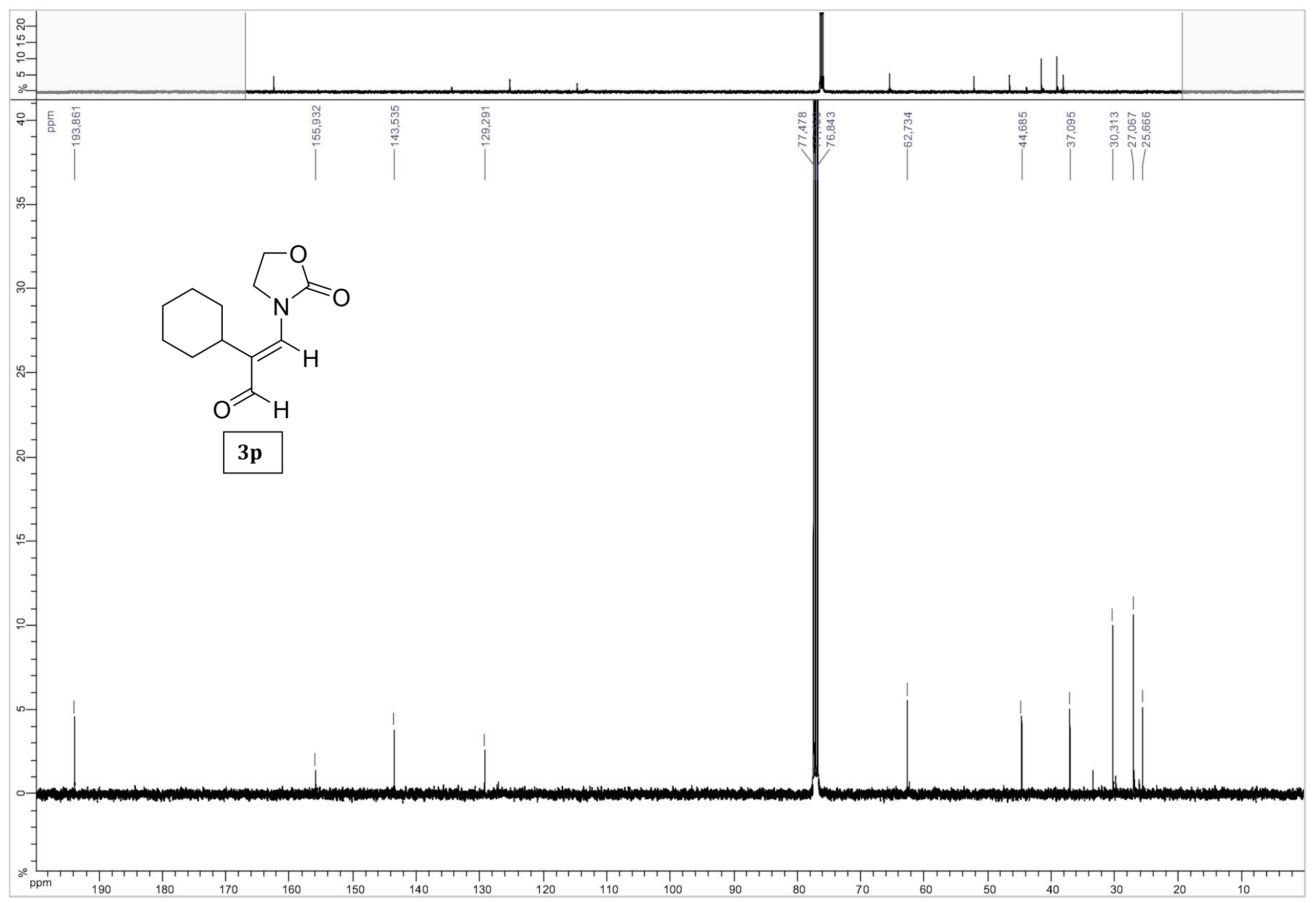


${ }^{1} \mathrm{H}$ NMR of compound 2q (400 MHz, $\left.\mathrm{CDCl}_{3}\right)$ :

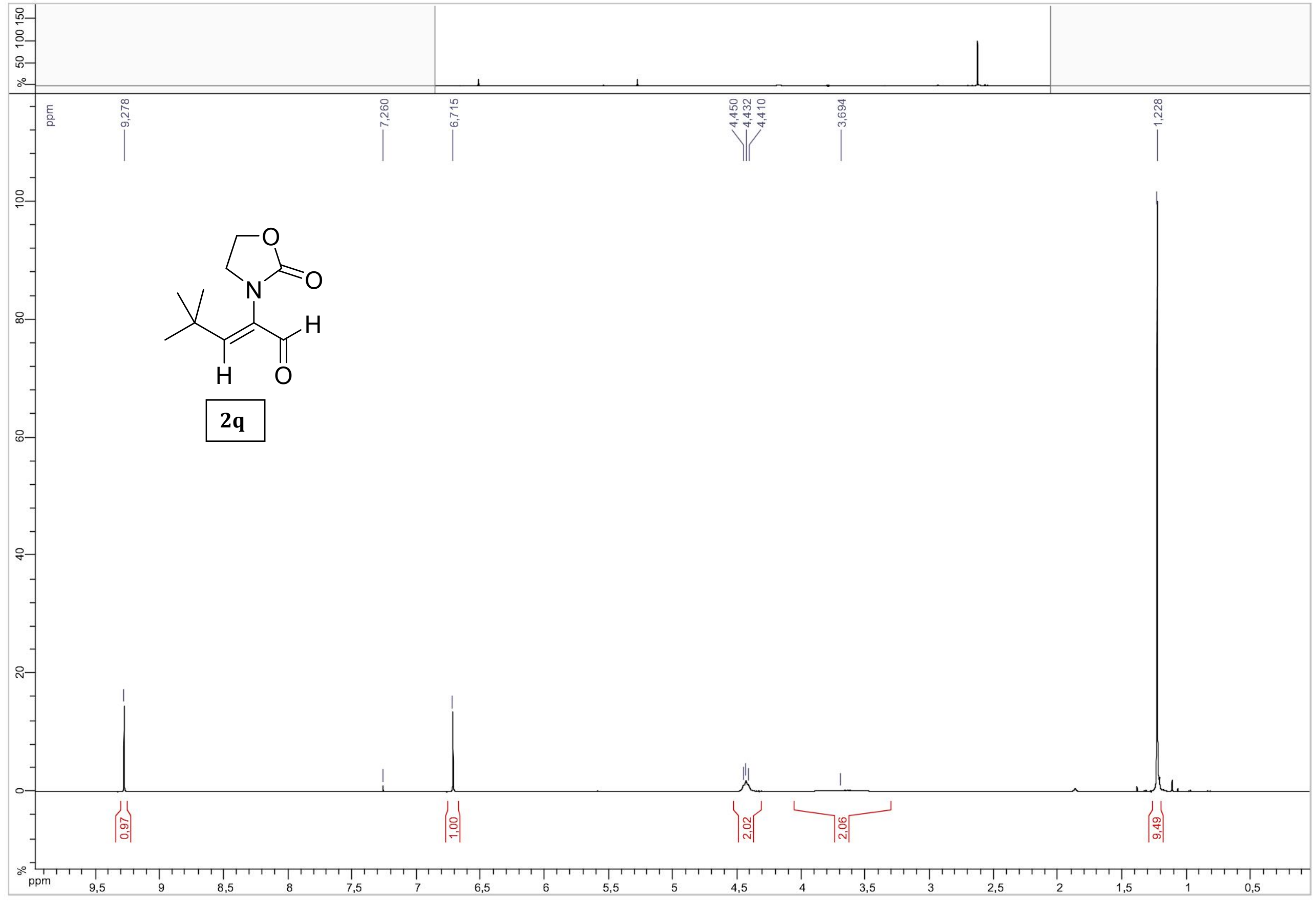


${ }^{13} \mathrm{C}$ NMR of compound 2q $\left(101 \mathrm{MHz}, \mathrm{CDCl}_{3}\right)$ :

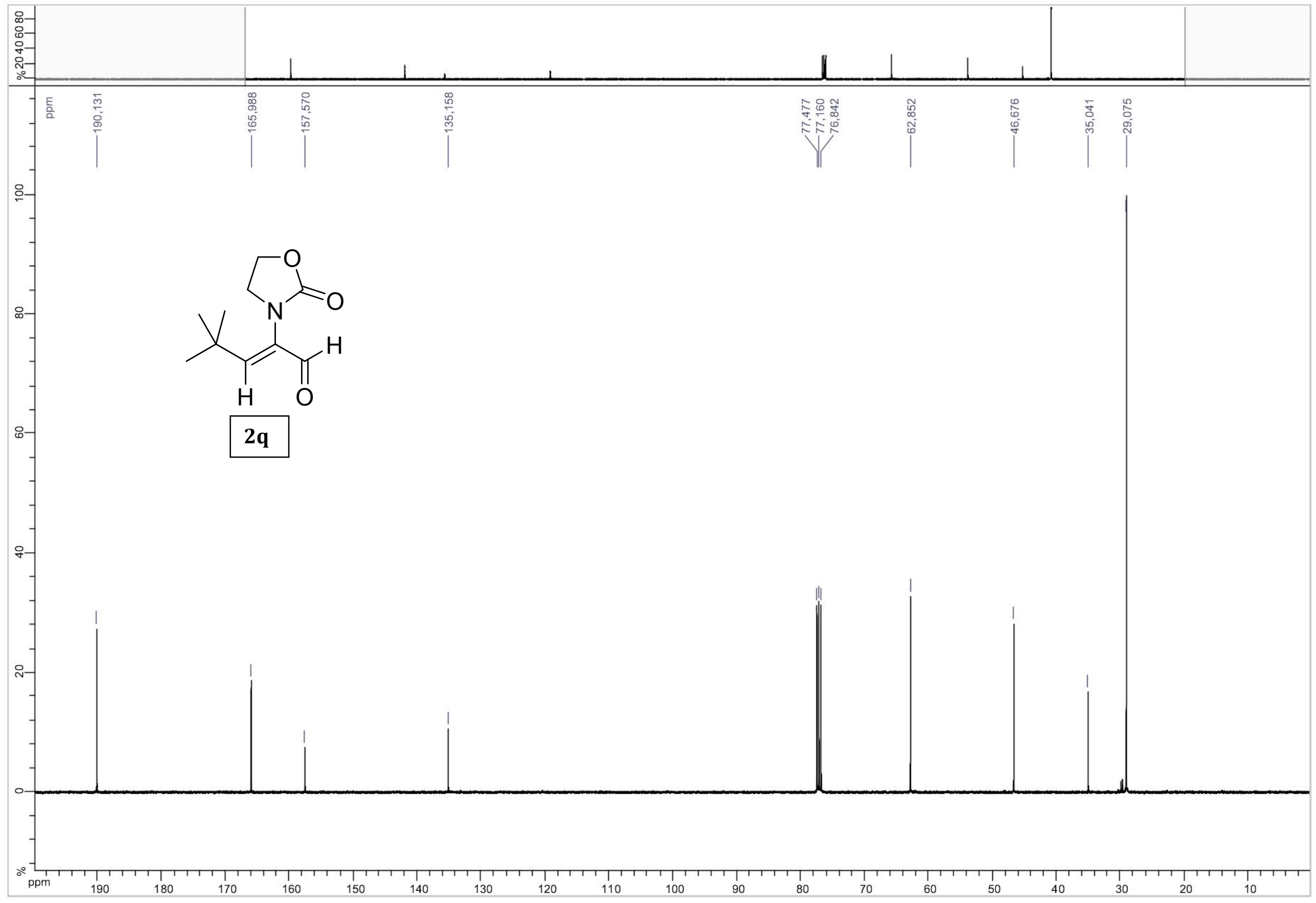


NOESY of compound 2q (400 MHz, $\mathrm{CDCl}_{3}$ ):

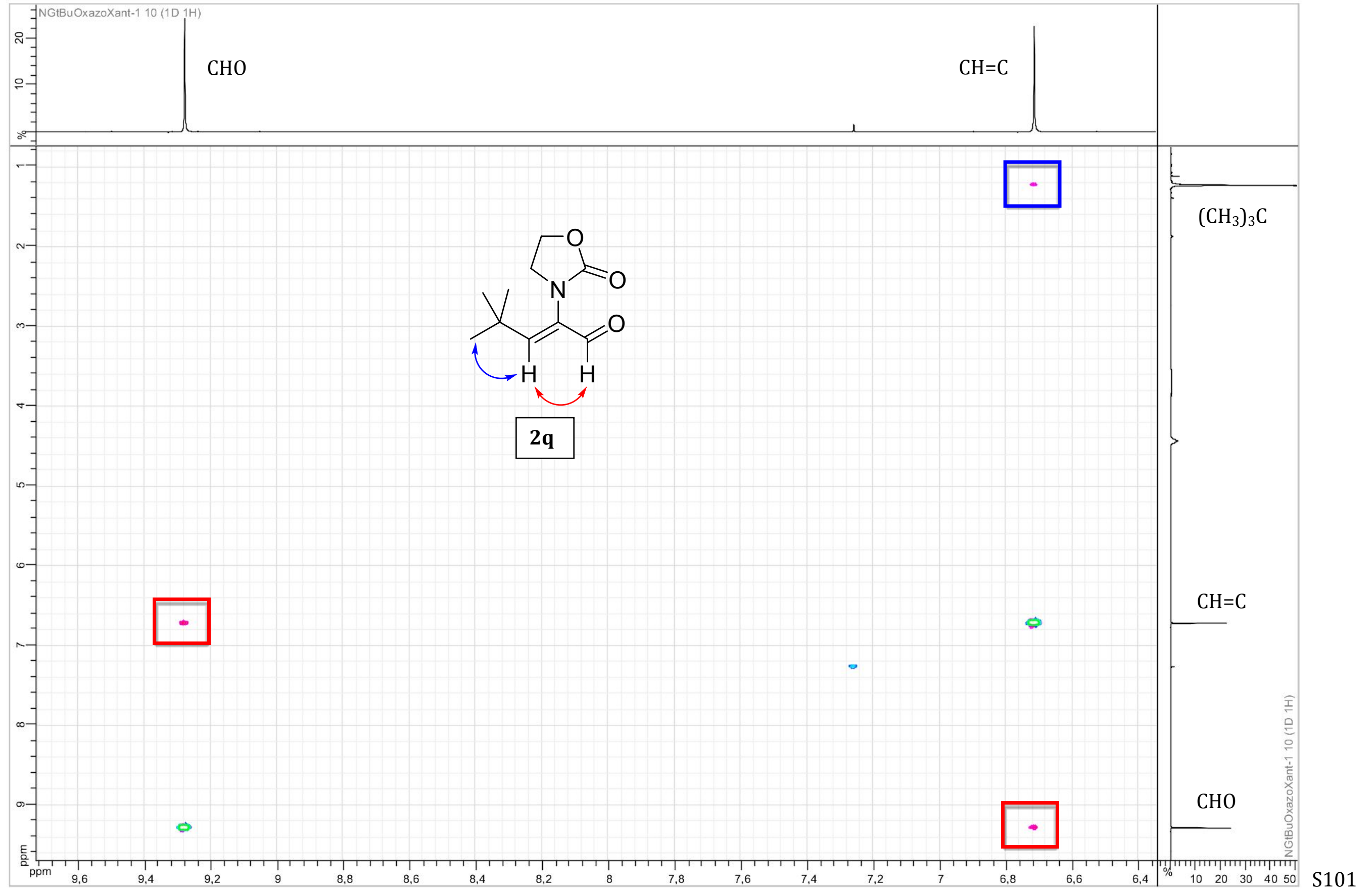

